\title{
Borehole Data Package for Wells 299-E33-334 and 299-E33-335 at Single-Shell Tank Waste Management Area B-BX-BY
}

D. G. Horton

May 2000

Prepared for

the U.S. Department of Energy

under Contract DE-AC06-76RLO 1830

Pacific Northwest National Laboratory

Richland, Washington 99352 
. . 


\section{DISCLAIMER}

This report was prepared as an account of work sponsored by an agency of the United States Government. Neither the United States Government nor any agency thereof, nor any of their employees, make any warranty, express or implied, or assumes any legal liability or responsibility for the accuracy, completeness, or usefulness of any information, apparatus, product, or process disclosed, or represents that its use would not infringe privately owned rights. Reference herein to any specific commercial product, process, or service by trade name, trademark, manufacturer, or otherwise does not necessarily constitute or imply its endorsement, recommendation, or favoring by the United States Government or any agency thereof. The views and opinions of authors expressed herein do not necessarily state or reflect those of the United States Government or any agency thereof. 


\section{DISCLAIMER}

Portions of this document may be illegible in electronic image products. Images are produced from the best available original document. 


\section{Contents}

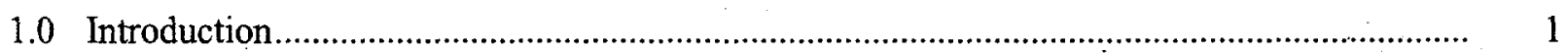

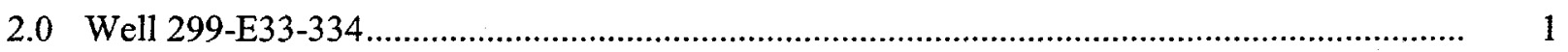

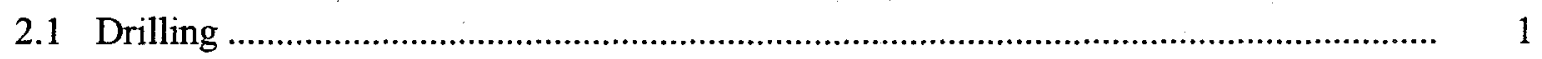

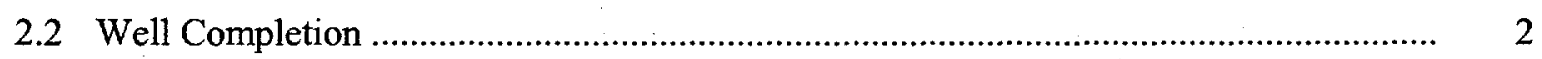

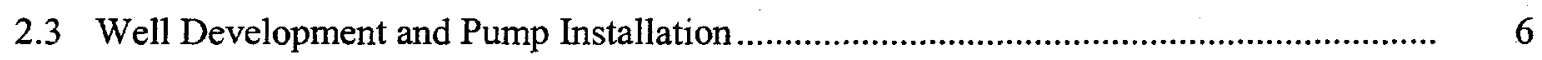

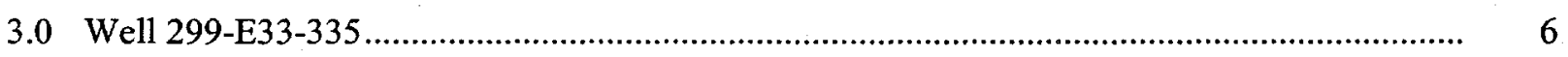

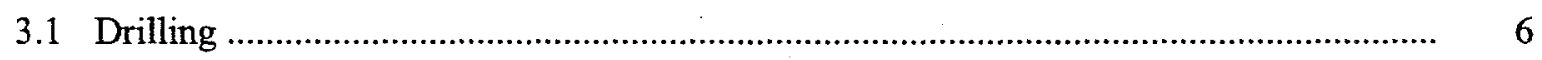

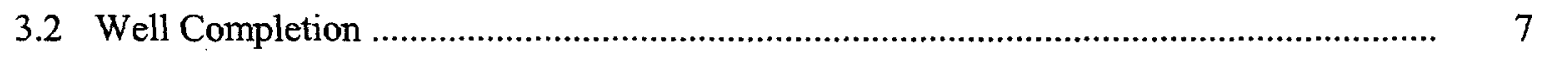

3.3 Well Development and Pump Installation ....................................................... 9

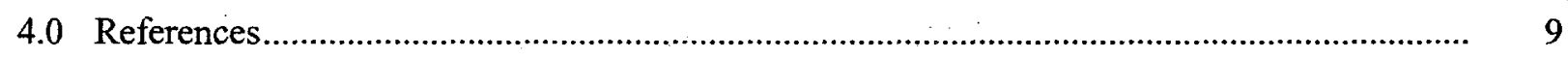

Appendix A - Well Construction and Completion Documentation ......................................... A.1

Appendix B - Moisture Content for Samples from Well 299-E33-334 .......................................... B.1

Appendix C - Borehole Geophysical Logs......................................................................... C.1

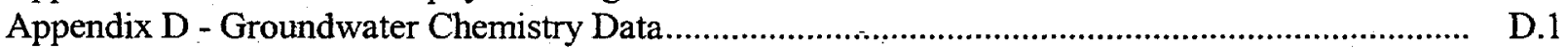

\section{Figures}

1 Map of WMA B-BX-BY and Locations of Wells in the Groundwater Monitoring Network... 3

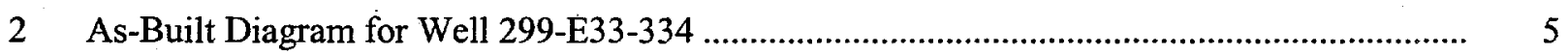

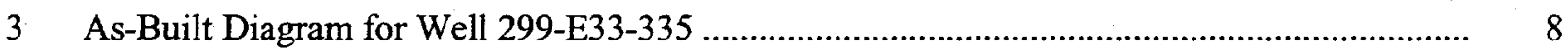

\section{Table}

1 Survey Data for New Wells at WMA B-BX-BY 


\subsection{Introduction}

Two new Resource Conservation and Recovery Act (RCRA) groundwater monitoring wells were installed at the single-shell tank farm Waste Management Area (WMA) B-BX-BY during December 1999 through February 2000 in fulfillment of Tri-Party Agreement (Ecology 1996) Milestone M-24-45. The wells are 299-E33-334 and 299-E33-335. These wells were installed in support of the WMA B-BX-BY assessment to track the movement of contaminant plumes that appear to be entering the WMA from the northeast. Well 299-E33-334 is located outside the southwest corner of the 241-BX tank farm and well 299-E33-335 is located south of the 241-BX tank farm. The locations of all wells in the extended monitoring network for WMA B-BX-BY are shown on Figure 1.

The new wells were constructed to the specifications and requirements described in Washington Administrative Code (WAC) 173-160 and WAC 173-303, in the assessment groundwater monitoring plan (Narbutovskih 2000), and in the description of work for well drilling and installation. ${ }^{1}$

This document compiles information on the drilling, construction, well development, pump installation, and sampling activities applicable to wells 299-E33-334 and 299-E33-335. Appendix A contains copies of the Well Summary Sheets (as-built diagrams), the Well Construction Summary Reports, and the geologist's logs. Appendix B contains results of laboratory analyses of moisture content on samples from 299-E33-334 (moisture data were not collected from well 299-E33-335). Appendix C contains borehole geophysical logs and Appendix D contains analytical results from groundwater samples obtained during well construction. Additional documentation concerning well construction is on file with Bechtel Hanford, Inc.

English units are used in this report because that is the system of units used by drillers to measure and

report depths and well construction details. Conversion to metric is made by multiplying feet by 0.3048 to obtain meters or multiplying inches by 2.54 to obtain centimeters.

\subsection{Well 299-E33-334}

\subsection{Drilling}

Well 299-E33-334 was drilled using a cable tool rig and drive barrel from 0 to $272 \mathrm{ft}$ below ground surface (bgs) and a cable tool rig and hard tool from $272 \mathrm{ft}$ to the total depth of $285 \mathrm{ft}$ bgs during December 1999. The well was started with temporary 11 3/4-in.-outside-diameter, carbon steel casing

\footnotetext{
${ }^{1}$ Letter from R. M. Smith, Pacific Northwest National Laboratory, Richland, Washington, to G. C. Henckel, Bechtel Hanford, Inc., dated May 26, 1999, "Description of Work for Drilling of CY 1999 RCRA Groundwater Monitoring Wells."
} 
from 0 to $51.5 \mathrm{ft}$ bgs followed by 8 5/8-in.-outside-diameter, carbon steel casing from $51.5 \mathrm{ft}$ to total depth. Approximately 800 gal of water were added to the borehole below $270 \mathrm{ft}$ to facilitate drilling. At about $207 \mathrm{ft}$ bgs, 1 gal was added to keep sediment in the drive barrel.

Sediments encountered during drilling were Hanford formation sandy gravel and gravelly sand from 0 to $47 \mathrm{ft}$ depth, Hanford formation sand with minor silty sand from 47 to $222 \mathrm{ft}$ depth, and undifferentiated Hanford formation/Plio-Pleistocene sandy gravel and silty sandy gravel from 222 to $280 \mathrm{ft}$ depth. Basalt was encountered at $280 \mathrm{ft}$ to total depth. The water table was measured at $263.85 \mathrm{ft}$ bgs on December 22 , 1999. The geologist's log is included in Appendix A.

Grab samples were collected at about $5 \mathrm{ft}$ intervals throughout the borehole for geologic description and archive. Separate samples were collected at the same intervals for analysis of moisture content. The results of the moisture analyses are in Appendix B. In addition, split spoon samples were collected between 265.5 and $268 \mathrm{ft}$ ( $80 \%$ recovery) and between 275 and $277.5 \mathrm{ft}$ (100\% recovery) depths for potential, future analysis of hydraulic conductivity and particle size distribution. The borehole and drill cuttings were monitored regularly for organic vapors and radionuclide contaminants. No contamination was detected.

The well was geophysically logged through the temporary casing using NaI spectral gamma-ray and neutron-neutron moisture instrumentation in December 1999. No man-made radionuclides were noted. The geophysical logs are in Appendix C.

\subsection{Well Completion}

The permanent casing and screen were installed in well 299-E33-334 in December 1999. A 4-in.inner-diameter, stainless steel, continuous wire-wrap ( 0.02 -in. slot) screen was set from 282.72 to $257.70 \mathrm{ft}$ bgs. The permanent casing is 4 -in.-inner-diameter, stainless steel from $257.7 \mathrm{ft}$ bgs to $2.5 \mathrm{ft}$ above ground surface. The bottom of the screen has a 4-in. stainless steel end cap.

The filter pack is 10 to 20 mesh silica sand from 284.1 to $247.4 \mathrm{ft}$ bgs. The annular seal is 8 mesh bentonite crumbles from 247.4 to $10.5 \mathrm{ft}$ bgs and Portland cement with bentonite from $10.5 \mathrm{ft}$ to the surface. A $4 \mathrm{ft}$ by $4 \mathrm{ft}$ by 6 in. concrete pad was placed around the well at the surface. A protective casing with locking cap, four protective steel posts, and a brass marker stamped with the well number were set into the concrete pad. The as-built diagram for well 299-E33-334 is shown in Figure 2. The Well Summary Sheet and the Well Construction Summary Report are in Appendix A.

The vertical and horizontal coordinates of the well were surveyed in March 2000. The horizontal position of the well was determined by Global Positioning System observations referenced to horizontal control stations established by Rogers Surveying, Inc., Richland, Washington. The coordinates are Washington Coordinate System, South Zone, NAD83(91) datum. Vertical datum is NAVD 1988 and is based on existing bench marks established by the U.S. Corps of Engineers. Survey data are included in Table 1. 


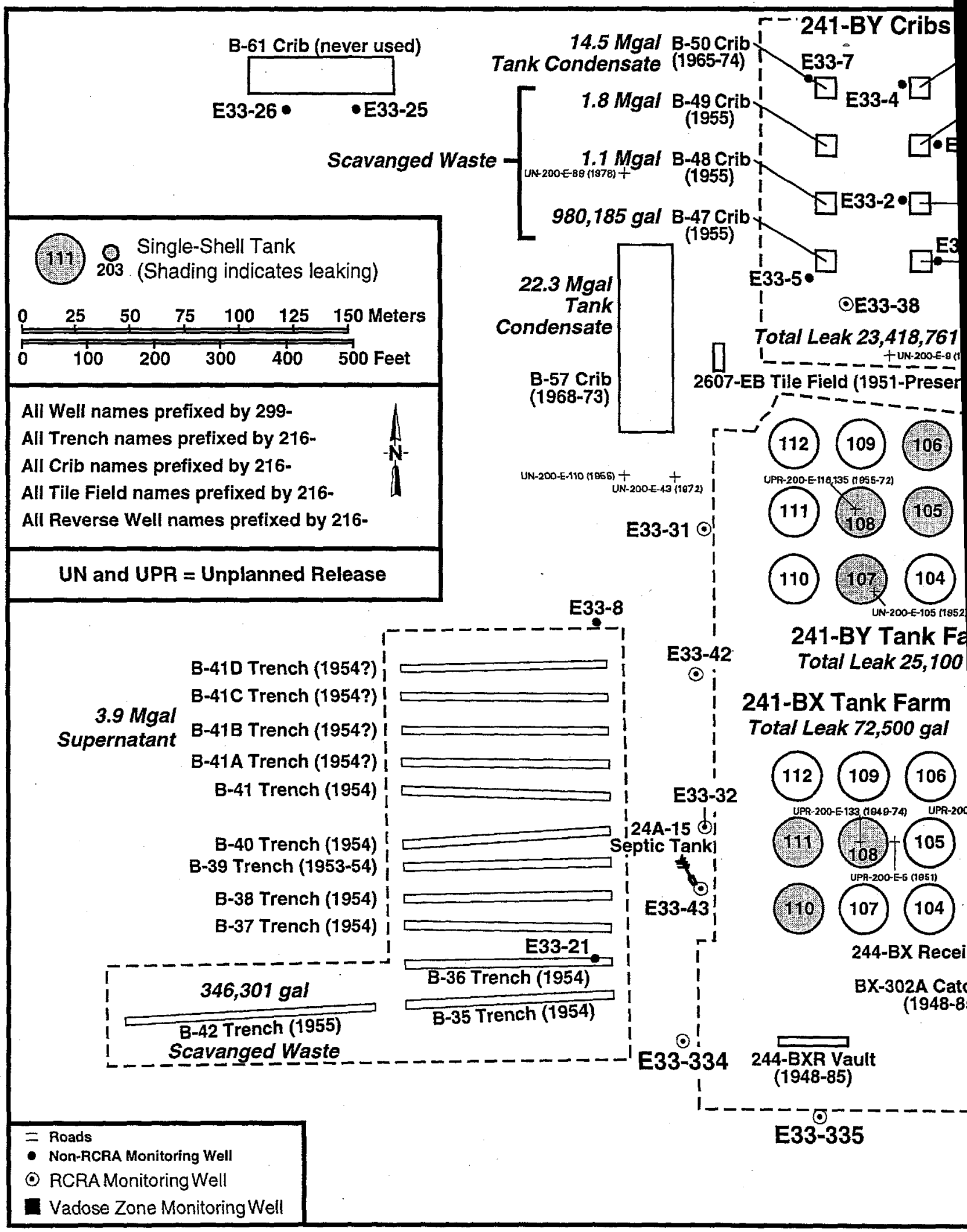

Figure 1. Map of WMA B-BX-BY and Local 


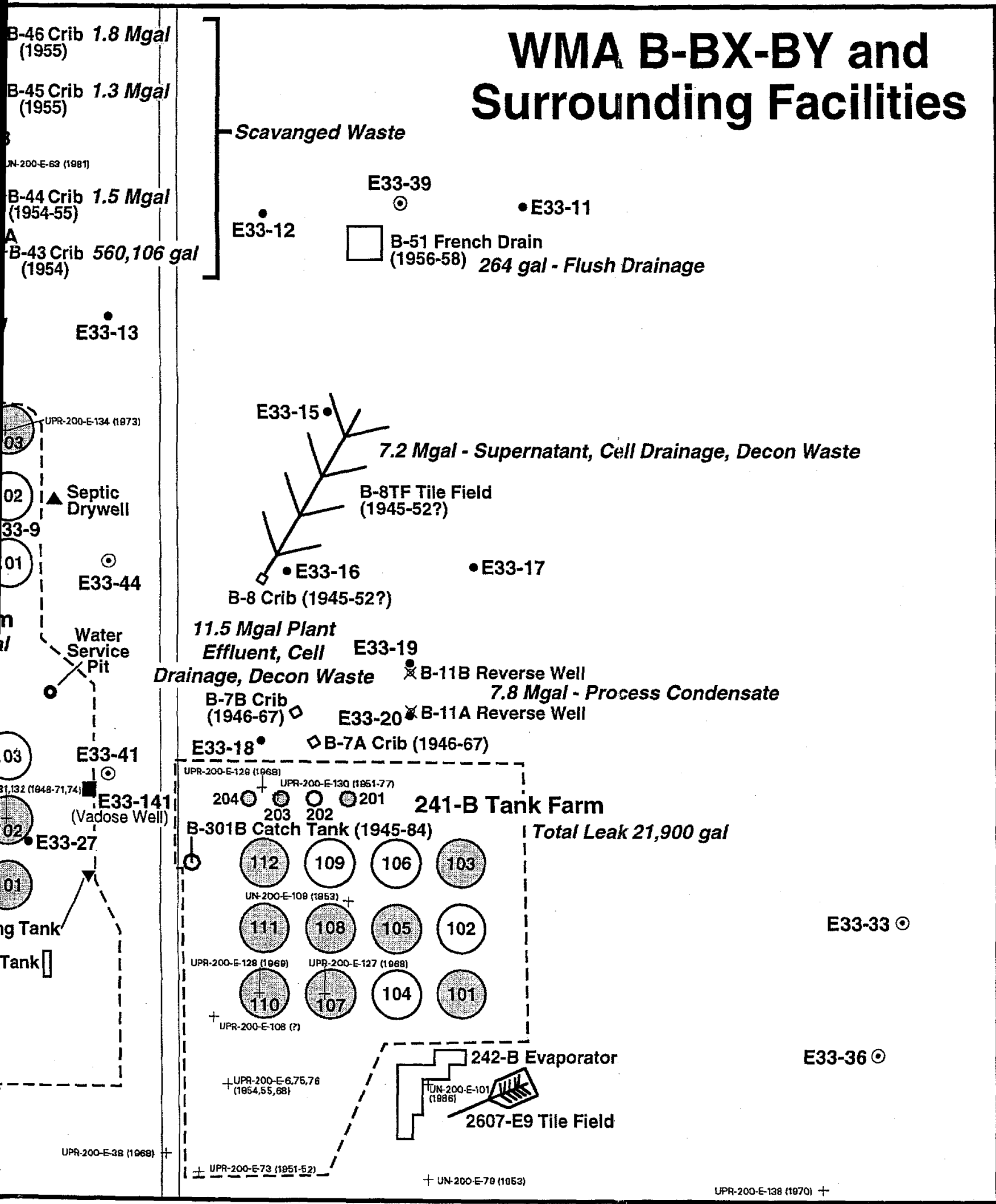

2000/DCL/B-BX-BY/007

hs of Wells in the Groundwater Monitoring Network 


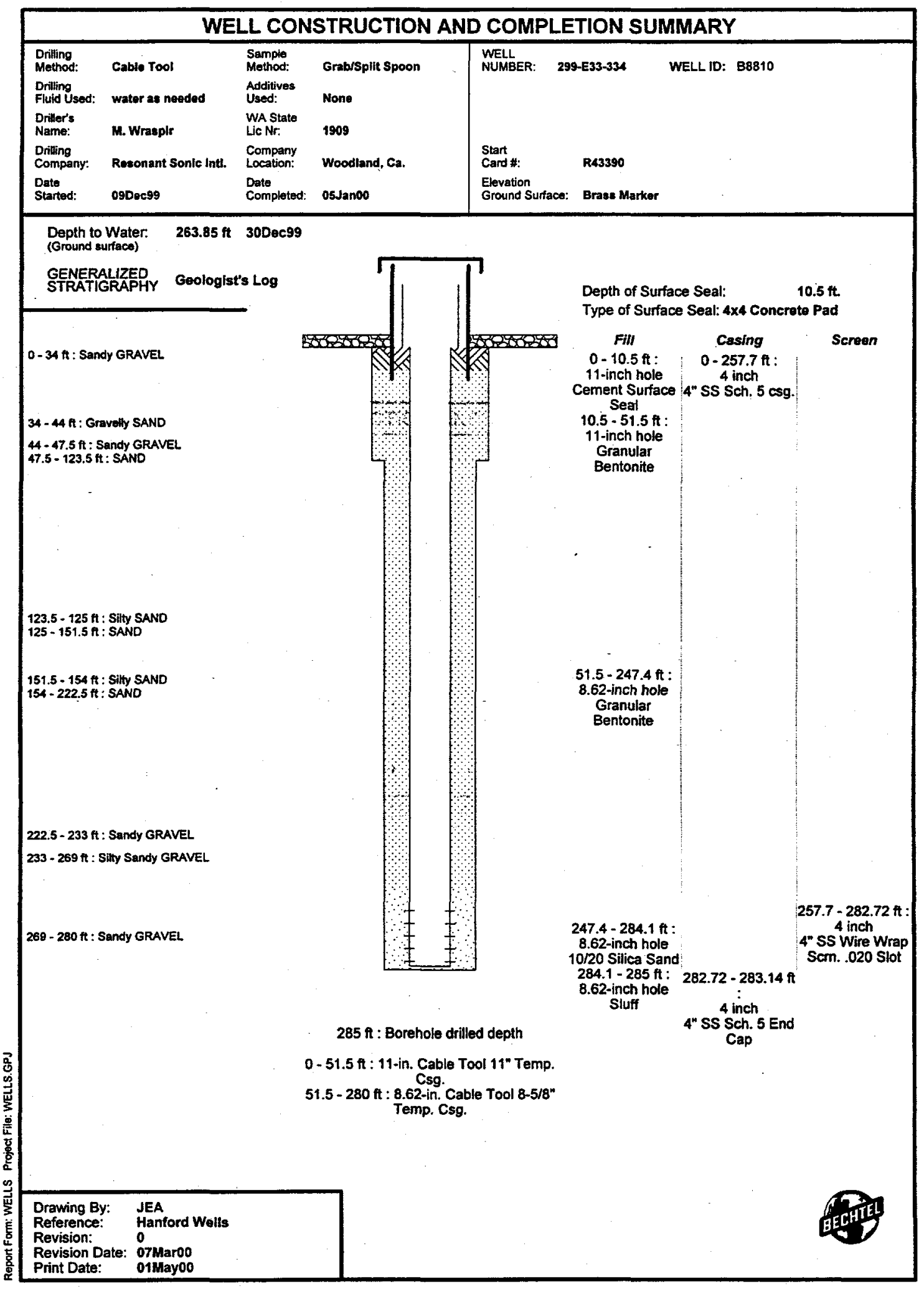

Figure 2. As-Built Diagram for Well 299-E33-334. Elevation reference point is ground surface. 
Table 1. Survey Data for New Wells at WMA B-BX-BY

\begin{tabular}{|c|c|c|c|c|}
\hline Well Name & $\begin{array}{c}\text { Easting } \\
\text { m (ft) }\end{array}$ & $\begin{array}{l}\text { Northing } \\
\text { m (ft) }\end{array}$ & $\begin{array}{c}\text { Elevation } \\
\text { m (ft) }\end{array}$ & \\
\hline \multirow[t]{3}{*}{ 299-E33-334 } & $\begin{array}{c}573,514,716 \\
(1,881,604.286) \\
\end{array}$ & $\begin{array}{c}137,256.371 \\
(450,314.820) \\
\end{array}$ & & Center of Casing \\
\hline & & & $\begin{array}{c}204.207 \\
(670.003) \\
\end{array}$ & "X" on Casing \\
\hline & $\begin{array}{c}573,514.698 \\
(1,881,604.227)\end{array}$ & $\begin{array}{c}137,256.718 \\
(450,315.958)\end{array}$ & $\begin{array}{c}203.288 \\
(699.953)\end{array}$ & Brass Cap \\
\hline \multirow[t]{3}{*}{ 299-E33-335 } & $\begin{array}{c}573,568.442 \\
(1,881,780.552)\end{array}$ & $\begin{array}{c}137,222.229 \\
(450,202.806)\end{array}$ & & Center of Casing \\
\hline & & & $\begin{array}{c}204.262 \\
(670.183)\end{array}$ & "X" on Casing \\
\hline & $\begin{array}{c}573,568.486 \\
(1,881,780.696)\end{array}$ & $\begin{array}{c}137,222.575 \\
(450,203.941)\end{array}$ & $\begin{array}{c}203.415 \\
(667.370)\end{array}$ & Brass Cap \\
\hline
\end{tabular}

\subsection{Well Development and Pump Installation}

Well 299-E33-334 was developed in January 2000. A temporary, $3 \mathrm{hp}$, submersible pump was used to remove approximately $2,370 \mathrm{gal}$ of formation water from the well at $23 \mathrm{gal} / \mathrm{min}$. The pump intake was $279.17 \mathrm{ft}$ bgs or at about $11.9 \mathrm{ft}$ below the water table. The final turbidity was 0.90 NTU. No drawdown was observed during the test. A groundwater sample was collected from the well at $279.17 \mathrm{ft}$ bgs after well development. The results of the laboratory analysis of the sample are in Appendix D.

A dedicated Hydrostar sampling pump was installed in well 299-E33-334 in January 2000. The sampling pump intake is at $273.47 \mathrm{ft}$ bgs or about $9.6 \mathrm{ft}$ below the water table. Static water level was $263.85 \mathrm{ft}$ bgs on December 30, 1999.

\subsection{Well 299-E33-335}

\subsection{Drilling}

The sonic drilling method was used to begin well 299-E33-335 in December 1999. Temporary worker, $113 / 4$-in.-outside-diameter, carbon steel casing was placed from the surface to $50.4 \mathrm{ft}$ bgs. An air rotary drill rig was used to finish the drilling and placed temporary $85 / 8$-in.-outside-diameter, carbon steel casing from $50 \mathrm{ft}$ to $286 \mathrm{ft}$ bgs. No water was added to the borehole during drilling; however, about 800 gal were added during completion activities to control hydraulic head. 
Sediments encountered during drilling were Hanford formation sandy gravel and silty sandy gravel from the surface to about $58 \mathrm{ft}$ depth; Hanford formation sand from $58 \mathrm{ft}$ to about $226 \mathrm{ft}$ depth; and undifferentiated Hanford formation/Plio-Pleistocene silty sandy gravel and sandy gravel from $226 \mathrm{ft}$ to $280.5 \mathrm{ft}$ depth. Basalt was encountered at $280.5 \mathrm{ft}$ to total depth ( $286 \mathrm{ft} \mathrm{bgs}$ ). Water level was measured at $264.40 \mathrm{ft}$ bgs on February 9, 2000. The geologist's log is included in Appendix A.

Grab samples were collected for lithologic description and archive at approximately $5 \mathrm{ft}$ intervals from $55 \mathrm{ft}$ to the bottom of the borehole. In addition, split spoon samples were collected from 149 to $151.5 \mathrm{ft}, 180$ to $182.4 \mathrm{ft}$, and 209.8 to $212.2 \mathrm{ft}$ bgs for future paleomagnetic analysis to aid stratigraphic interpretation. The borehole cuttings were monitored regularly for organic vapors and radionuclide contaminants. No contamination was found.

Well 299-E33-335 was geophysically logged through the temporary casing using NaI spectral gamma-ray and neutron-neutron moisture instrumentation in February 2000. No man-made radionuclides were noted. The geophysical logs are in Appendix C.

\subsection{Well Completion}

Well 299-E33-335 was completed in February 2000. During initial completion activities, the permanent 4-in.-inner-diameter casing and screen accidentally were lifted about $10 \mathrm{ft}$ as the temporary casing was being removed. Consequently, all the stainless steel casing and screen were removed from the borehole and the screen was visually inspected for damage. None was apparent. The sand pack was drilled out of the borehole and well completion resumed.

A 4-in.-inner-diameter, stainless steel, wire wrap (0.02-in. slot) screen was set in well 299-E33-335 from 280.03 to $260.01 \mathrm{ft}$ bgs. A stainless steel end cap was put on the bottom end of the screen. Permanent, 4-in.-inner-diameter, stainless steel casing was installed from $260.01 \mathrm{ft}$ bgs to $2.2 \mathrm{ft}$ above ground surface. The total length of the well from top of the permanent casing to bottom of the endcap is 282.63 .

The filter pack consists of 10-20 mesh silica placed around the casing from 281.9 to $250.5 \mathrm{bgs}$. The annular seal is granular bentonite pellets ( 8 mesh) between $250.5 \mathrm{ft}$ and $10.9 \mathrm{ft}$ bgs. Portland cement with bentonite was placed from $10.9 \mathrm{ft}$ to the surface. About $0.7 \mathrm{ft}$ of fine-grained sediment was in the bottom of the well after well completion.

A $4 \mathrm{ft}$ by $4 \mathrm{ft}$ by 6 in. concrete pad was placed around the well at the surface. A protective casing with locking cap, four protective steel posts, and a brass marker stamped with the well number were set into the concrete. The as-built diagram for well 299-E33-335 is shown in Figure 3. The Well Summary Sheet and the Well Construction Summary Report are included in Appendix A.

The vertical and horizontal coordinates of the well were surveyed in March 2000. The horizontal position of the well was determined by Global Positioning System observations referenced to horizontal control stations established by Rogers Surveying, Inc., Richland, Washington. The coordinates are 


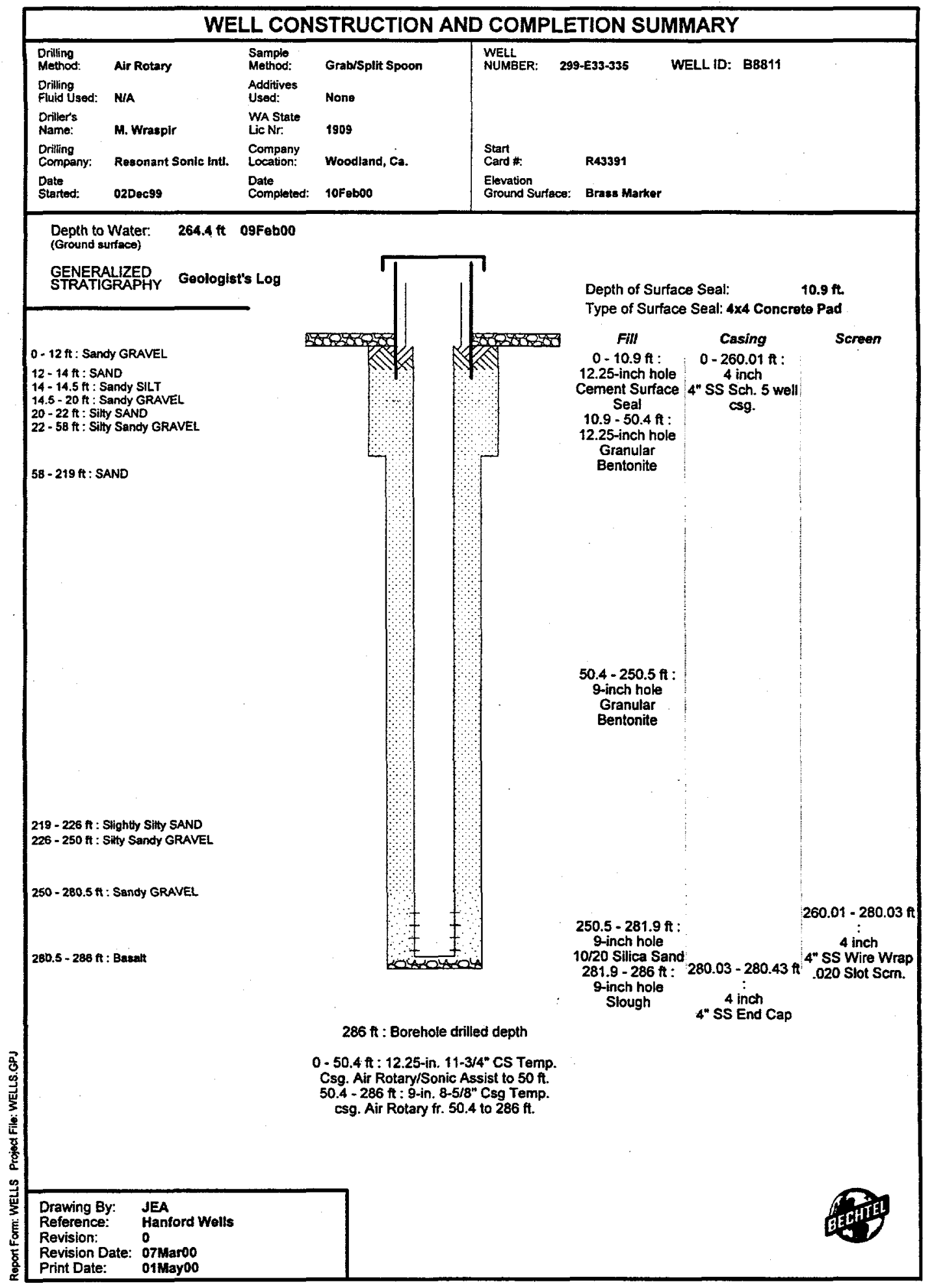

Figure 3. As-Built Diagram for Well 299-E33-335. Elevation reference point is ground surface. 
Washington Coordinate System, South Zone, NAD83(91) datum. Vertical datum is NAVD 1988 and is based on existing bench marks established by the U.S. Corps of Engineers. Survey data are included in Table 1.

\subsection{Well Development and Pump Installation}

Well 299-E33-335 was developed in February 2000. A temporary submersible pump was used to remove approximately 2280 gal of formation water from the well. Drawdown was less than $0.1 \mathrm{ft}$ at 22 to $23 \mathrm{gal} / \mathrm{min}$. The pump intake was at $272.2 \mathrm{ft}$ bgs or at about $7.6 \mathrm{ft}$ below the water table. The final turbidity was 0.74 NTU. A groundwater sample was collected after well development from $272.2 \mathrm{ft}$ bgs. Analytical results are in Appendix D.

A dedicated Hydrostar sampling pump was installed in well 299-E33-335 in February 2000. The sampling pump intake is at $270.9 \mathrm{ft}$ bgs or at about $6.5 \mathrm{ft}$ below the water table. The static water level was $264.4 \mathrm{ft}$ bgs on February 9, 2000.

\subsection{References}

Ecology - Washington State Department of Ecology, U.S. Environmental Protection Agency, and U.S. Department of Energy. 1996. Hanford Federal Facility Agreement and Consent Order. Document No. 89-10, Rev. 4 (The Tri-Party Agreement), Ecology, Olympia, Washington.

Narbutovskih, S. M. 2000. Groundwater Quality Assessment Plan for Single-Shell Waste Management Area $B-B X-B Y$ at the Hanford Site. PNNL-13022, Pacific Northwest National Laboratory, Richland, Washington.

RCRA - Resource Conservation and Recovery Act. 1976. Public Law 94-580, as amended, 90 Stat. 2795,42 USC 6901 et seq.

WAC 173-160, Washington Administrative Code. Minimum Standards for Construction and Maintenance of Wells. Olympia, Washington.

WAC 173-303, Washington Administrative Code. Dangerous Waste Regulations. Olympia, Washington. 
Appendix A

Well Construction and Completion Documentation 


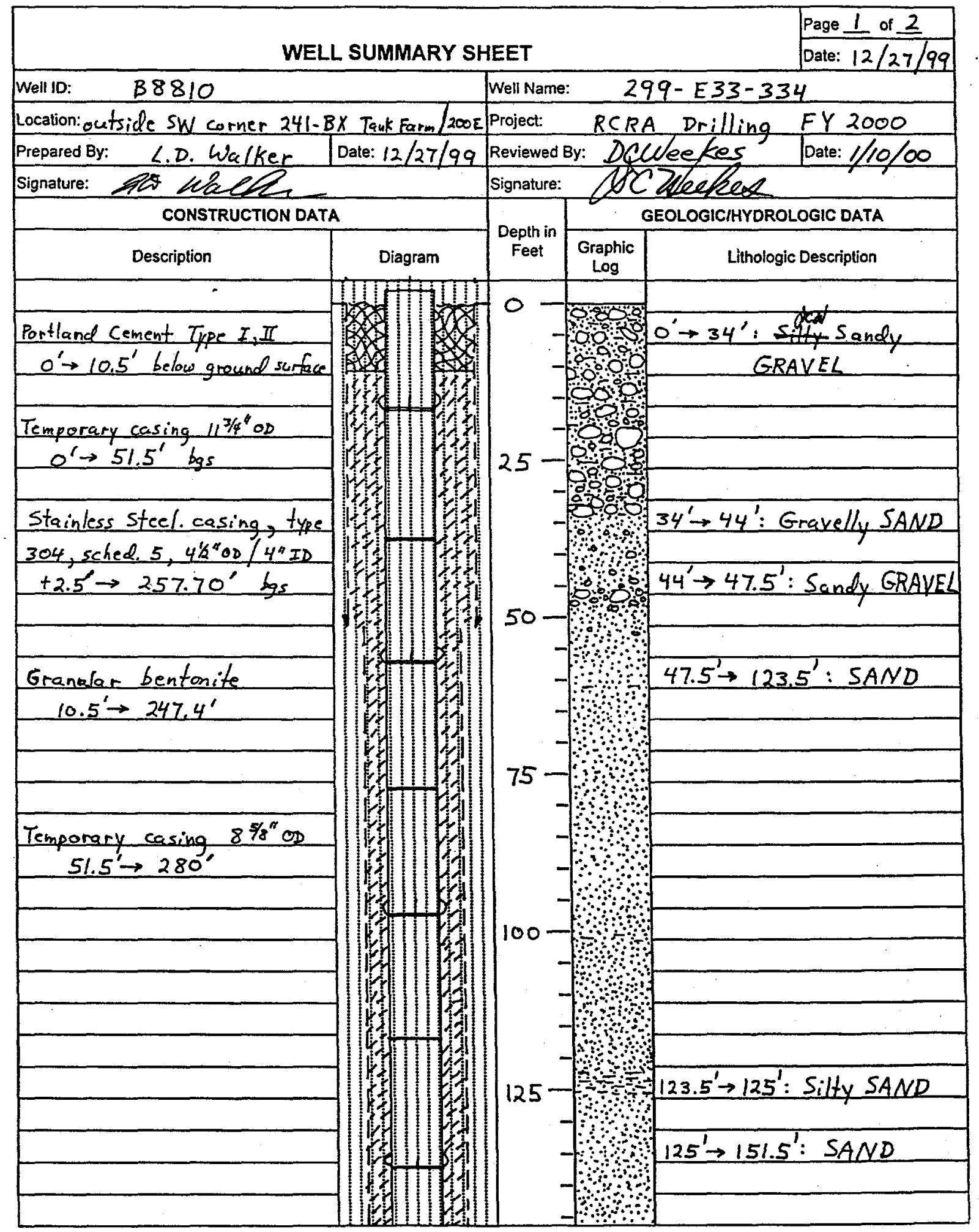




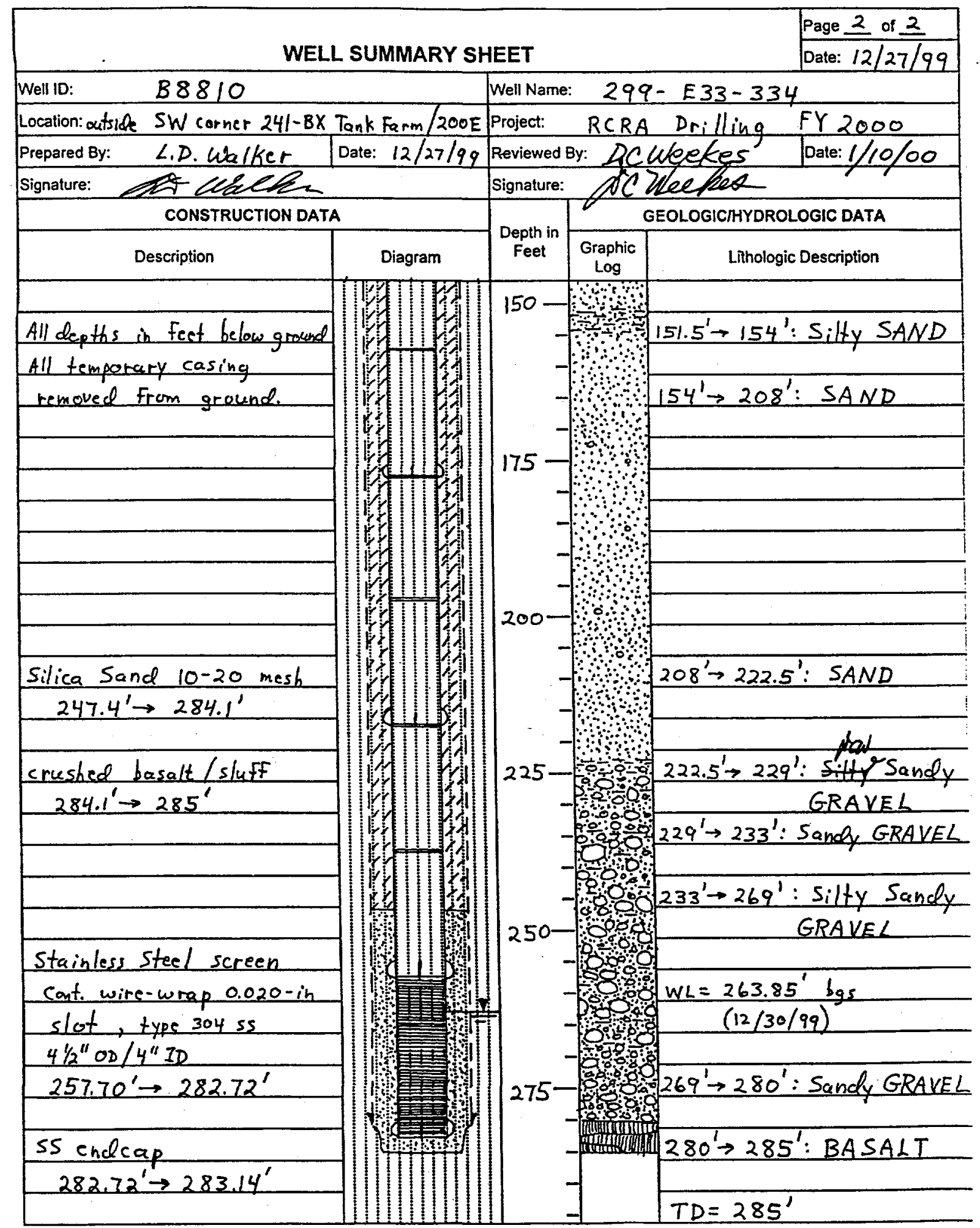

BHI-EE-189 (12/9n) 


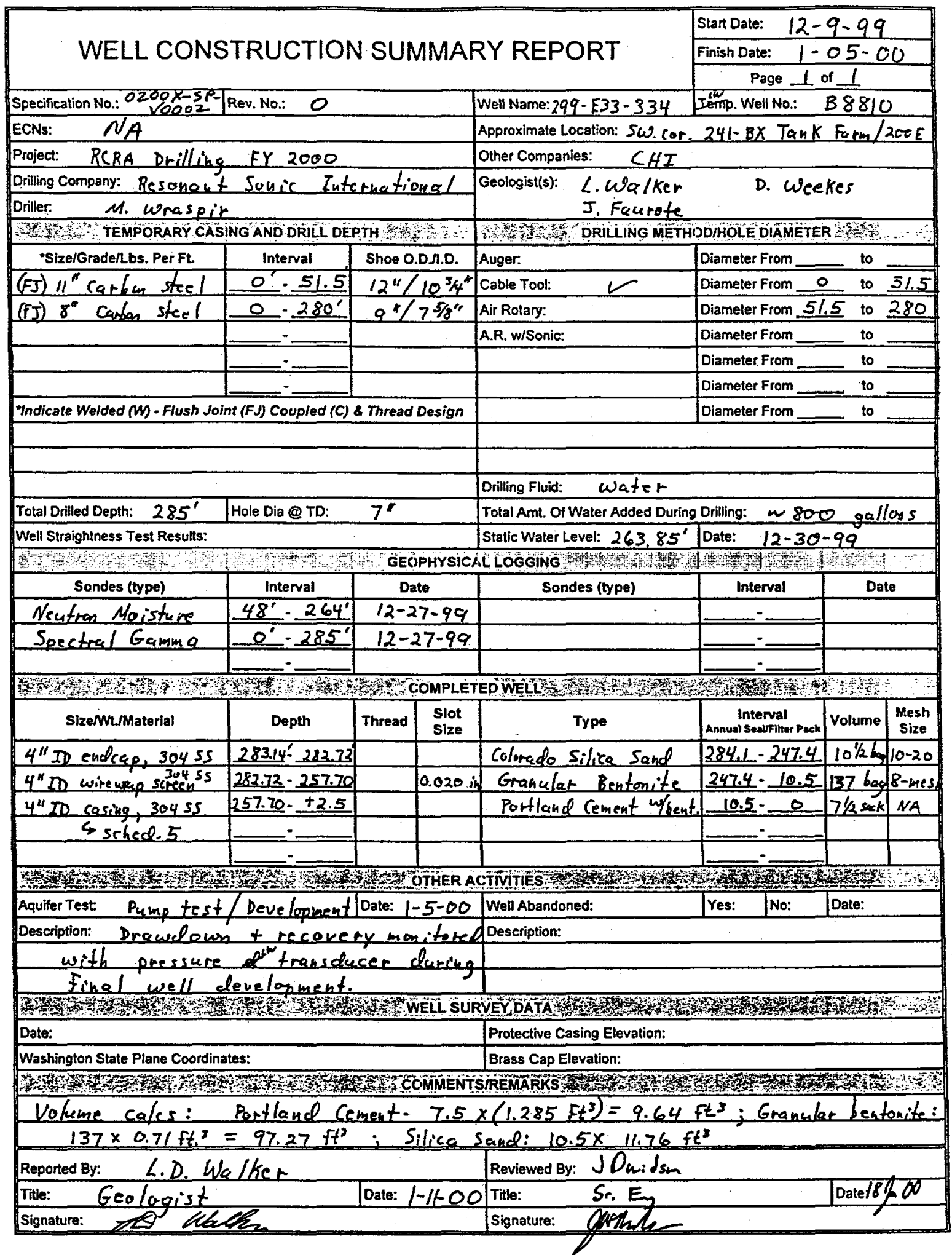




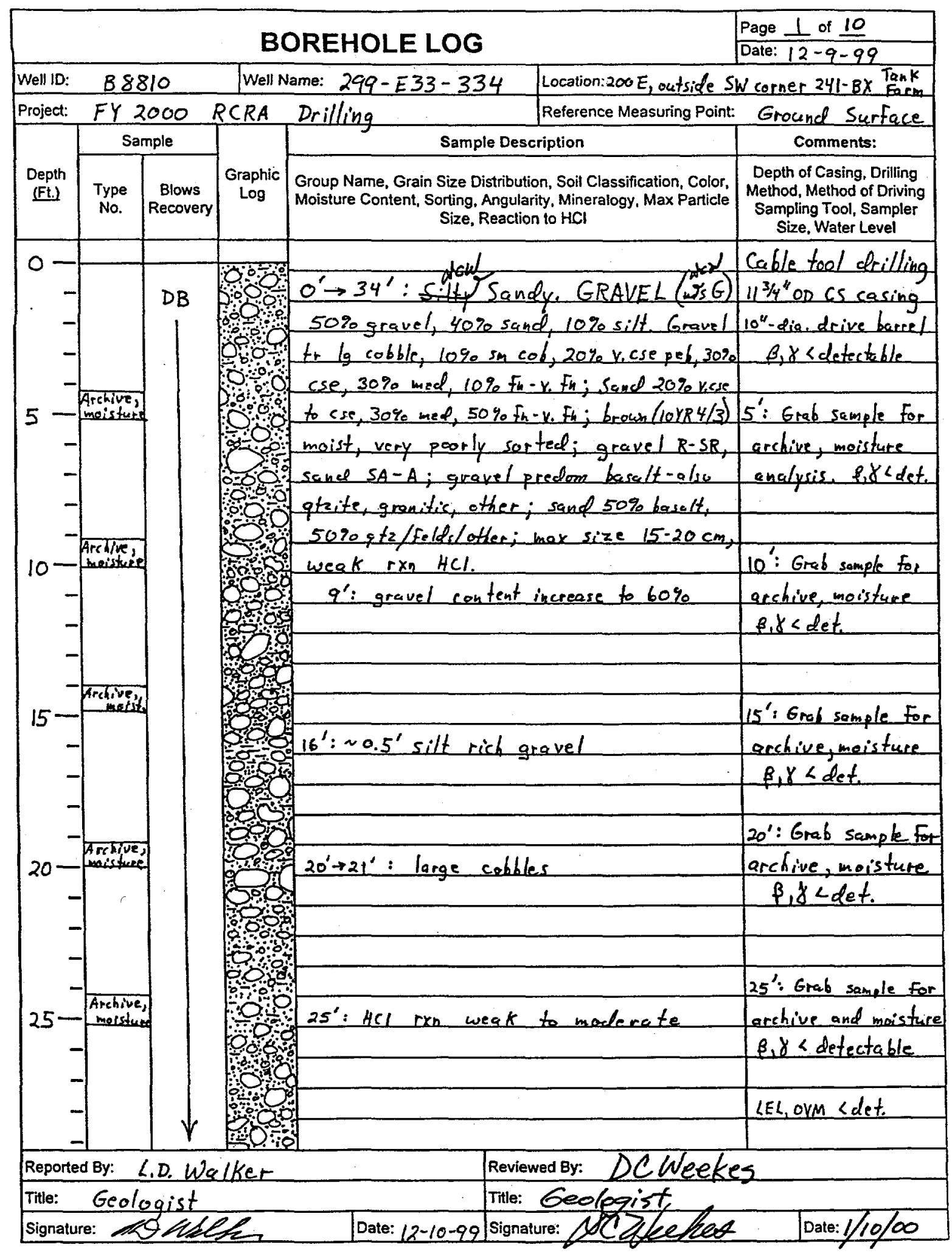




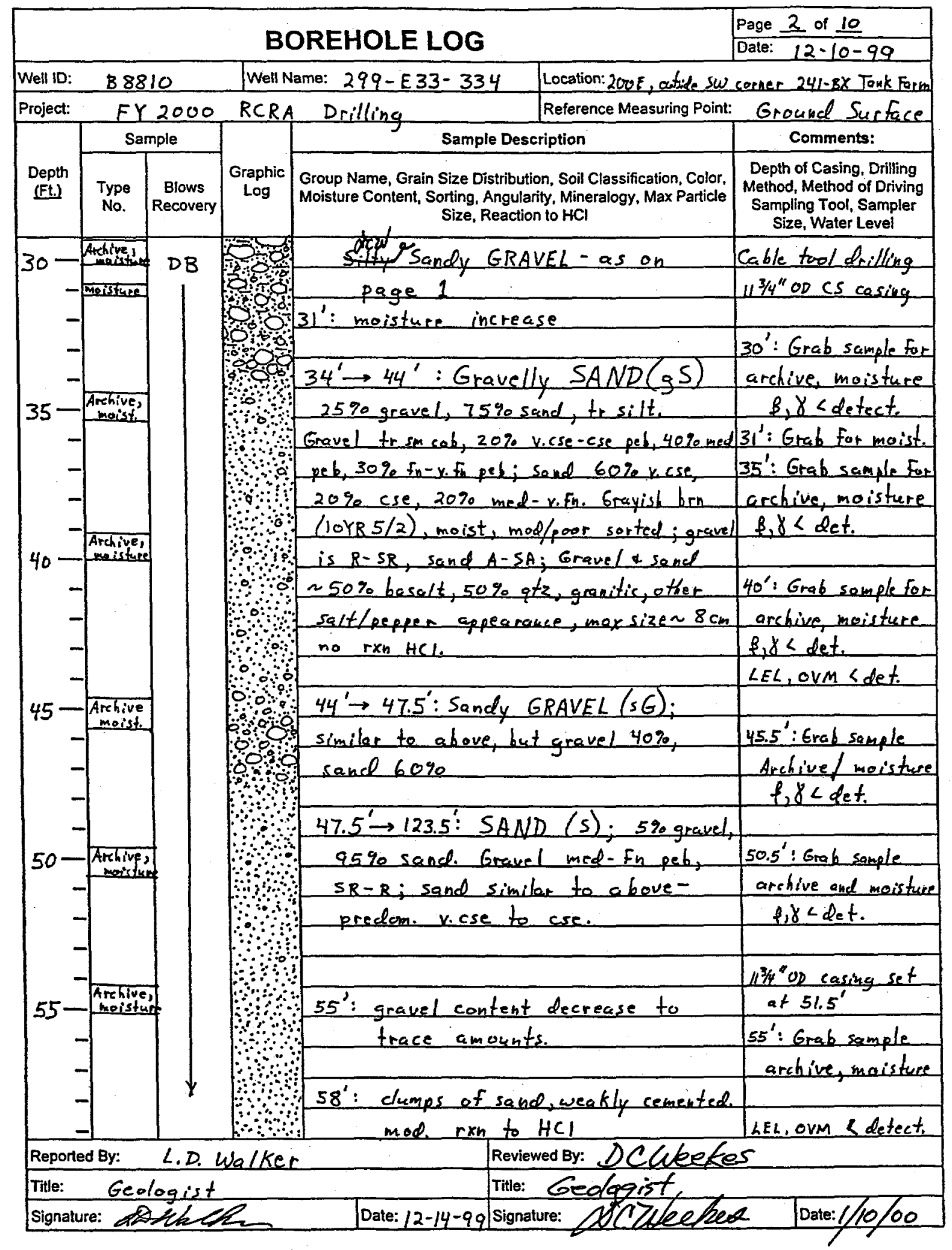




\begin{tabular}{|c|c|c|c|c|c|c|}
\hline \multirow{2}{*}{\multicolumn{6}{|c|}{ BOREHOLE LOG }} & Page 3 of 10 \\
\hline & & & & & & Date: $12-14-99$ \\
\hline Well ID: & \multicolumn{2}{|c|}{ B8810 } & \multicolumn{2}{|c|}{ Well Name: $299-E 33-334$} & \multicolumn{2}{|c|}{ Location: $200 E$, outside $S W$ corner $241-8 X \operatorname{Tan}_{B 2 n}$} \\
\hline Project: & \multicolumn{2}{|c|}{ FY 2000} & \multirow{2}{*}{\multicolumn{3}{|c|}{\begin{tabular}{l}
\multicolumn{2}{c|}{ Reference Measuring Point: } \\
Sample Description
\end{tabular}}} & Ground Surface \\
\hline \multirow[b]{2}{*}{$\begin{array}{l}\text { Depth } \\
\text { (Ft.) }\end{array}$} & \multicolumn{2}{|c|}{ Sample } & & & & Comments: \\
\hline & $\begin{array}{l}\text { Type } \\
\text { No. }\end{array}$ & $\begin{array}{c}\text { Blows } \\
\text { Recovery }\end{array}$ & $\begin{array}{c}\text { Graphic } \\
\text { Log }\end{array}$ & \multicolumn{2}{|c|}{$\begin{array}{c}\text { Group Name, Grain Size Distribution, Soil Classification, Color, } \\
\text { Moisture Content, Sorting, Angularity, Mineralogy, Max Particle } \\
\text { Size, Reaction to HCl }\end{array}$} & \begin{tabular}{|c|} 
Depth of Casing. Drilling \\
Method, Method of Driving \\
Sampling Tool, Sampler \\
Size, Water Level
\end{tabular} \\
\hline \multirow[t]{4}{*}{$60-\bar{T}$} & \multirow[t]{4}{*}{$\begin{array}{l}\text { Prechive } \\
\text { Sheisting }\end{array}$} & \multirow[t]{22}{*}{ DB } & \multirow{4}{*}{ (1) } & & & Coble tool drilling \\
\hline & & & & $\begin{array}{c}61.5^{\prime} \text { : Thin layet } \\
\text { then back }\end{array}$ & $\begin{array}{l}\left(\sim 0.2^{\prime}\right) \text { sandy silt, } \\
\text { to sand. }\end{array}$ & $85 \% " O D C S$ casing \\
\hline & & & & & & 60': Grab sample for \\
\hline & & & & & & archive and moisture \\
\hline \multirow[t]{4}{*}{$65-$} & \multirow[t]{4}{*}{\begin{tabular}{|l|l|} 
Archives \\
malist \\
\end{tabular}} & & \multirow{2}{*}{ 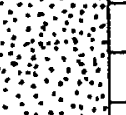 } & \multicolumn{2}{|c|}{$47.5^{\prime} \rightarrow 123.5^{\prime}:$ SAND $(S)$ tr gravel, } & \\
\hline & & & & \multicolumn{2}{|c|}{$100 \%$ sand tr silt. Sand is $10 \%$} & 65: Grab sample for \\
\hline & & & 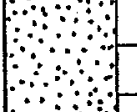 & \multicolumn{2}{|c|}{ Gray brom $(10 \mathrm{rR} 5 / 2)$, s/moist to dry, } & archive, moisture \\
\hline & & & $\begin{array}{lll}\because \\
\because \\
\because\end{array}$ & \multicolumn{2}{|c|}{ mod sorted, $S_{A}-A ; 30-4090$ besalt, } & \\
\hline \multirow{3}{*}{$\begin{array}{r}70- \\
- \\
-\end{array}$} & Arehives & & 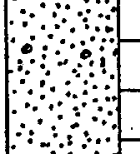 & \multicolumn{2}{|c|}{$\begin{array}{l}\text { at } 70 \text { feet Max size } \simeq 8 \text { mm; weak } \\
\text { HCl rxh. Tr fn-r. fn peb. at } 70^{\prime}-7 c^{\prime}\end{array}$} & $\begin{array}{l}70^{\prime}: \text { Grab sample- } \\
\text { arclive, moisture. }\end{array}$ \\
\hline & & & $\because$ & \multicolumn{2}{|c|}{-2} & \\
\hline & arentive & & 4 & \multicolumn{2}{|c|}{$75^{\prime}$ : moisture increase - dry to moist. } & $75^{\prime}$ : Grab sample - \\
\hline \multirow{3}{*}{$\begin{array}{rr}75 & - \\
& - \\
& - \\
& -\end{array}$} & & & 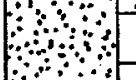 & & & archive, moisture. \\
\hline & & & $\because$ & & & \\
\hline & & & $\because 0^{\prime}$ & & & \\
\hline $80-$ & \begin{tabular}{|l|} 
Anchive, \\
moisture
\end{tabular} & & $\because$ & & & 80: Grat sample- \\
\hline & & & 6 & $80^{\prime}:$ & & archire, moisture \\
\hline & & & at & sand predom. $c$ & ese; otherwise as aboue. & $\beta, \gamma<$ detect. \\
\hline & & & $\because$ & & & \\
\hline $85-$ & $\begin{array}{l}\text { Archive, } \\
\text { moisture }\end{array}$ & & Ot & & & 85': Grab sample- \\
\hline & & & 6 & & & archive, moisture \\
\hline & & & ot & & & \\
\hline & & & 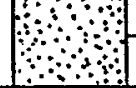 & & & \\
\hline Reported & d By: & L.D. $\mathrm{n}$ & jalker & & DCuket & 6 \\
\hline Title: & Geal & logist & & & Title: Gedogist & \\
\hline Signature & e: $\geq$ & Q Whele & & Date: $12 / 14 / 99$ & signature: $x$ Dee hes & Date: $1 / 10 / 00$ \\
\hline
\end{tabular}

BHI-EE-183 (12/9n 


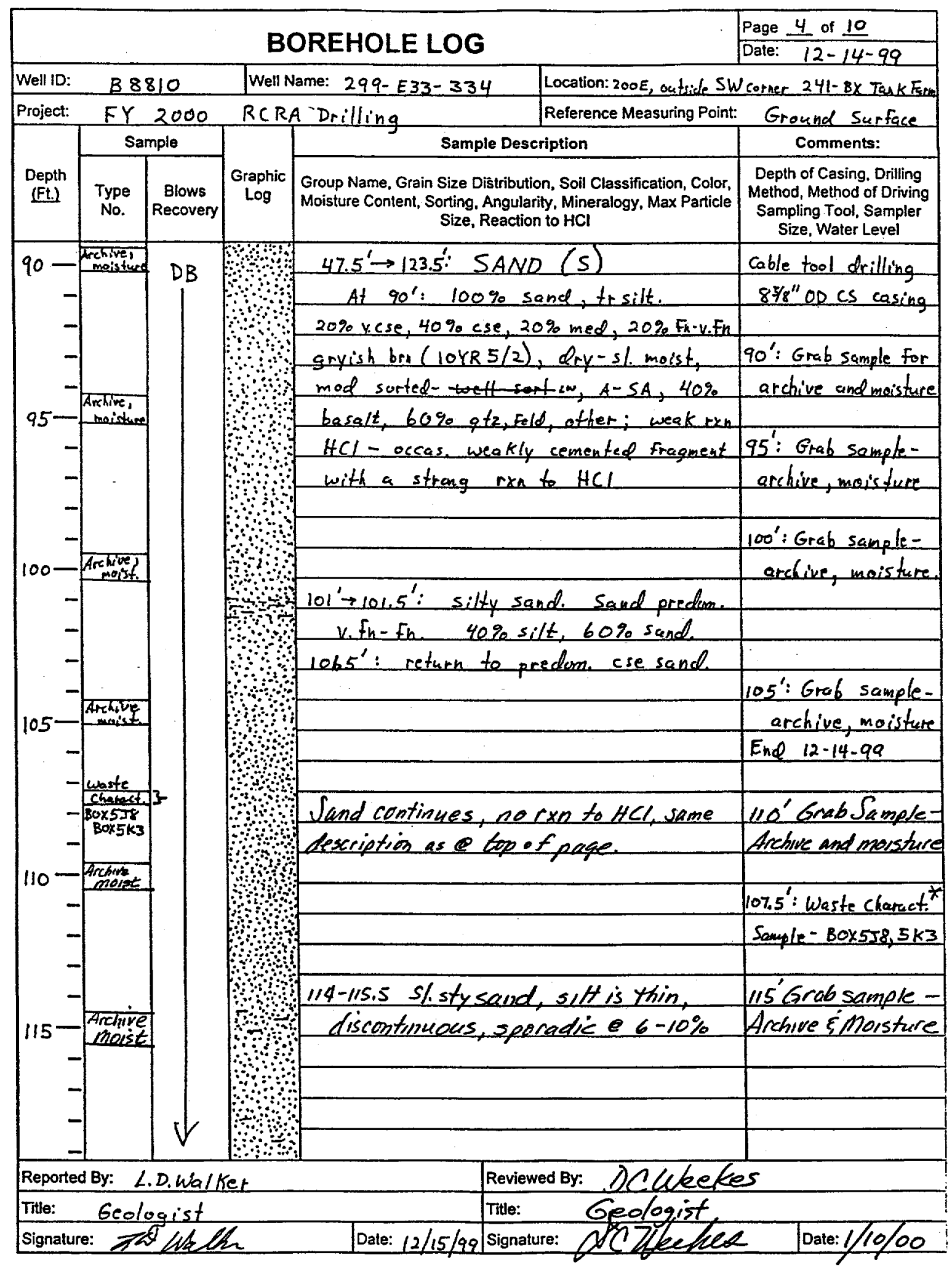




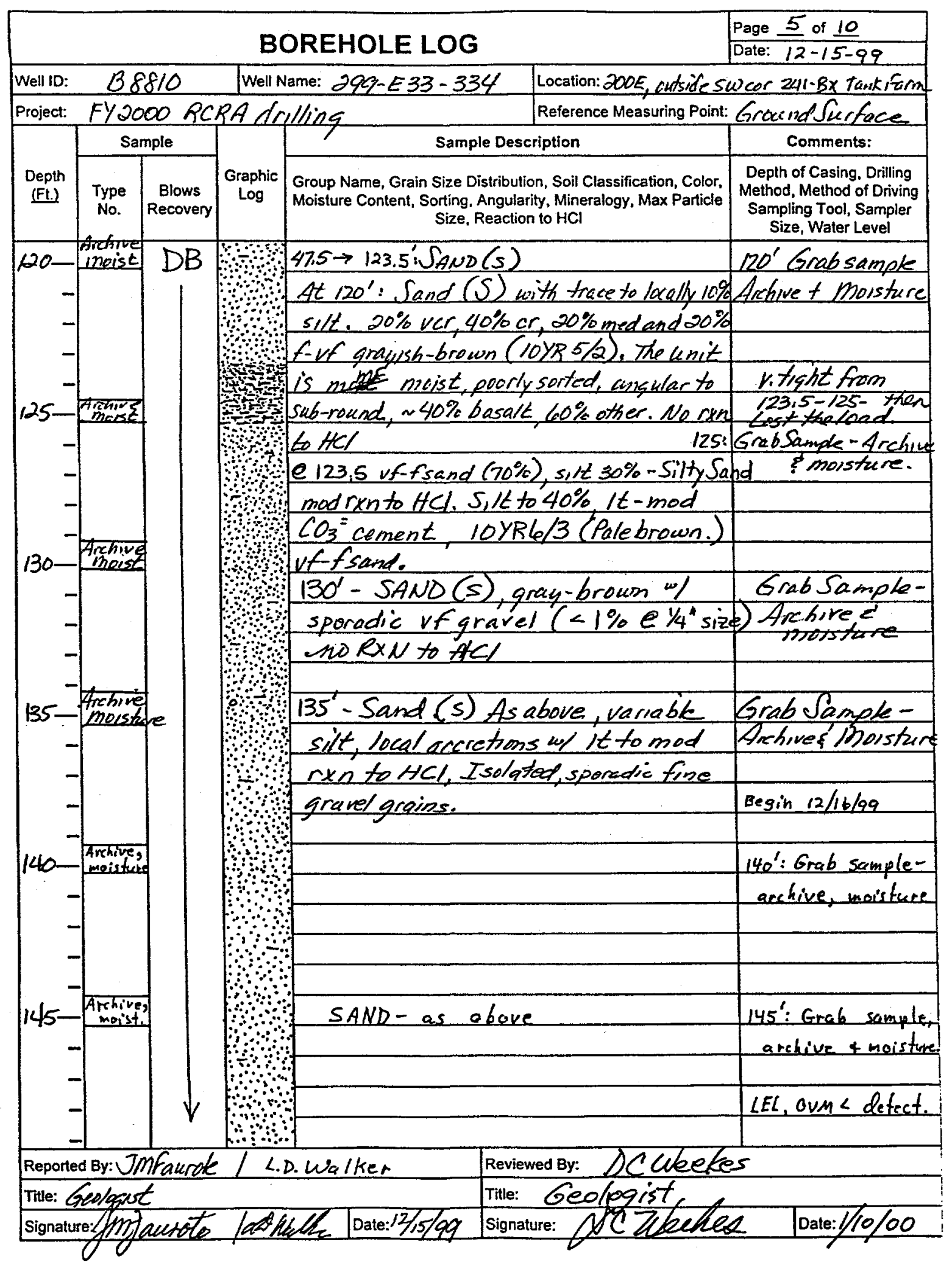




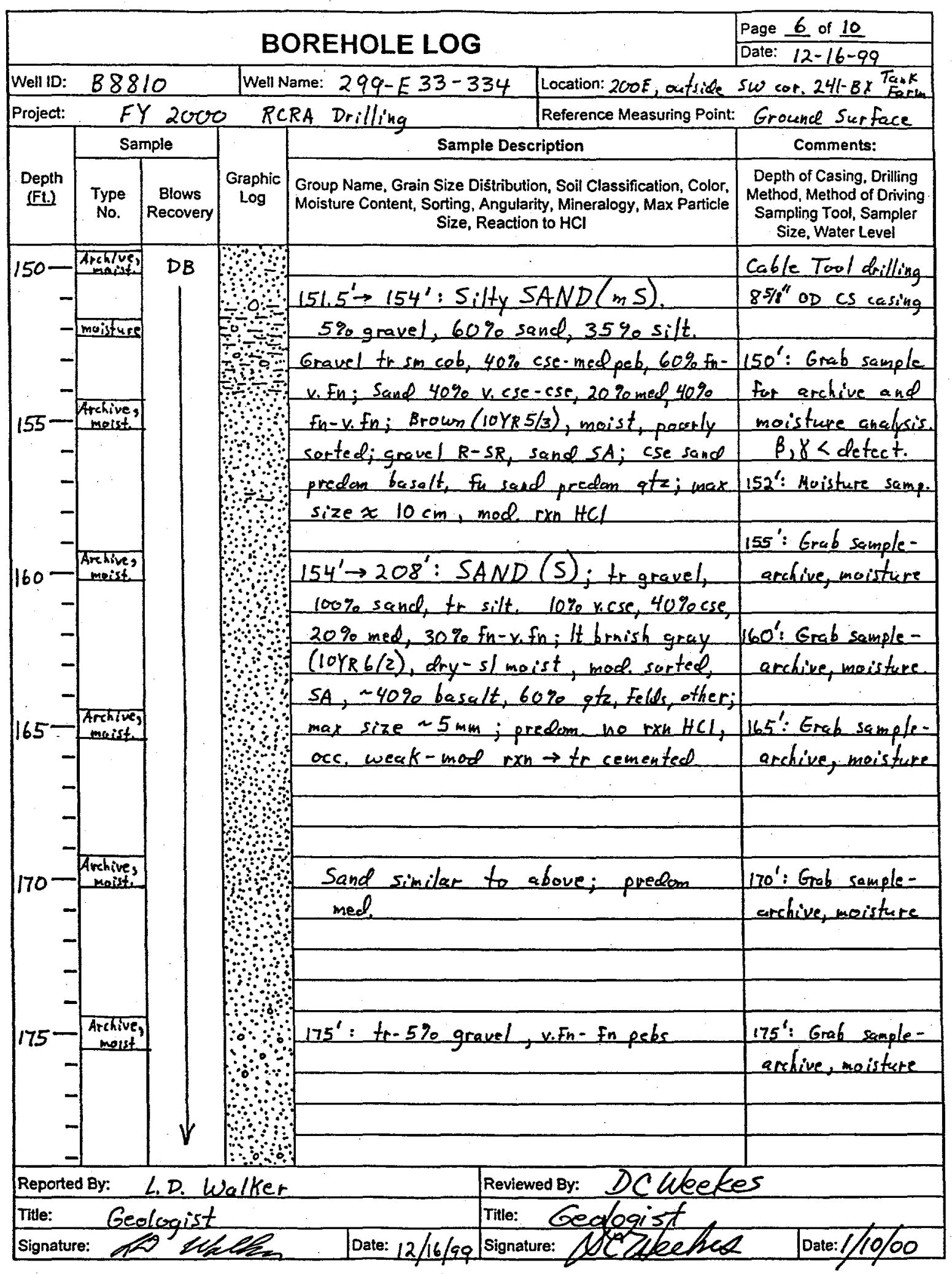




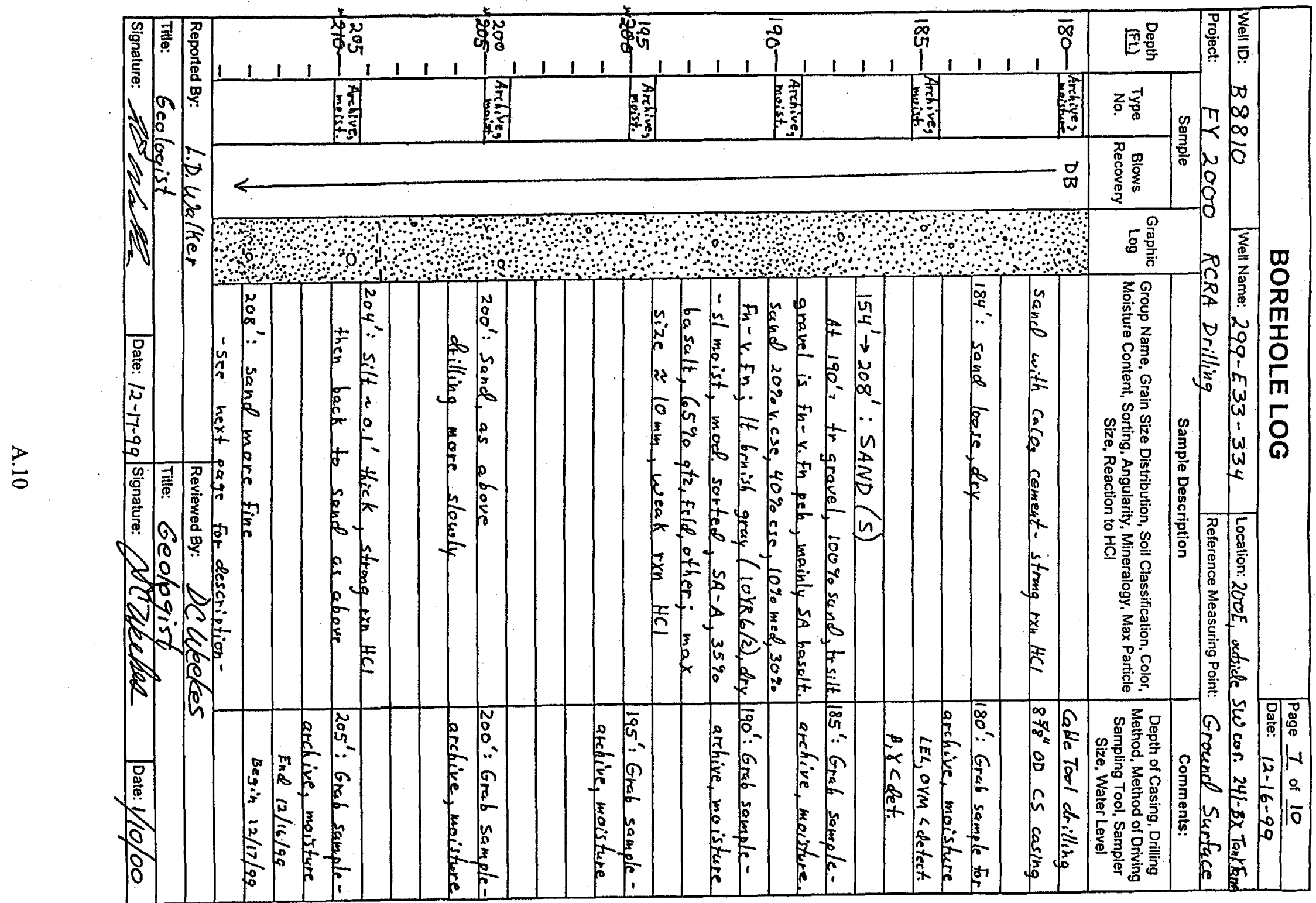




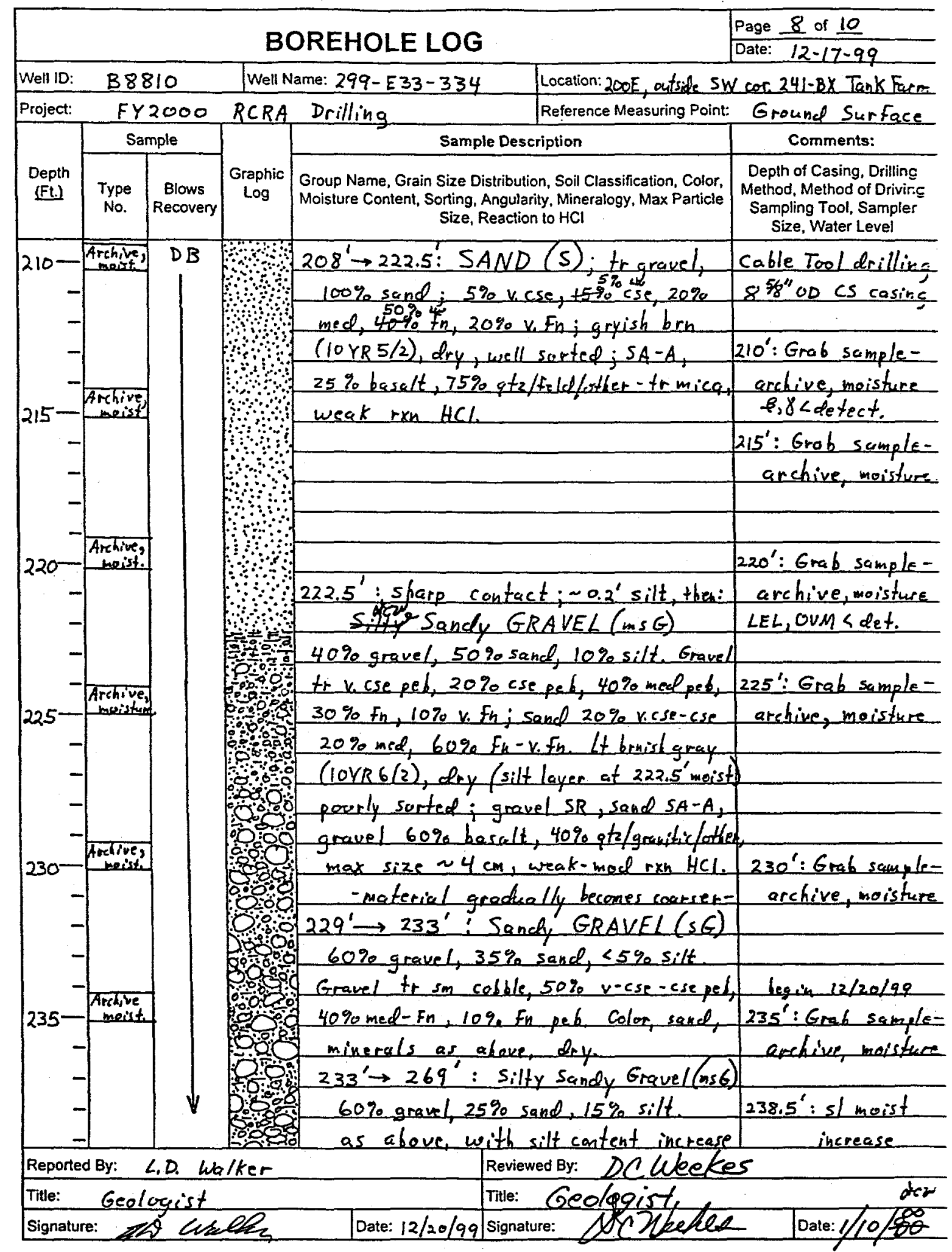

BHI-EE-183 (12/97) 


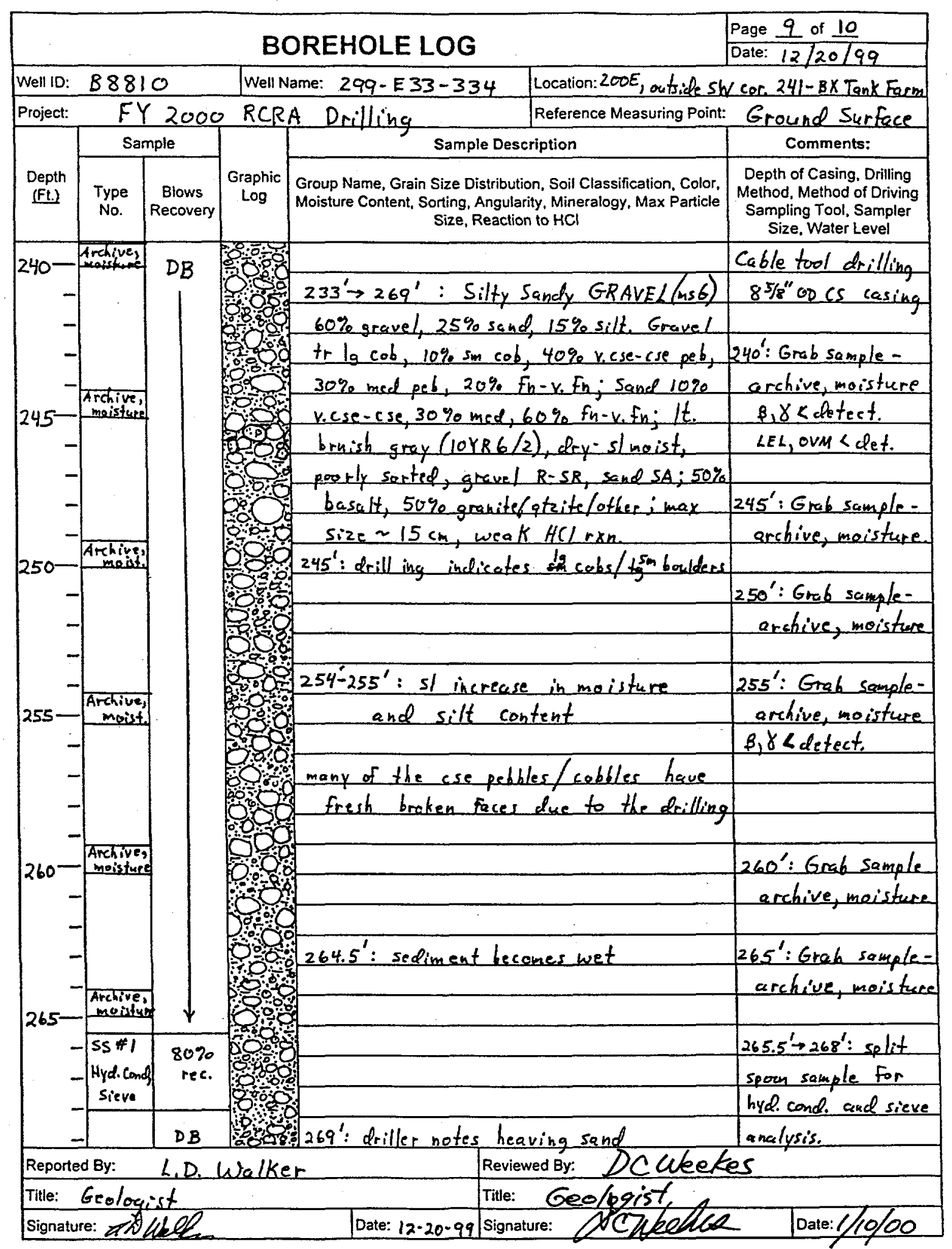

BHI-EE-183 (12/97) 


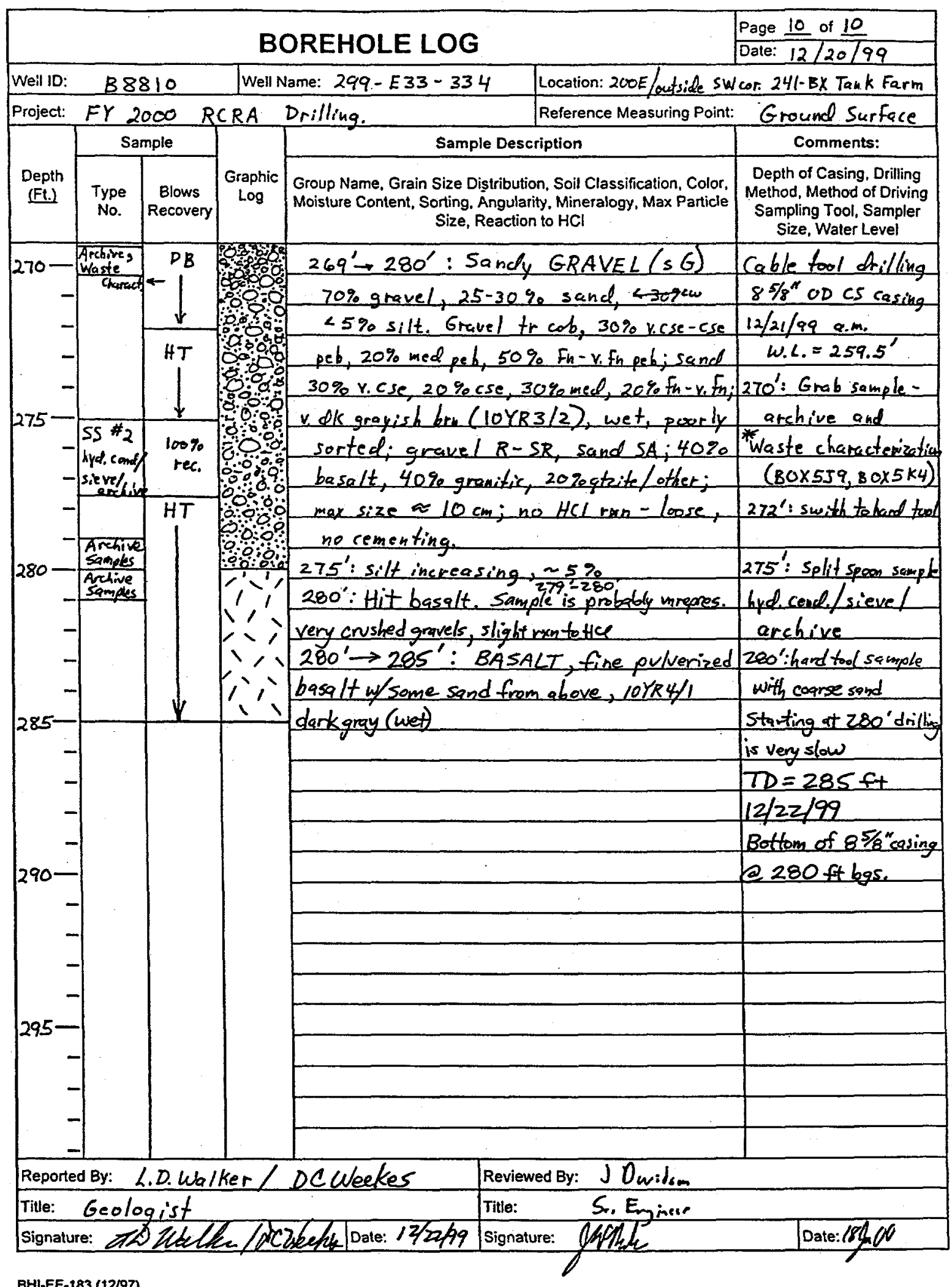




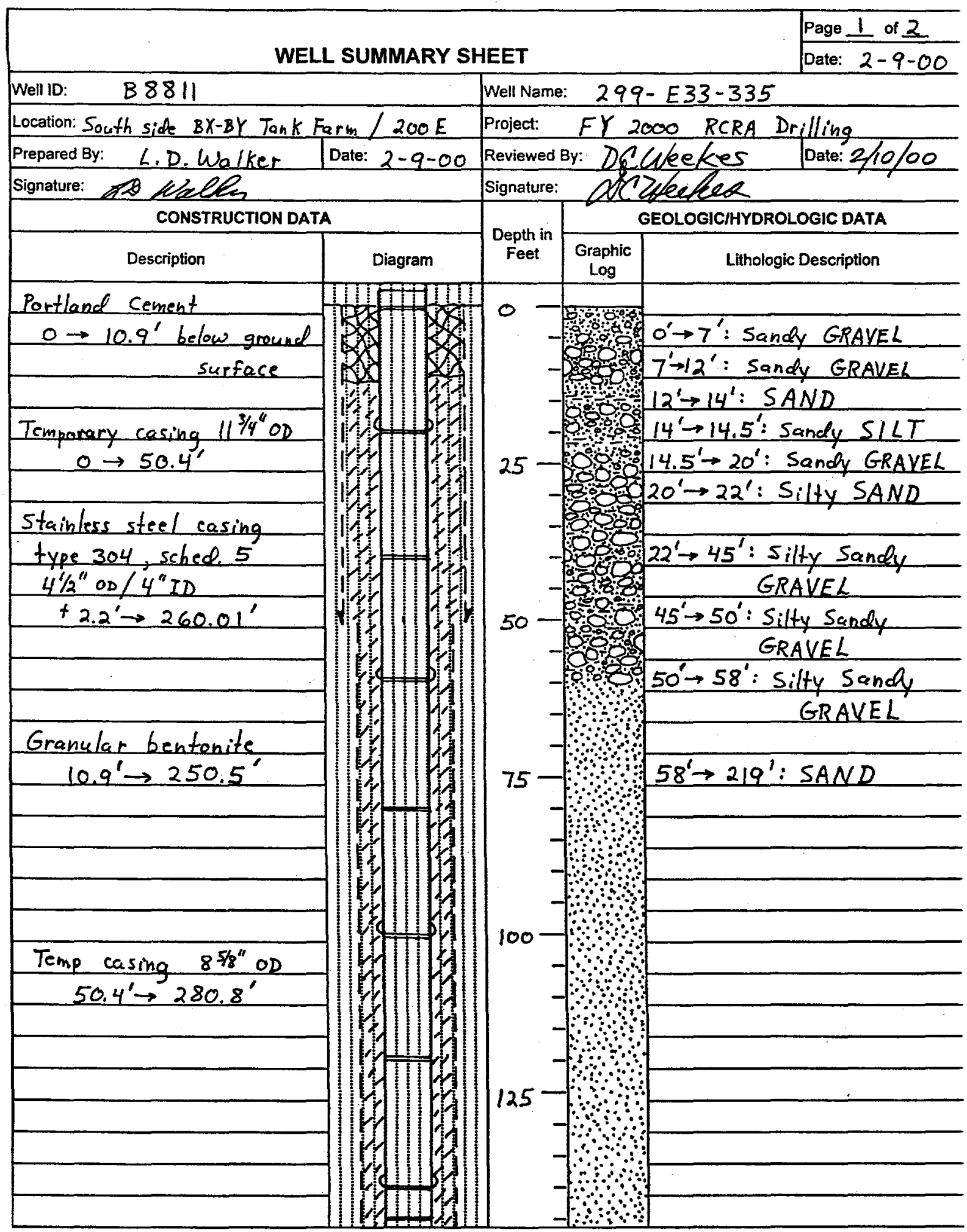

BHI-EE-189 (12/97) 


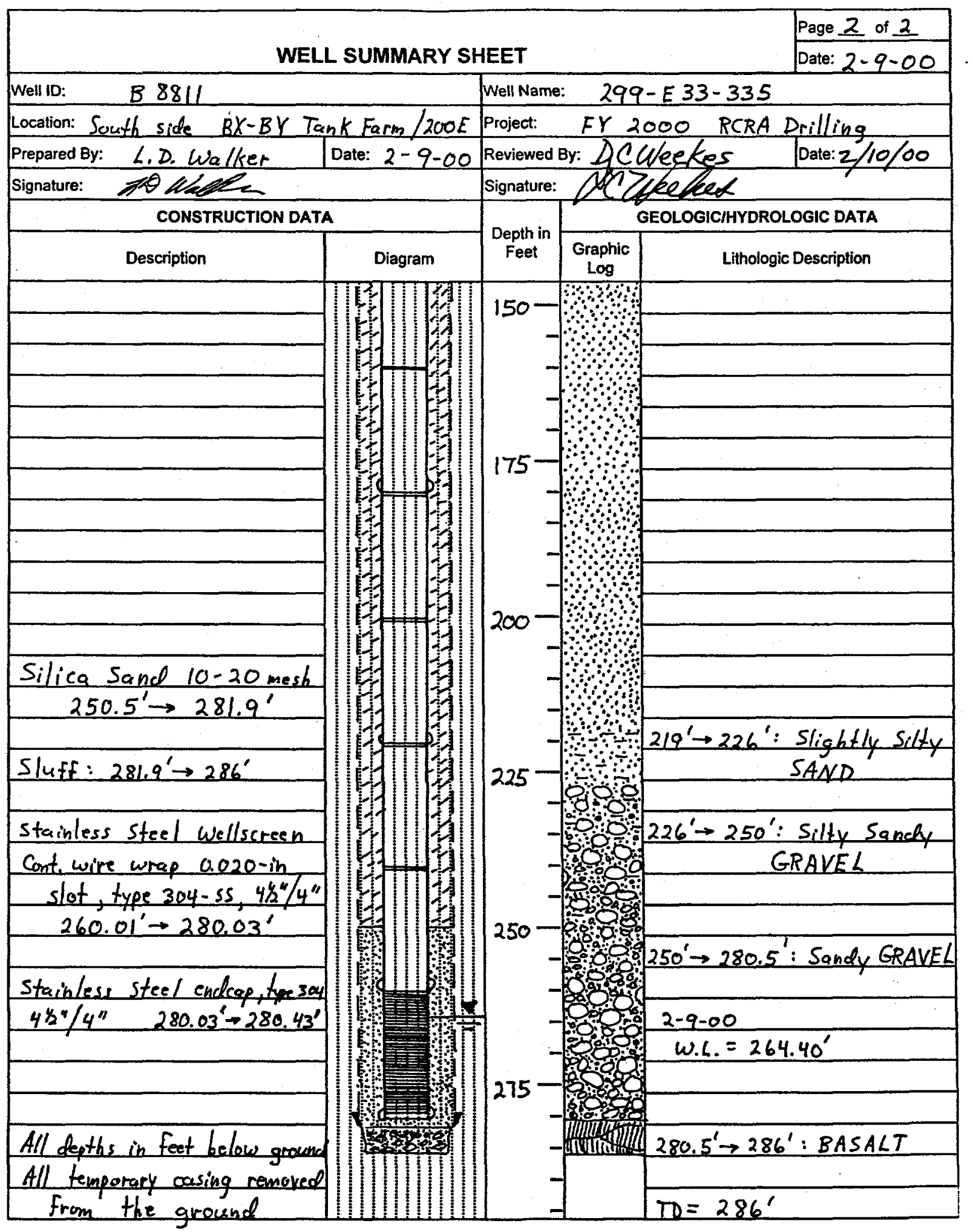

BHLEE-189 (1297) 


\title{
WELL CONSTRUCTION SUMMARY REPORT
}

\author{
Start Date: $\quad 12-2-99$ \\ Finish Date: $2-10-00$ \\ Page $\perp$ of 1
}

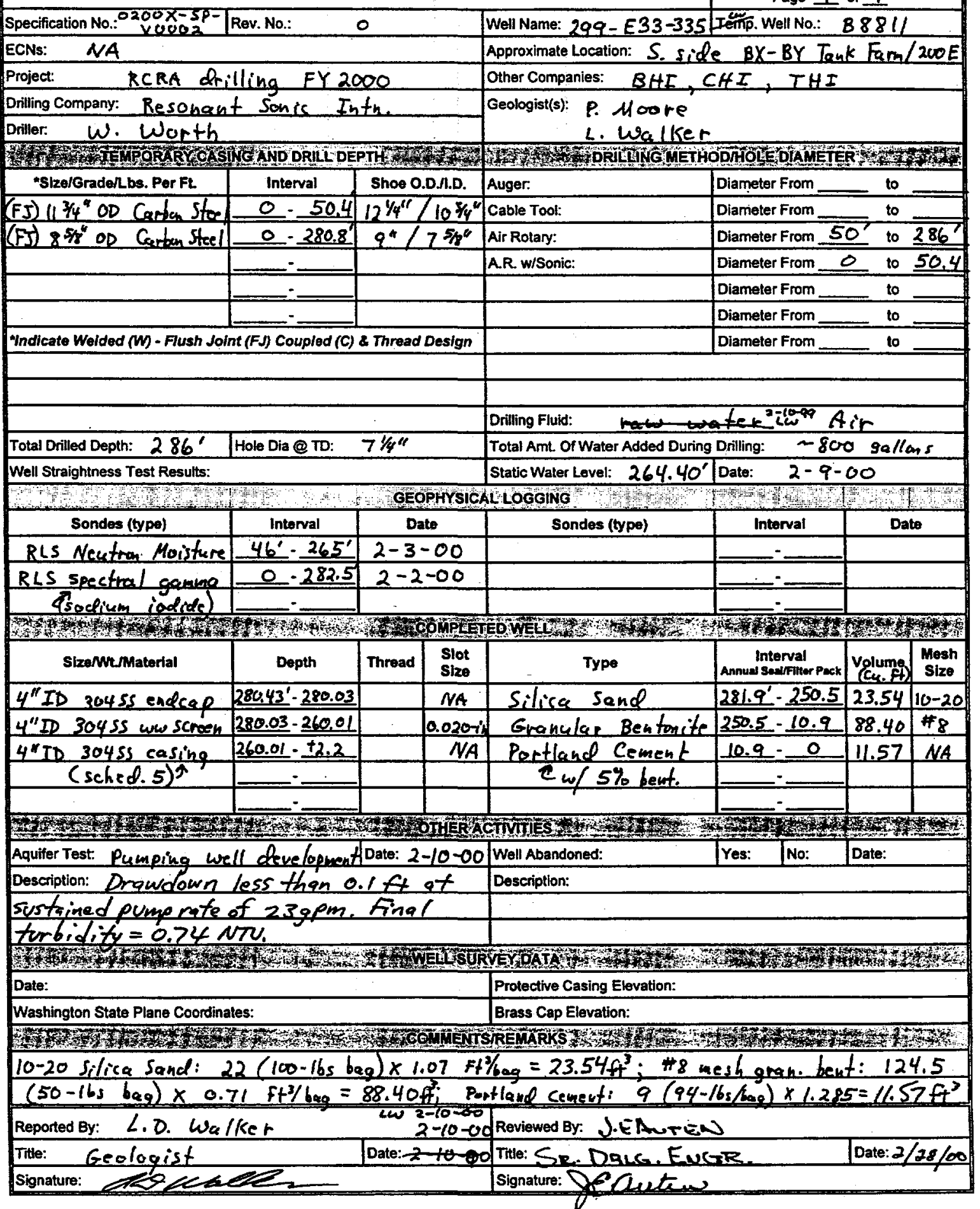

BHI-EE-181 (12/97) 


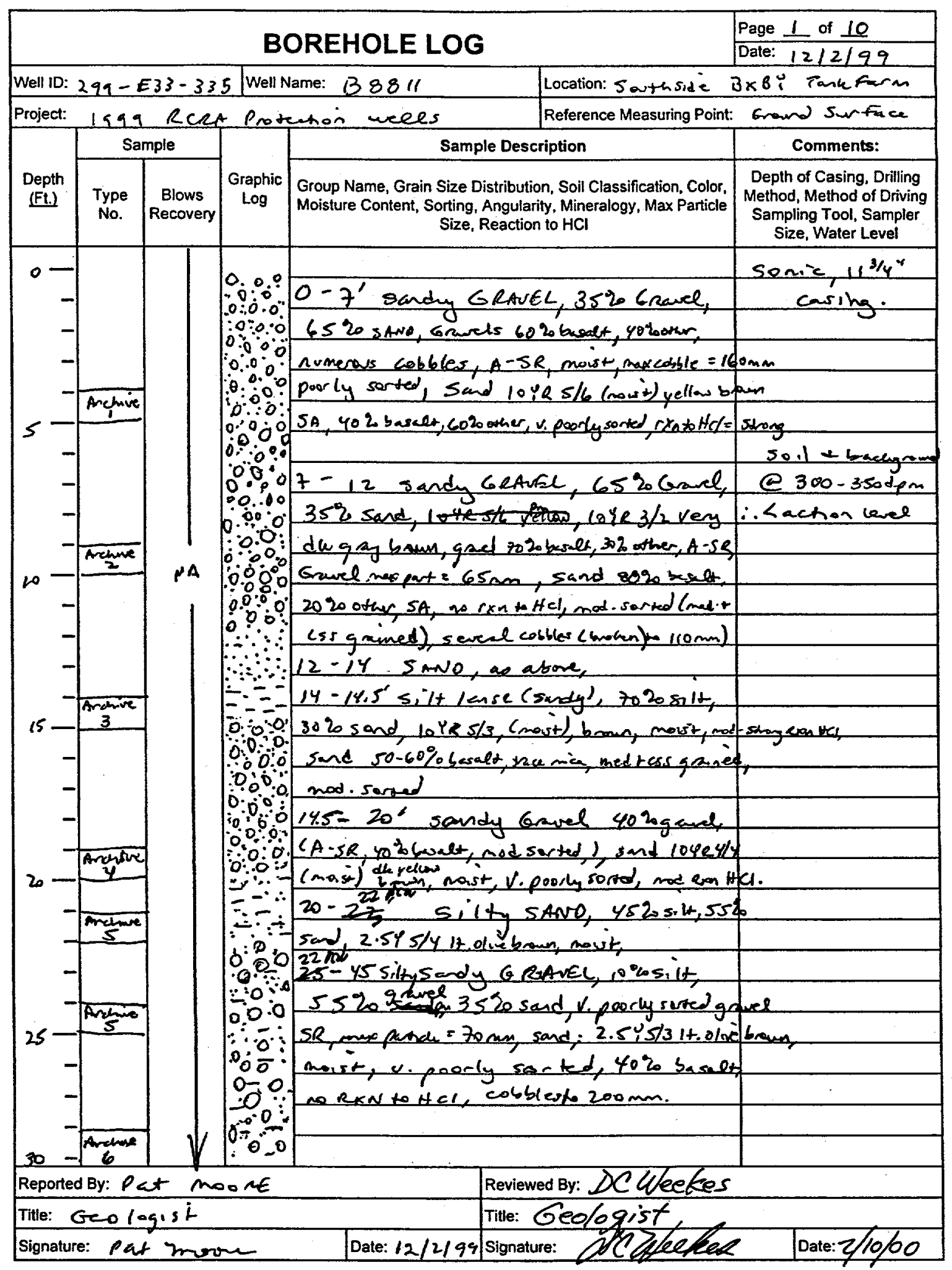

BHI-EE-183 (12/97) 


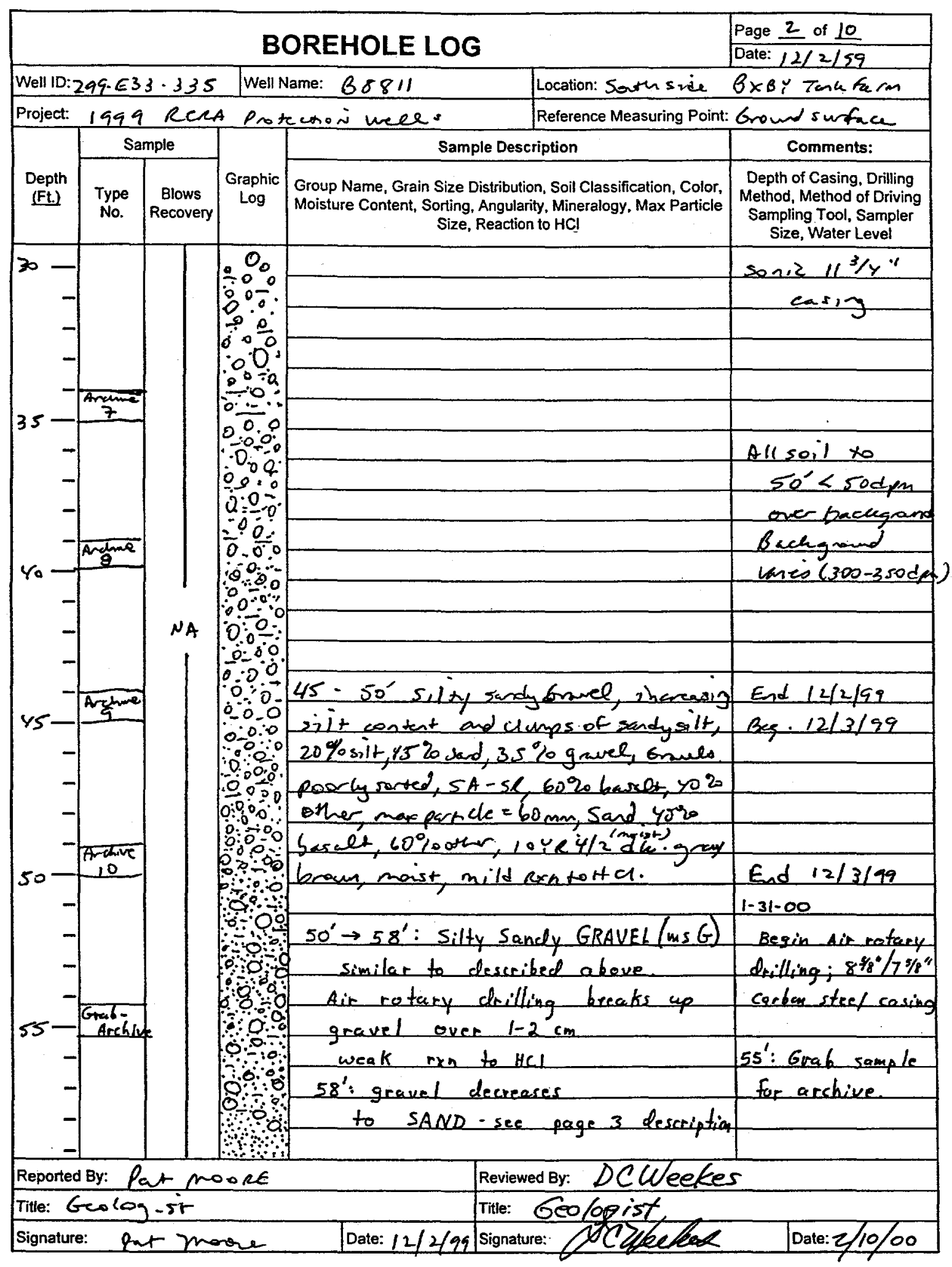

BHI-EE-183 (12/97) 


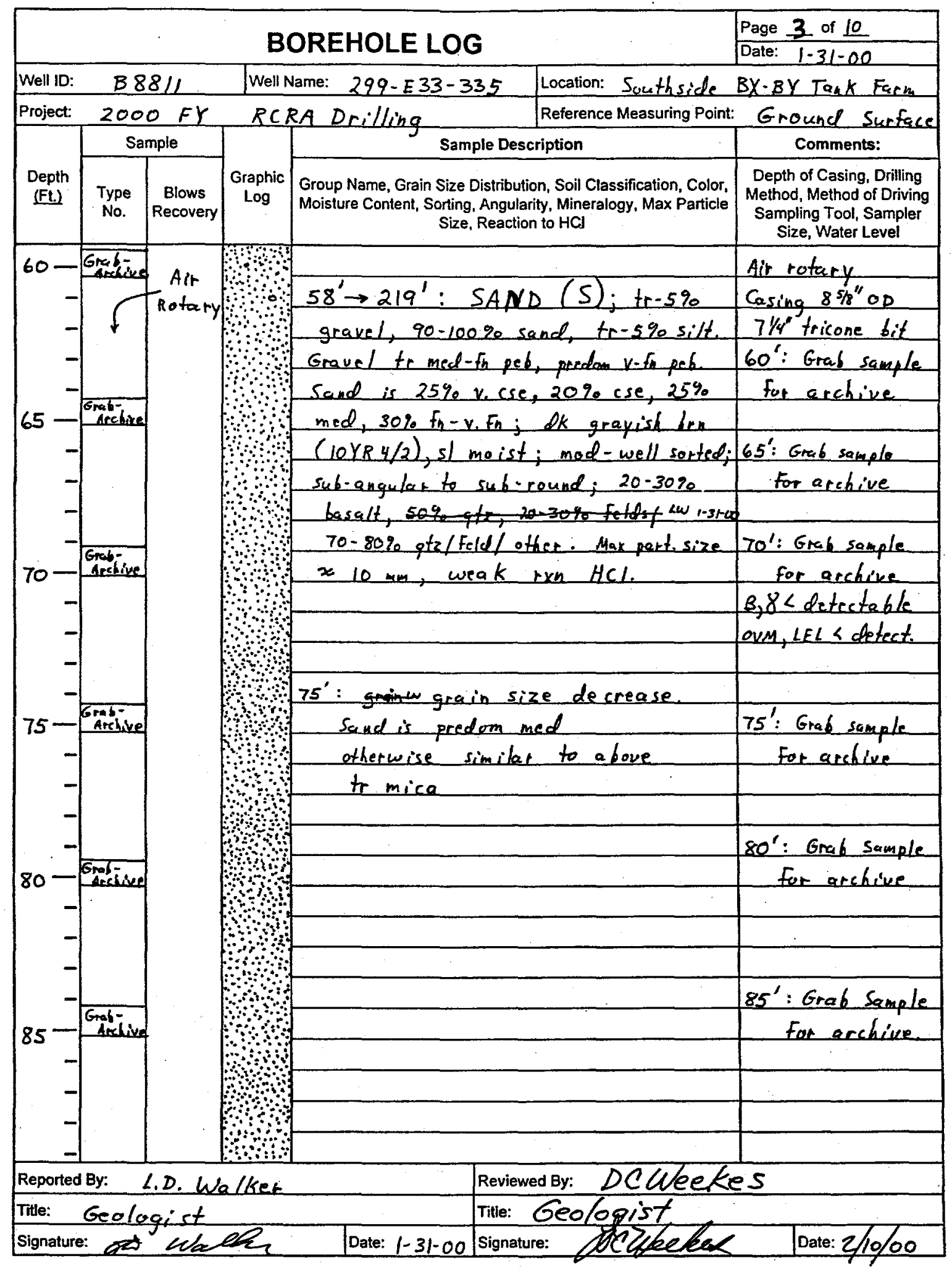

BHI-EE-183 (12/97) 


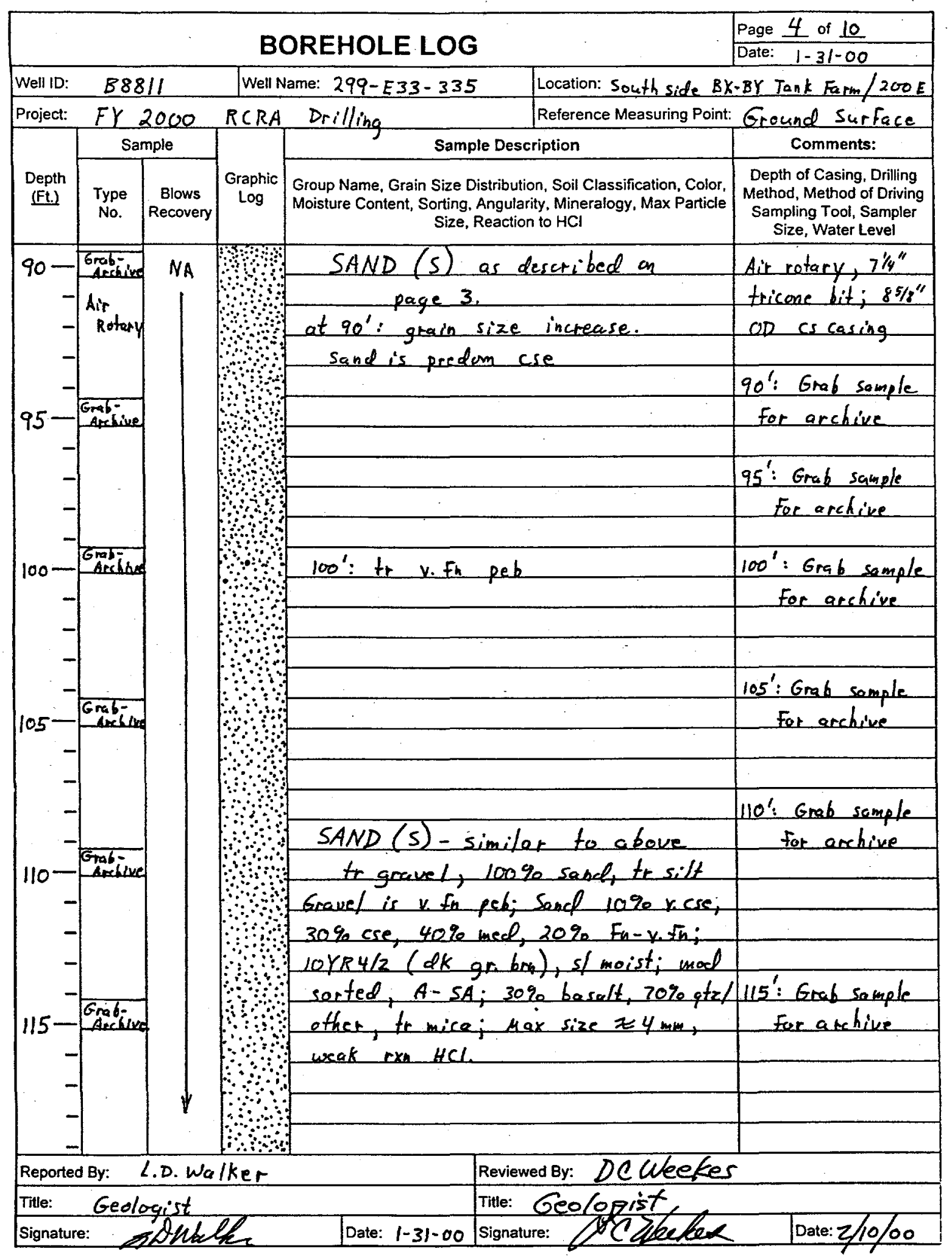

BHI-EE-183 (12/97) 


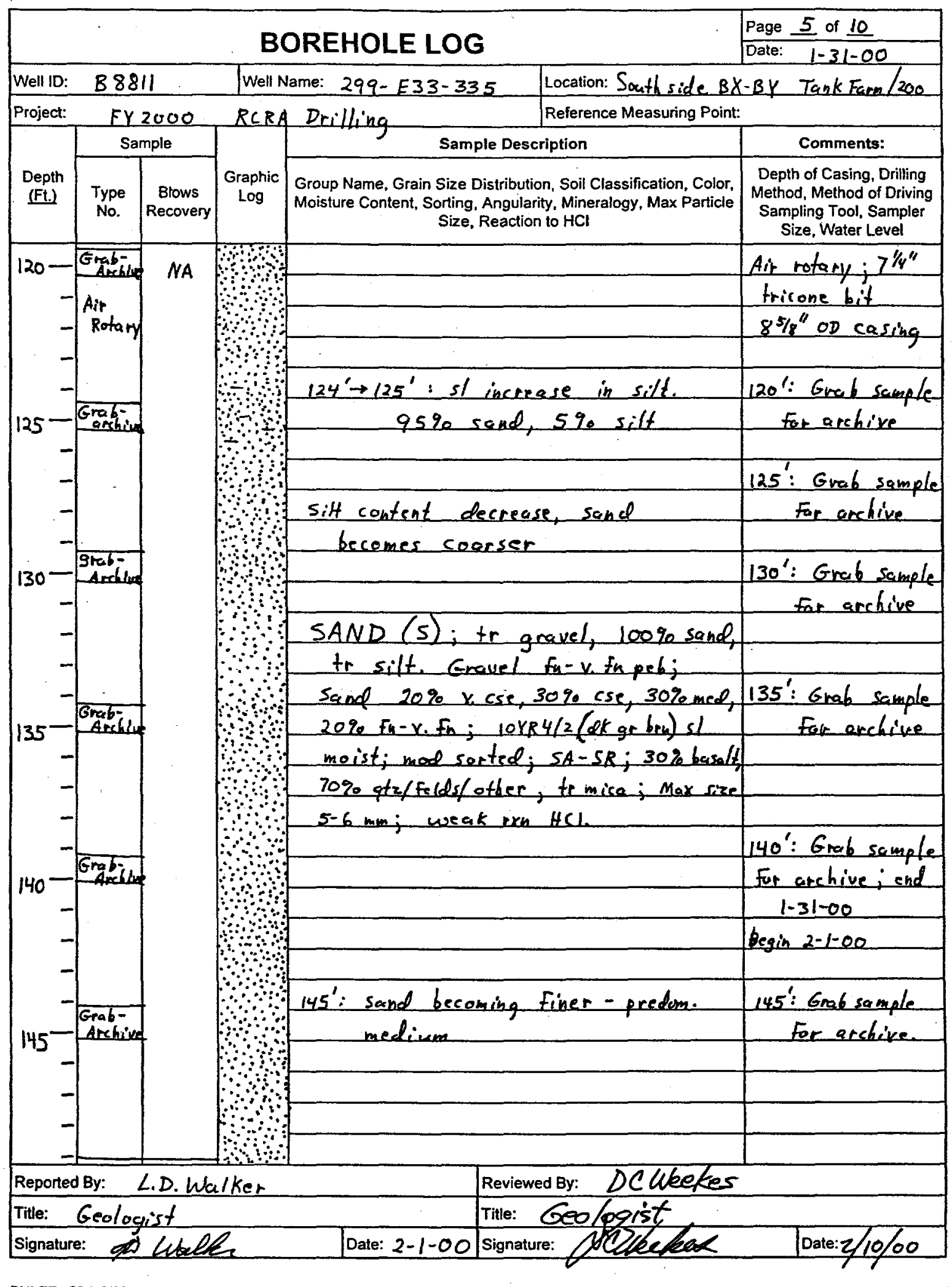

BHH-EE-183 (12197) 


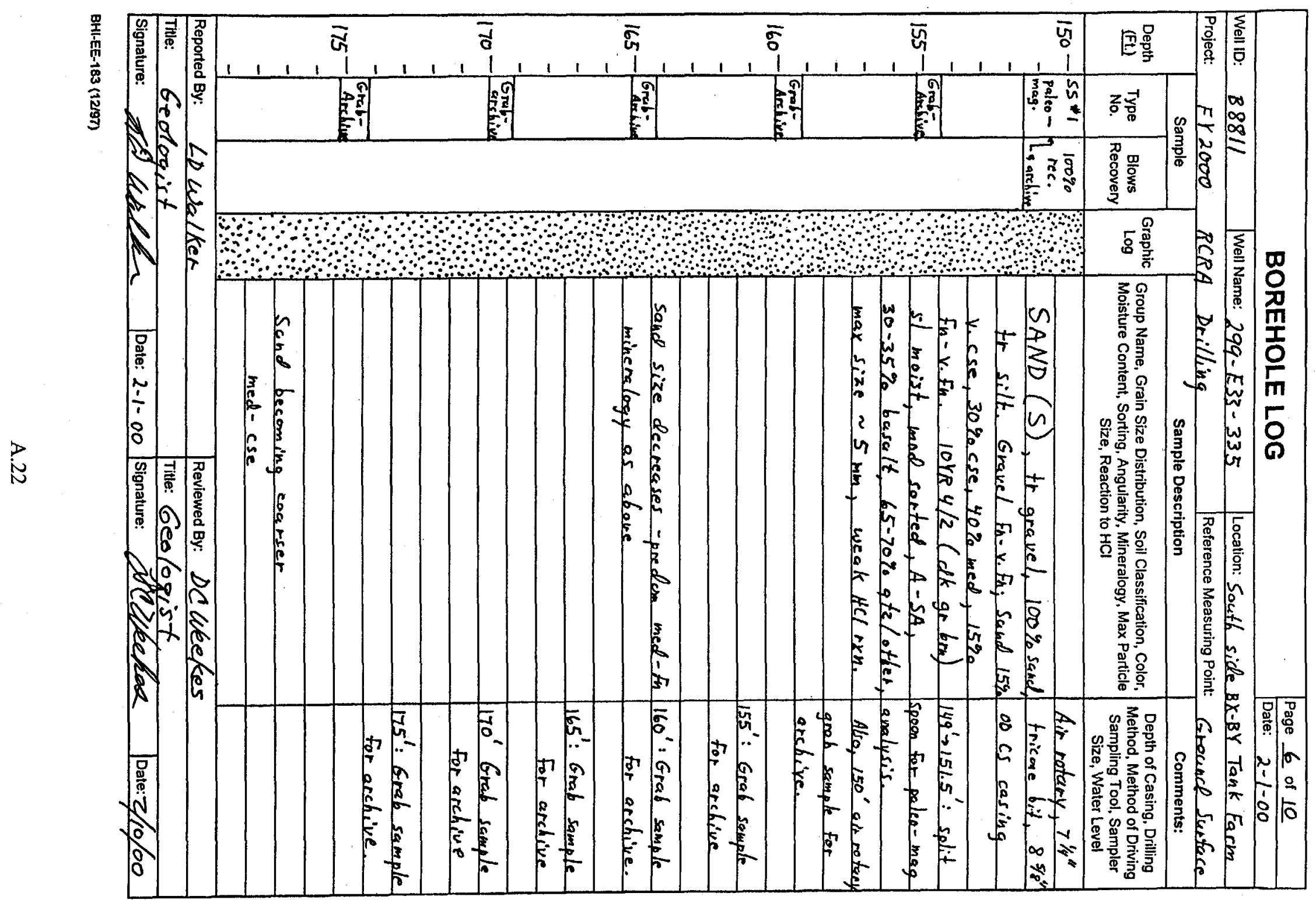




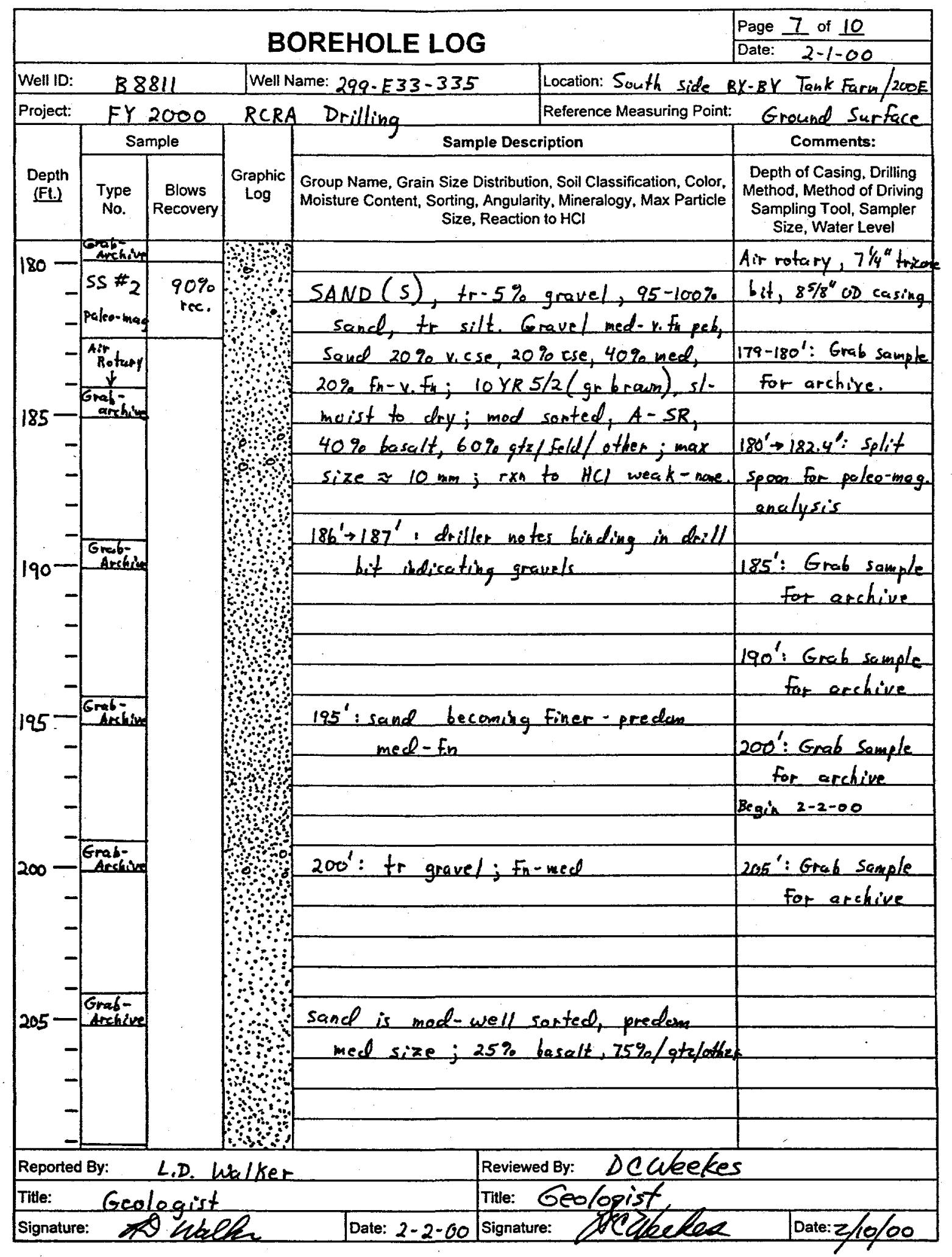

BH-EE-183 (1297) 


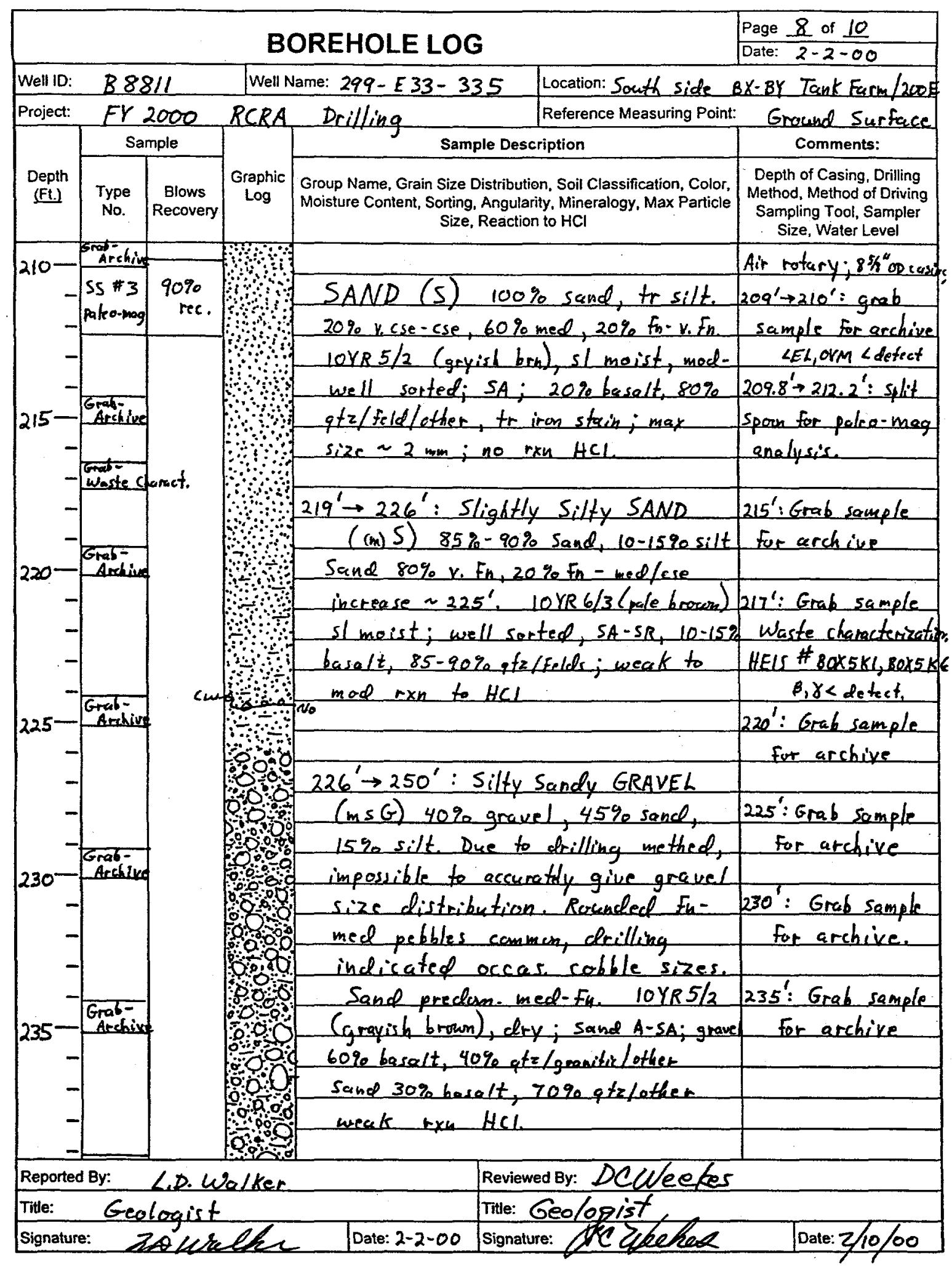

BH-EE-183 (12/97) 


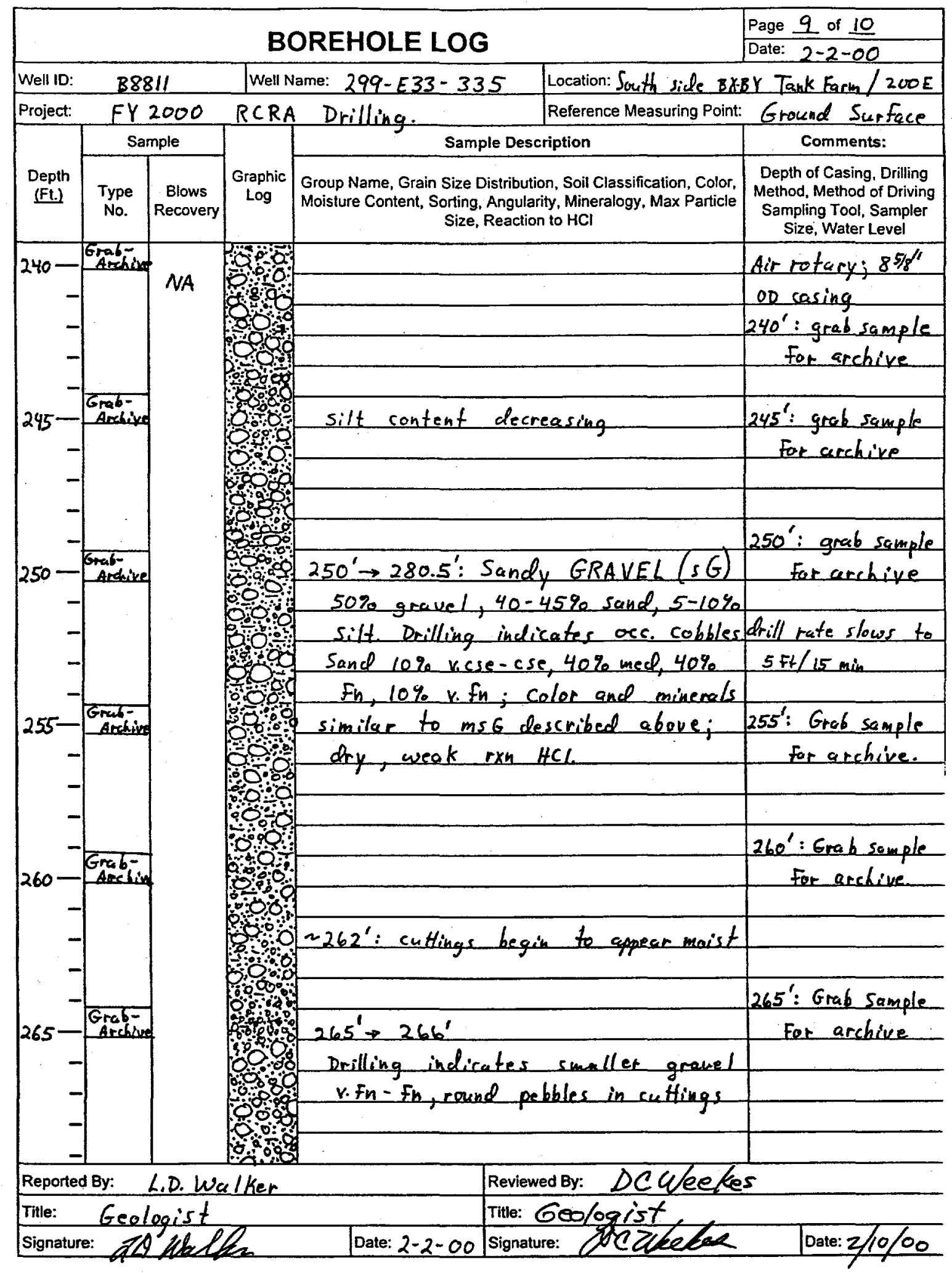

BHI-EE-183 (12/97) 


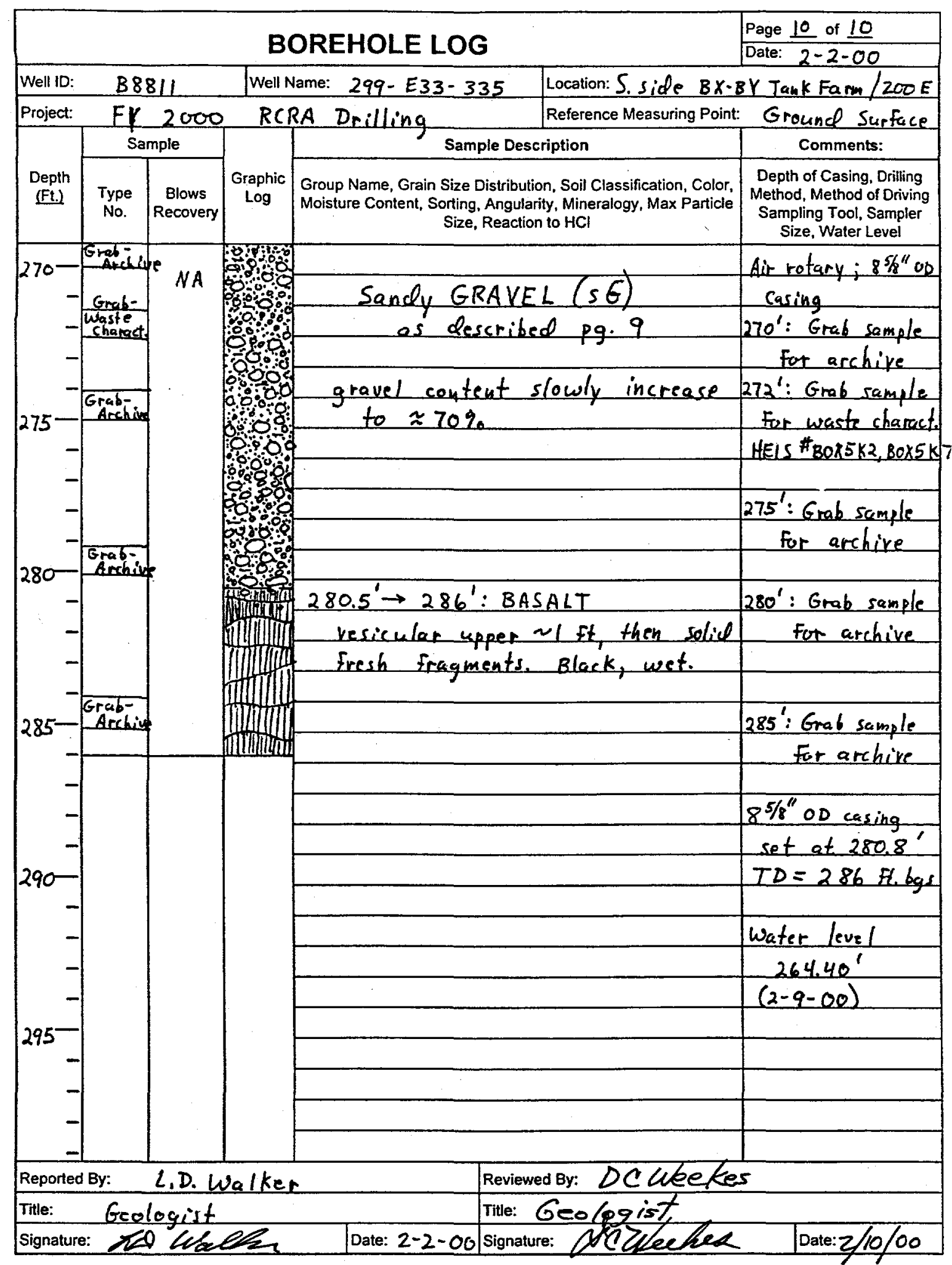

BHI-EE-183 (12/97) 
Appendix B

Moisture Content for Samples from Well 299-E33-334 


\section{Appendix B}

\section{Moisture Content for Samples from Well 299-E33-334}

This appendix includes the results of laboratory testing for moisture content of samples collected from well 299-E33-334. Moisture content was measured as weight loss after drying an aliquot of the bulk sample at $105^{\circ} \mathrm{C}$ for 24 hours or until weight was constant for two consecutive measurements. The measurements were done in the Applied Geology and Geochemistry Group laboratory under the supervision of Mr. Jeff Serne.

Table B.1. Moisture Content of Sediment Samples from Borehole 299-E33-334

\begin{tabular}{||c|c||c|c||c|c||}
\hline \multicolumn{7}{|c|}{ 299-E33-334 } \\
\hline $\begin{array}{c}\text { Depth } \\
\text { (ft bgs) }\end{array}$ & $\begin{array}{c}\text { Moisture } \\
\text { Content } \\
\text { (wt \%) }\end{array}$ & $\begin{array}{c}\text { Depth } \\
\text { (ft bgs) }\end{array}$ & $\begin{array}{c}\text { Moisture } \\
\text { Content } \\
\text { (wt \%) }\end{array}$ & $\begin{array}{c}\text { Depth } \\
\text { (ft bgs) }\end{array}$ & $\begin{array}{c}\text { Moisture } \\
\text { Content } \\
\text { (wt \%) }\end{array}$ \\
\hline \hline 5 & 4.14 & 95 & 3.12 & 185 & 2.59 \\
\hline 10 & 4.24 & 100 & 3.03 & 190 & 2.79 \\
\hline 15 & 3.91 & 105 & 3.25 & 195 & 3.23 \\
\hline 20 & 5.86 & 110 & 3.74 & 200 & 3.15 \\
\hline 25 & 6.90 & 115 & 3.43 & 205 & 2.92 \\
\hline 30 & 6.14 & 120 & 3.44 & 210 & 3.63 \\
\hline 31 & 11.19 & 125 & 6.69 & 215 & 3.48 \\
\hline 35 & 3.38 & 130 & 2.16 & 220 & 3.21 \\
\hline 40 & 3.58 & 135 & 2.25 & 225 & 2.79 \\
\hline 45.5 & 3.11 & 140 & 2.39 & 230 & 2.63 \\
\hline 50.5 & 2.17 & 145 & 2.40 & 235 & 2.65 \\
\hline 55 & 2.24 & 150 & 2.42 & 240 & 2.99 \\
\hline 60 & 3.06 & 152 & 8.00 & 245 & 2.93 \\
\hline 65 & 2.05 & 155 & 2.68 & 250 & 3.16 \\
\hline 70 & 2.19 & 160 & 2.74 & 255 & 3.66 \\
\hline 75 & 2.39 & 165 & 2.68 & 260 & 2.88 \\
\hline 80 & 2.45 & 170 & 2.84 & 265 & 5.24 \\
\hline 85 & 2.99 & 175 & 2.63 & & \\
\hline 90 & 2.71 & 180 & 2.70 & & \\
\hline & & & & & \\
\hline
\end{tabular}

B.1 
Appendix C

Borehole Geophysical Logs 


\section{Appendix C}

\section{Borehole Geophysical Logs}

This appendix contains the NaI spectral gamma-ray and neutron-neutron moisture logs for wells 299-E33-334 and 299-E33-335. The logs were run and analyzed by Waste Management Federal Services Northwest, Inc. Log Header sheets and Log Analysis Summary Reports are included with the logs. 


\section{RLS Scintillator Gamma Ray Borehole Survey \\ Waste Management Technical Services}

\section{Log Header}

Project: PNNL RCRA

Well: 299-E33-334

\section{Log Type: $\quad$ Nal Spectral Gamma Ray}

Borehole Information

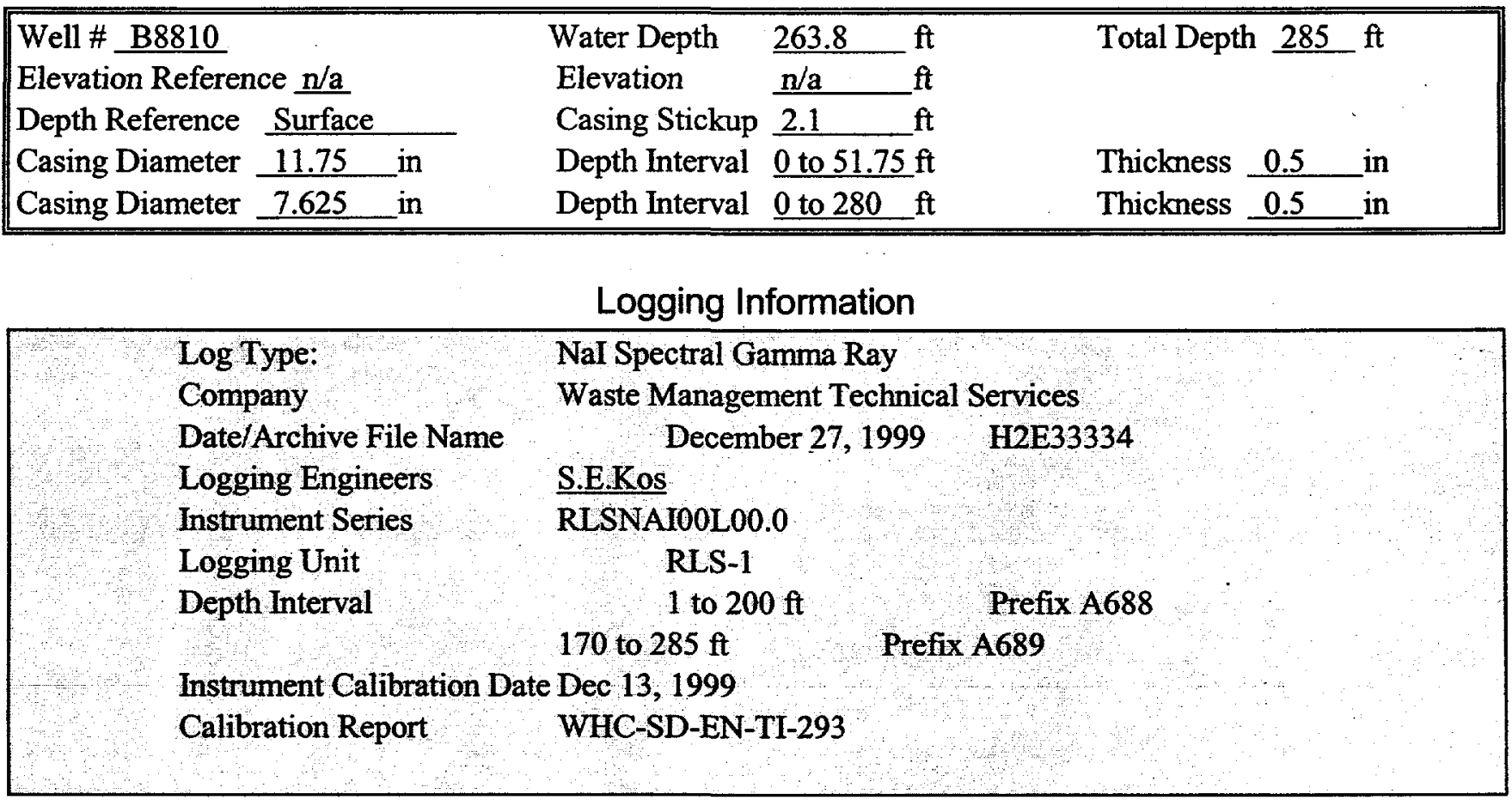

Analysis Information

Company

Analyst

Date

Depth Reference
Three Rivers Scientific

Russ Randall

February 15, 2000

Ground Surface

Notes Some individual spectra over 170 to 200 feet exhibited gain to high to measure the thorium photo peak. These spectra were deleted from the analysis. Since the deleted spectra were all contained in the repeated interval, no depth intervals were lost. 
Depth (feet)
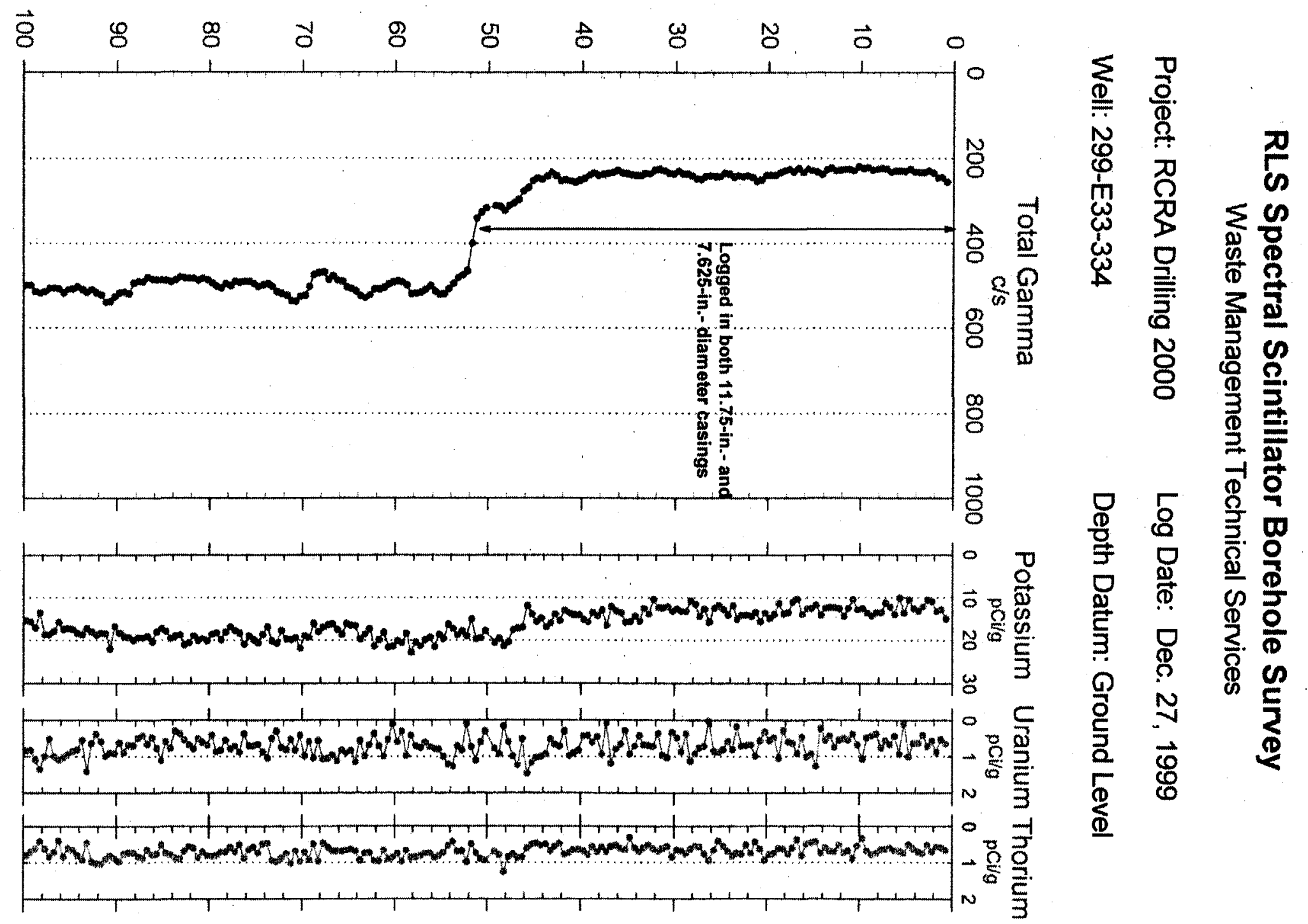


\section{RLS Spectral Scintillator Borehole Survey}

\section{Waste Management Technical Services}

Project: RCRA Drilling 2000

Well: 299-E33-334

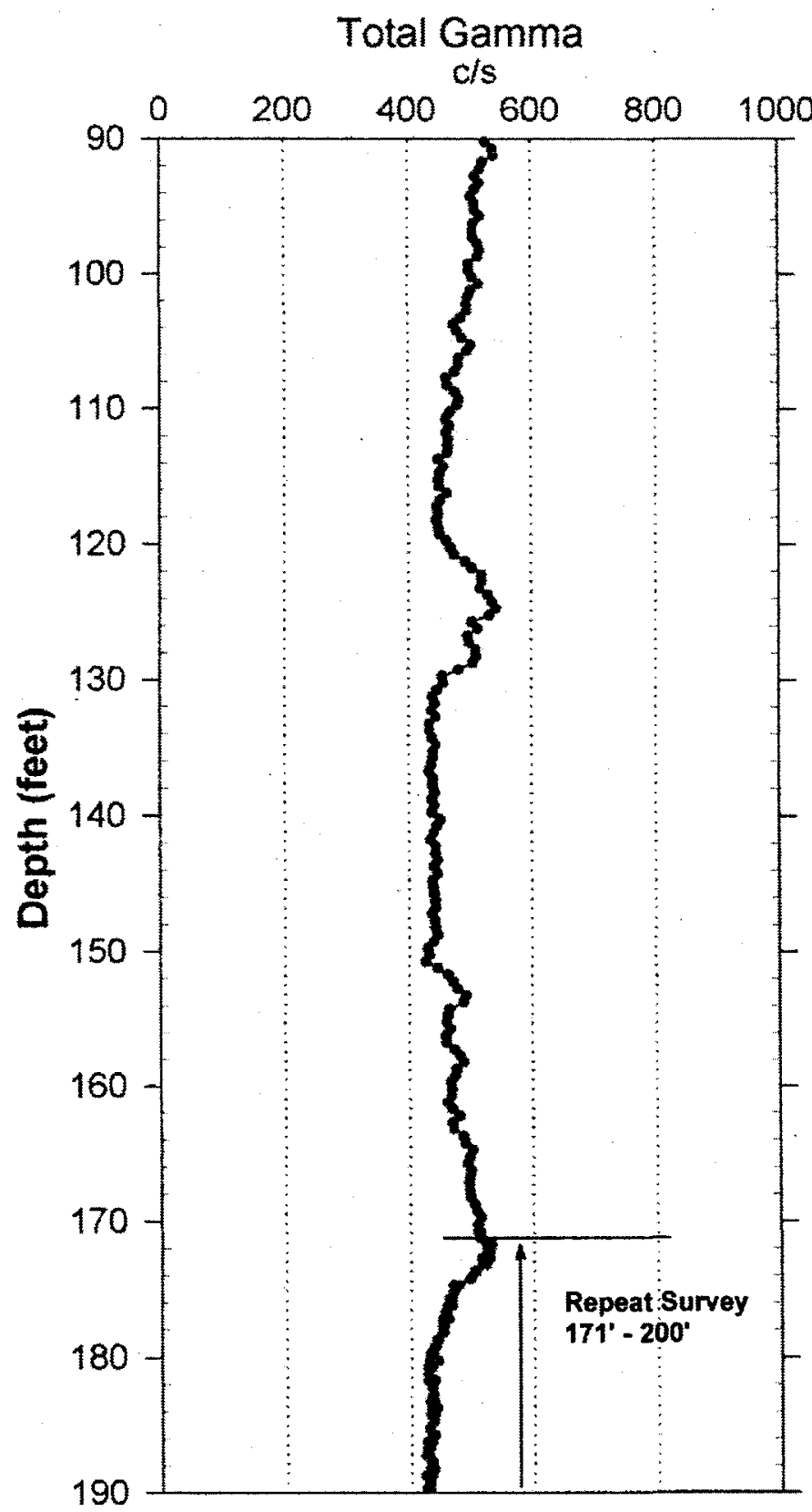

Log Date: Dec. 27, 1999

Depth Datum: Ground Level
Potassium Uranium Thorium $\mathrm{pCi} / \mathrm{g} \quad \mathrm{pCi} / \mathrm{g} \quad \mathrm{pCi} / \mathrm{g}$

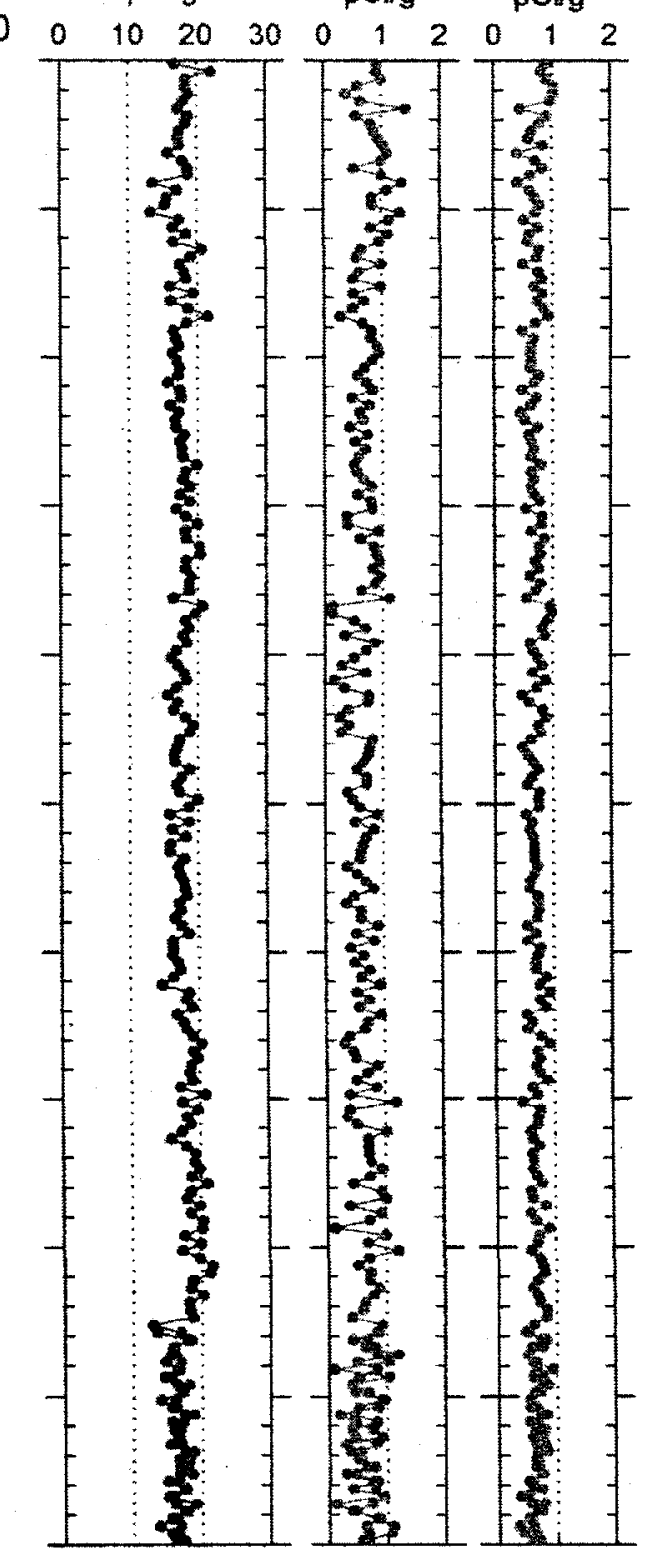

C. 4 


\section{RLS Spectral Scintillator Borehole Survey \\ Waste Management Technical Services}

Project: RCRA Drilling 2000

Well: 299-E33-334
Log Date: Dec. 27, 1999

Depth Datum: Ground Level

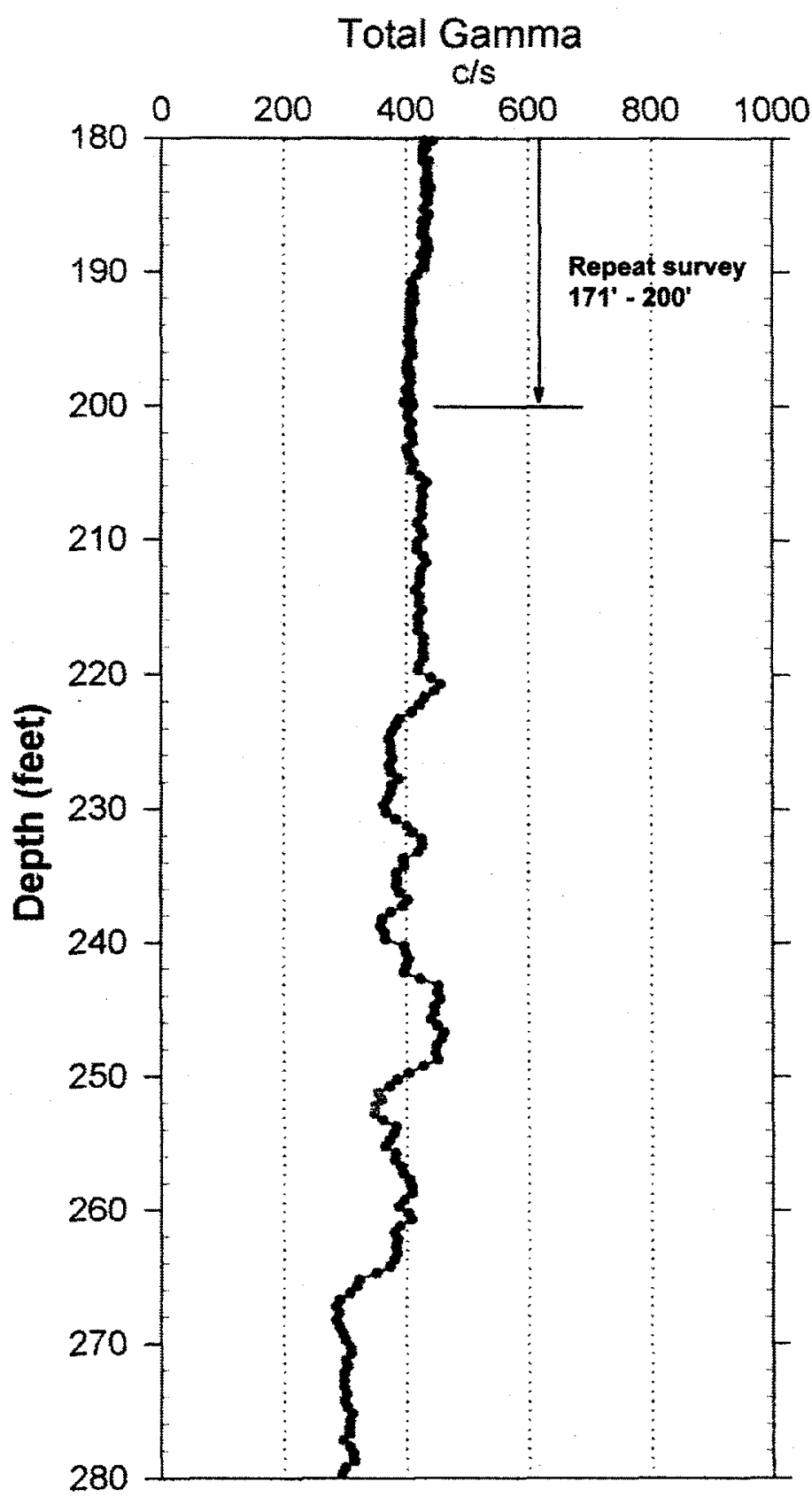

Potassium Uranium Thorium pCi/g pCilg pCig

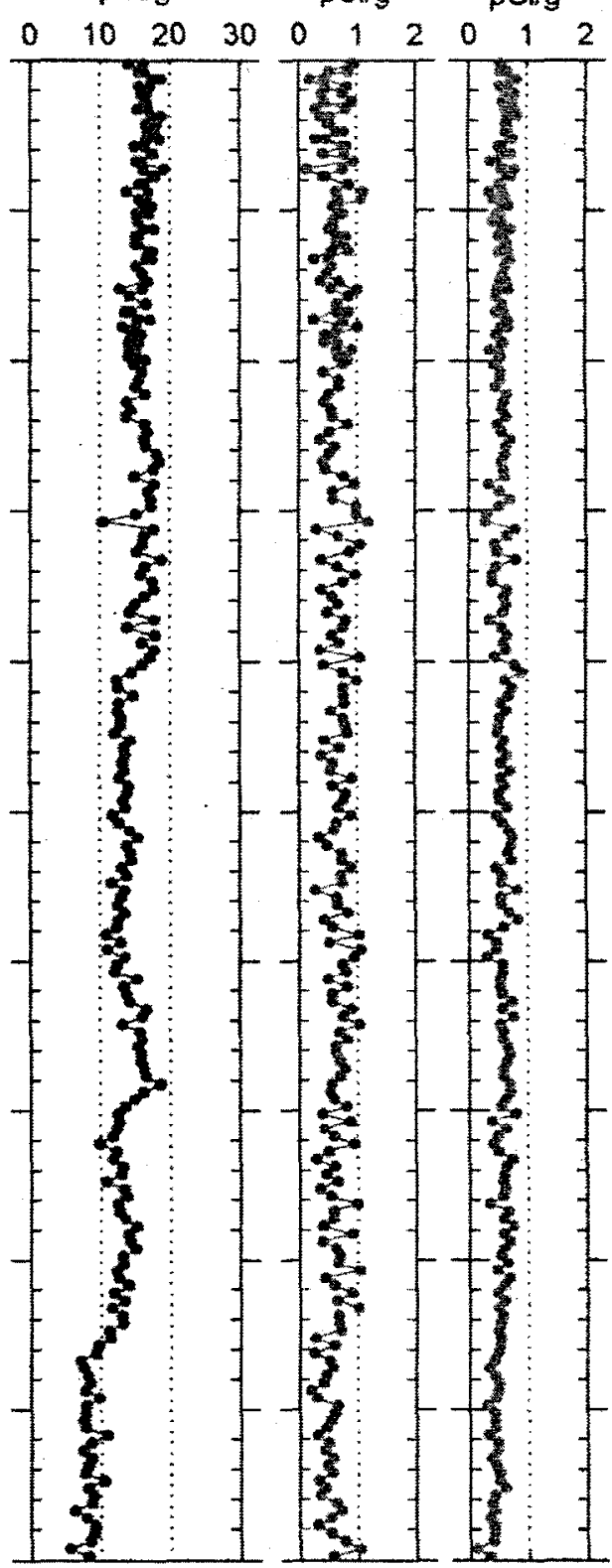


RLS Spectral Scintillator Borehole Survey

Waste Management Technical Services

Project: RCRA Drilling 2000

Well: 299-E33-334

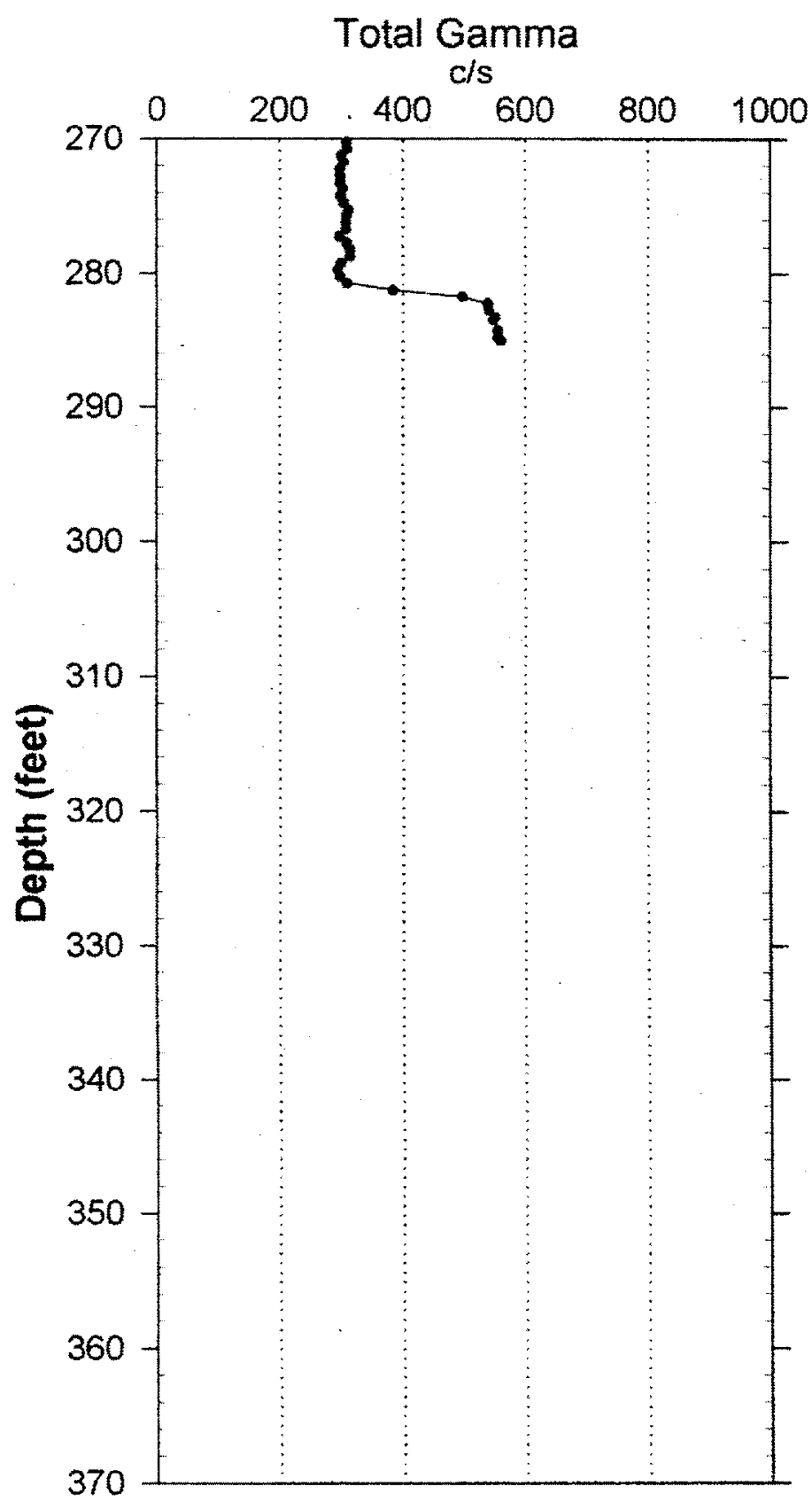

Log Date: Dec. 27, 1999

Depth Datum: Ground Level

Potassium Uranium Thorium $\mathrm{pCi} / \mathrm{g} \quad \mathrm{pCi} / \mathrm{g} \quad \mathrm{pCi} / \mathrm{g}$

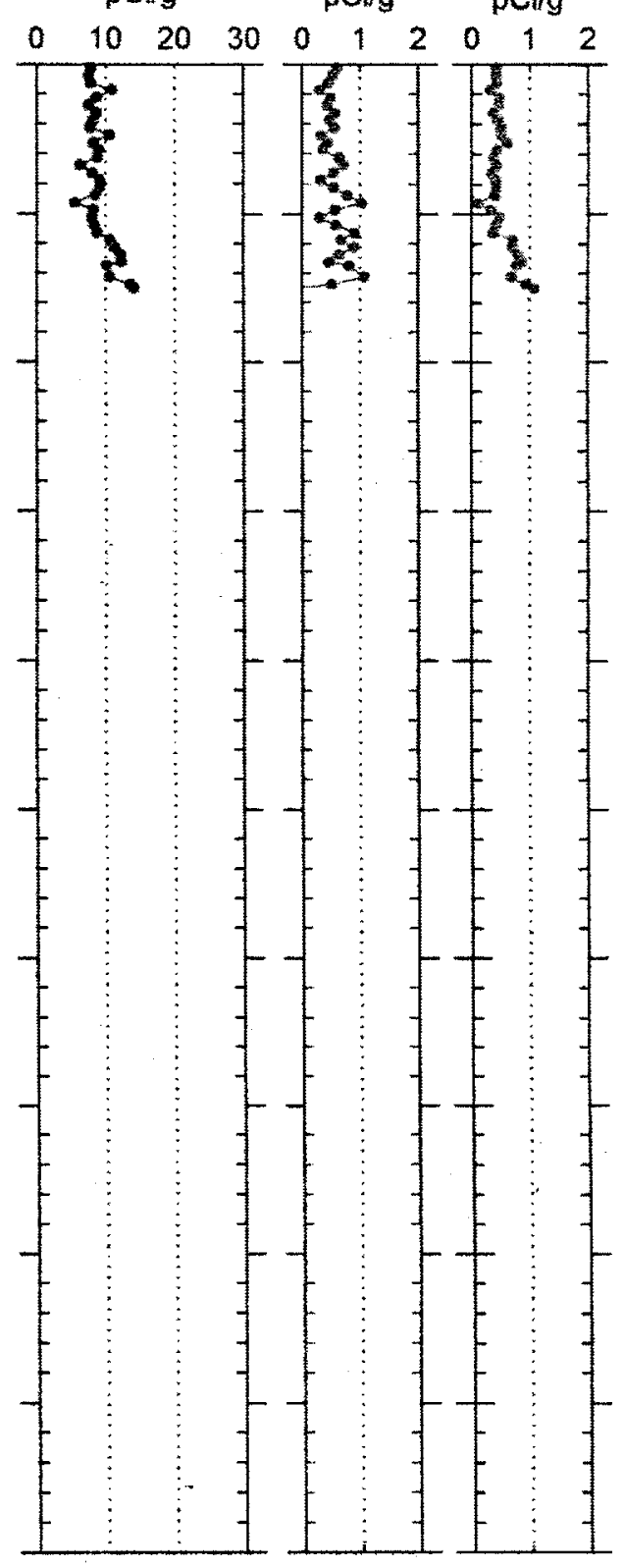




\section{RLS Scintillator Gamma Ray Borehole Survey \\ Waste Management Technical Services}

\section{Log Analysis Summary Report}

Project: PNNL RCRA

Log Type: NaI Spectral Gamma Ray
Well:

299-E33-334

Log Date: December 27, 1999

\section{General Notes:}

Total gamma is a response to geologic concentrations of natural radionuclides.

Log data collected with a depth reference of ground surface.

Log data over the depths from 170.75 to $1175.25,193.73$ to 194.25 , and 195.75 to 196.25 were lost due to excessive energy gain. Since these data points were covered in the repeat interval, all depths have at least one reading.

The energy to channel gain-settings changed throughout the logged intervals. The changes were outside of acceptable standards for the KUT computations. Thus a processing of the spectra to re-gain the energy using the potassium photo peak was performed. The results were successful except for the intervals listed above.

System Performance Verify: The pre- and post-log verification passed performance standards.

Repeat Interval: Based on the repeat interval, the logging system performed as per specifications.

Environmental Corrections: All radionuclide concentrations have been corrected for casing attenuation (entire well). Water correction was applied to depths deeper than 263.8 feet. No casing correction was applied to the total gamma due to Compton downscatter interference.

\section{Radionuclides:}

The potassium signal increases at 48 feet. The casing thickness changes from a total of 1.0 inch to 0.5 inch at 51.75 feet. Thus the increase in potassium at 48 feet is due to lithology and not an error in the casing thickness correction. 


\section{RLS Neutron-Neutron Moisture Survey \\ Waste Management Technical Services}

\section{LOG HEADER}

Project: RCRA drilling 1999

Well: 299-E33-334

Borehole Information

Well \# 299-E33-334

Elevation Reference $\mathrm{n} / \mathrm{a}$

Depth Reference Ground Surface

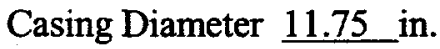

Casing Diameter $\underline{8.625}$ in.

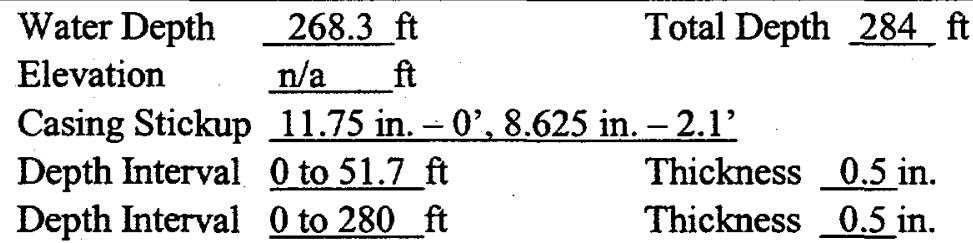

\section{Logging Information}

Log Type:

Company

Logging Engineers

Instrument Series

Logging Date

Logging Unit

Depth Interval
Neutron-Neutron Moisture

Waste Management Technical Services

S. E. Kos

RLSM00.0

December 27, 1999

RLS-1

$48.0^{\prime}$ to 168.0 '

$148.0^{\prime}$ to $264.0^{\prime}$

Prefix A690

A691

Instrument Calibration Date May 13, 1999

Calibration Report

WHC-SD-EN-TI-306, Rev. 0

Analysis Information

Company

Analyst

Date

Depth Reference

\section{Waste Management Technical Services}

Steven Kos

December 27, 1999

Ground Surface

Notes The moisture measurements were acquired at $0.250-\mathrm{ft}$ depth intervals at a logging speed of $1.0 \mathrm{ft}$ per minute. A repeat survey was conducted between depths of 148 and $168 \mathrm{ft}$. 


\section{Neutron-Neutron Moisture Survey \\ Waste Management Technical Services}

Project: 1999 RCRA Drilling Log Date : December 27, 1999 Borehole: 299-E33-334 Depth Datum: Ground Level

Moisture Vol. \%

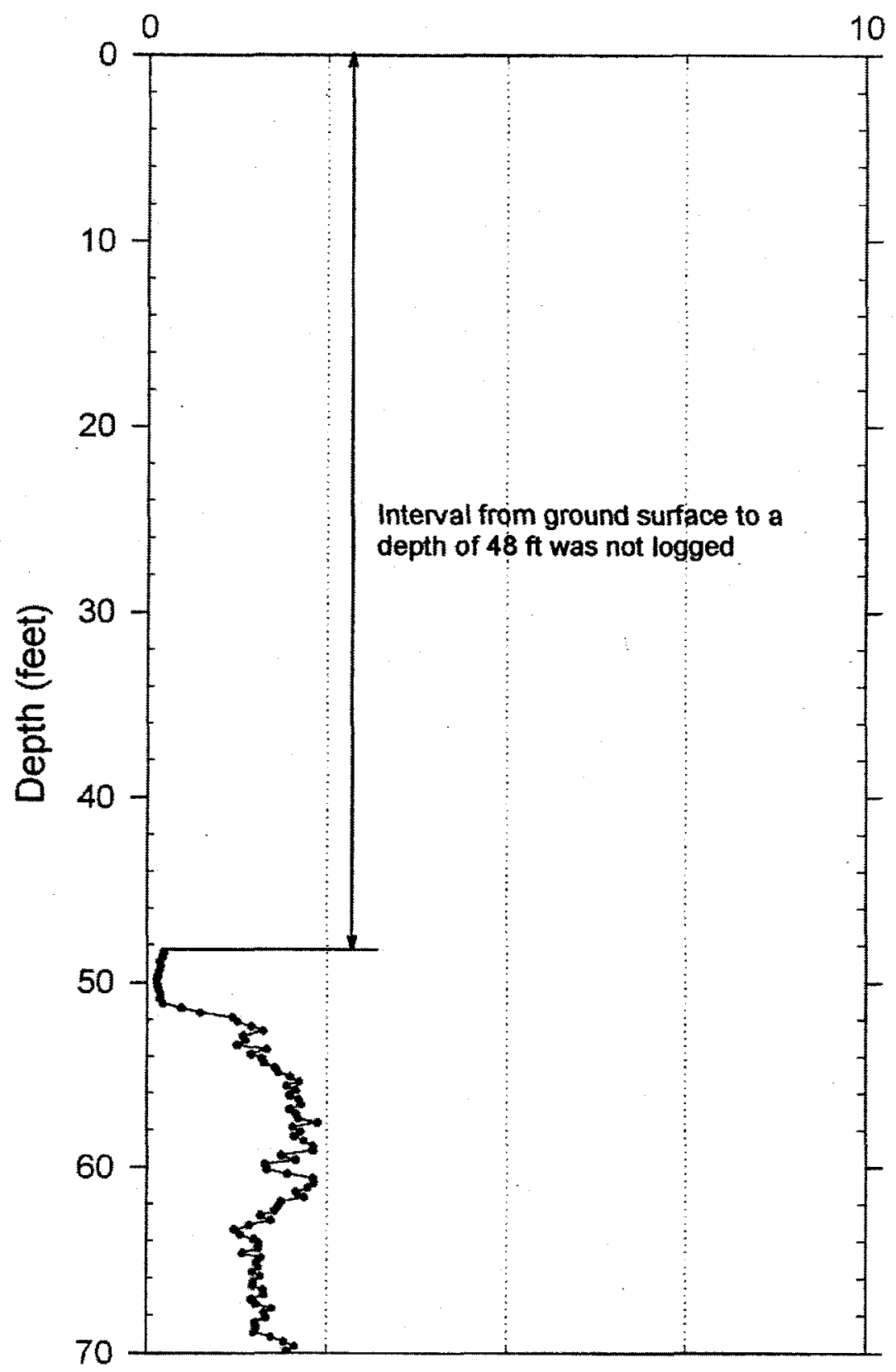

C.9 


\section{Neutron-Neutron Moisture Survey \\ Waste Management Technical Services}

Project: 1999 RCRA Drilling Log Date : December 27, 1999 Borehole: 299-E33-334 Depth Datum: Ground Level

Moisture Vol. \%

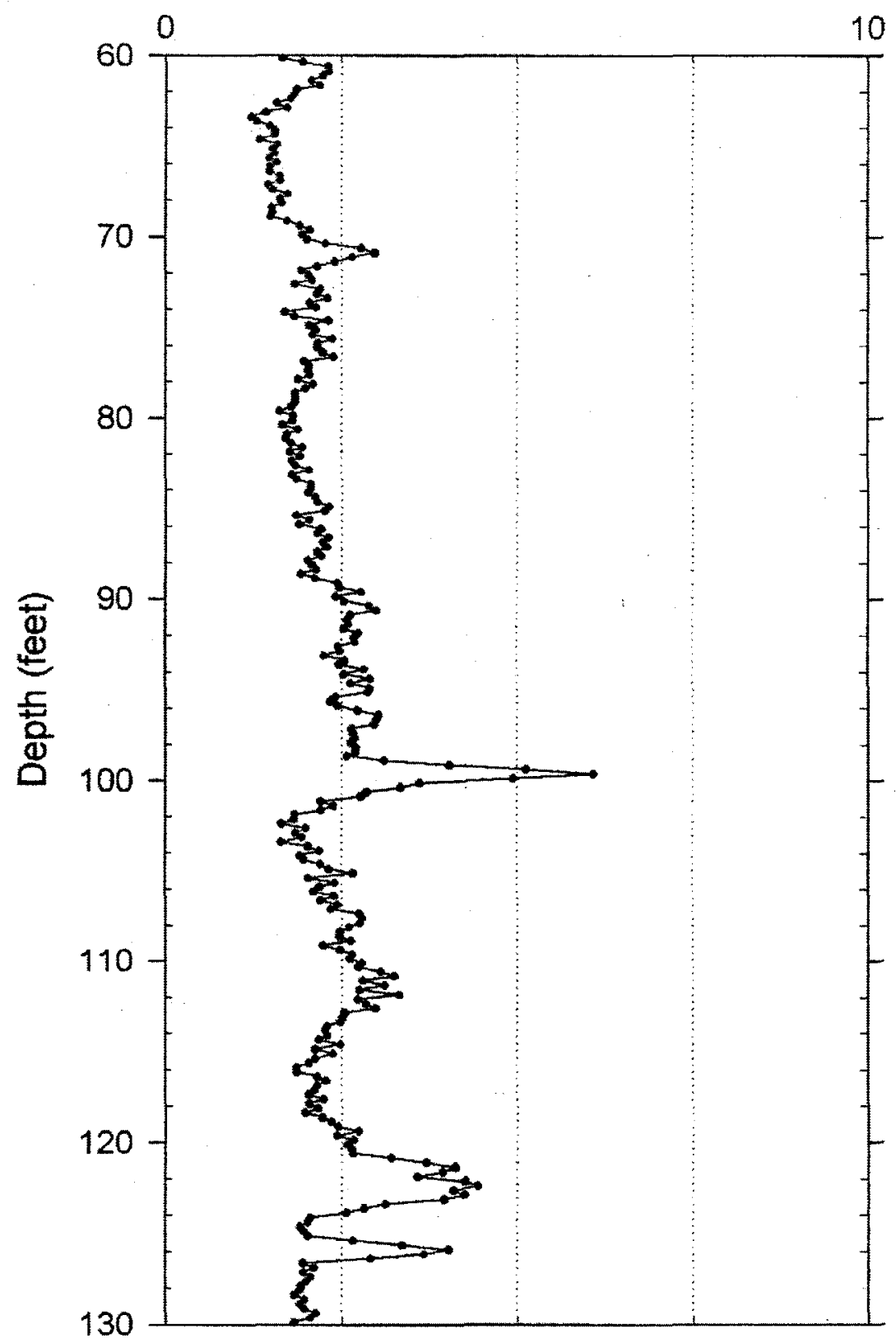

C. 10 


\section{Neutron-Neutron Moisture Survey \\ Waste Management Technical Services}

Project: 1999 RCRA Drilling Log Date : December 27, 1999 Borehole: 299-E33-334 Depth Datum: Ground Level

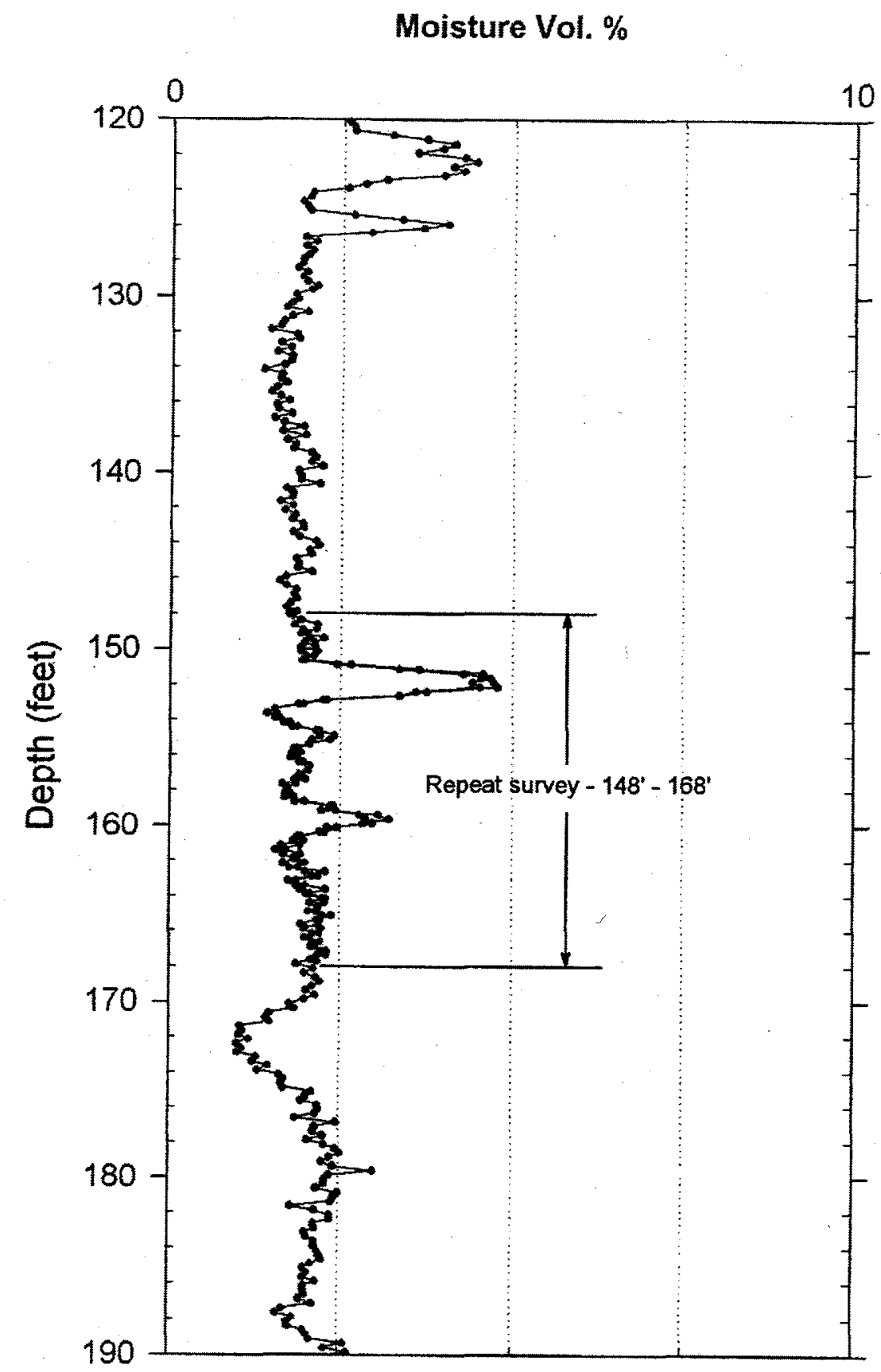




\section{Neutron-Neutron Moisture Survey \\ Waste Management Technical Services}

Project: 1999 RCRA Drilling Log Date : December 27, 1999 Borehole: 299-E33-334 Depth Datum: Ground Level

\section{Moisture Vol. \%}

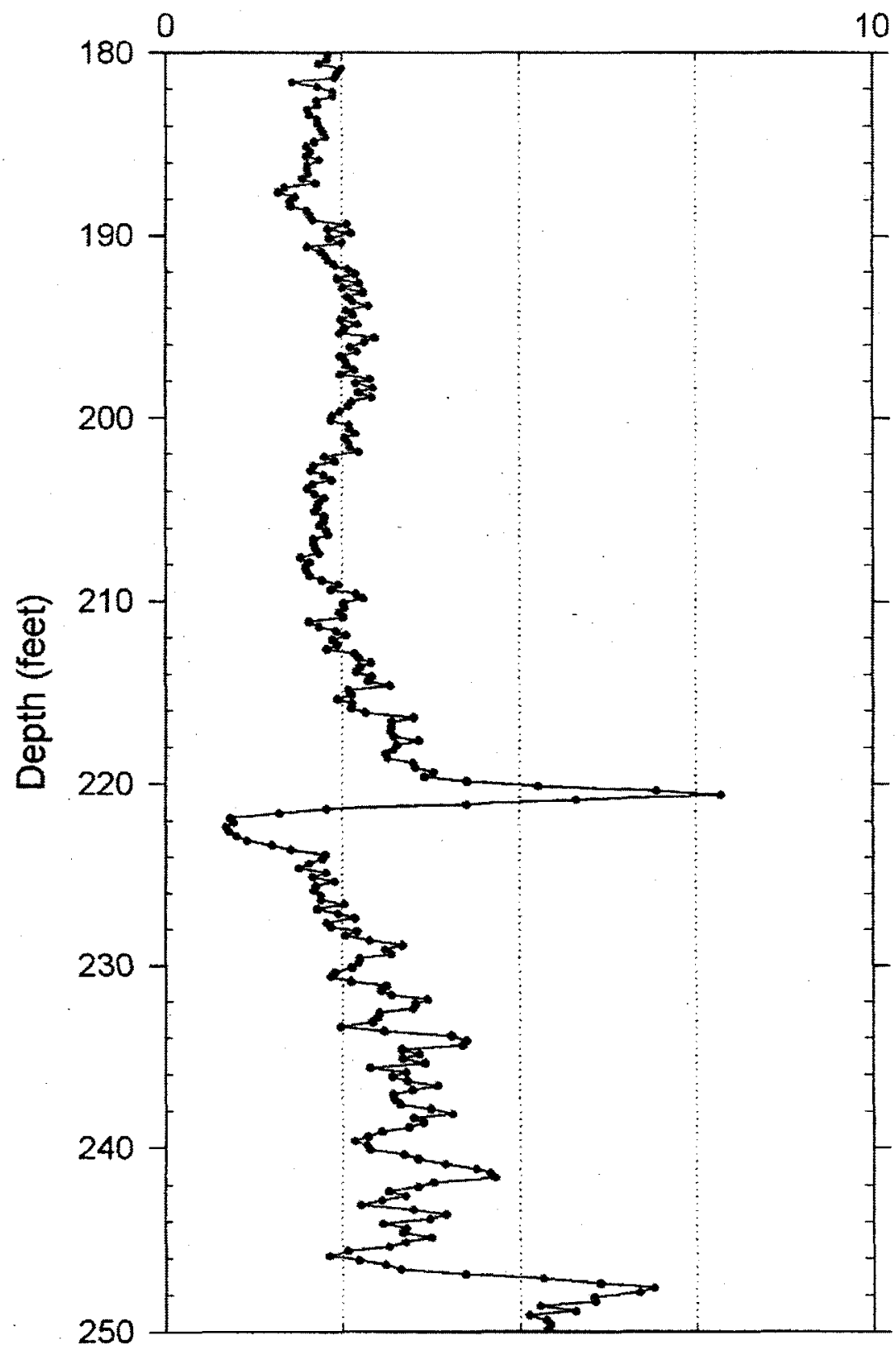

C. 12 


\section{Neutron-Neutron Moisture Survey \\ Waste Management Technical Services}

Project: 1999 RCRA Drilling Log Date : December 27, 1999 Borehole: 299-E33-334 Depth Datum: Ground Level

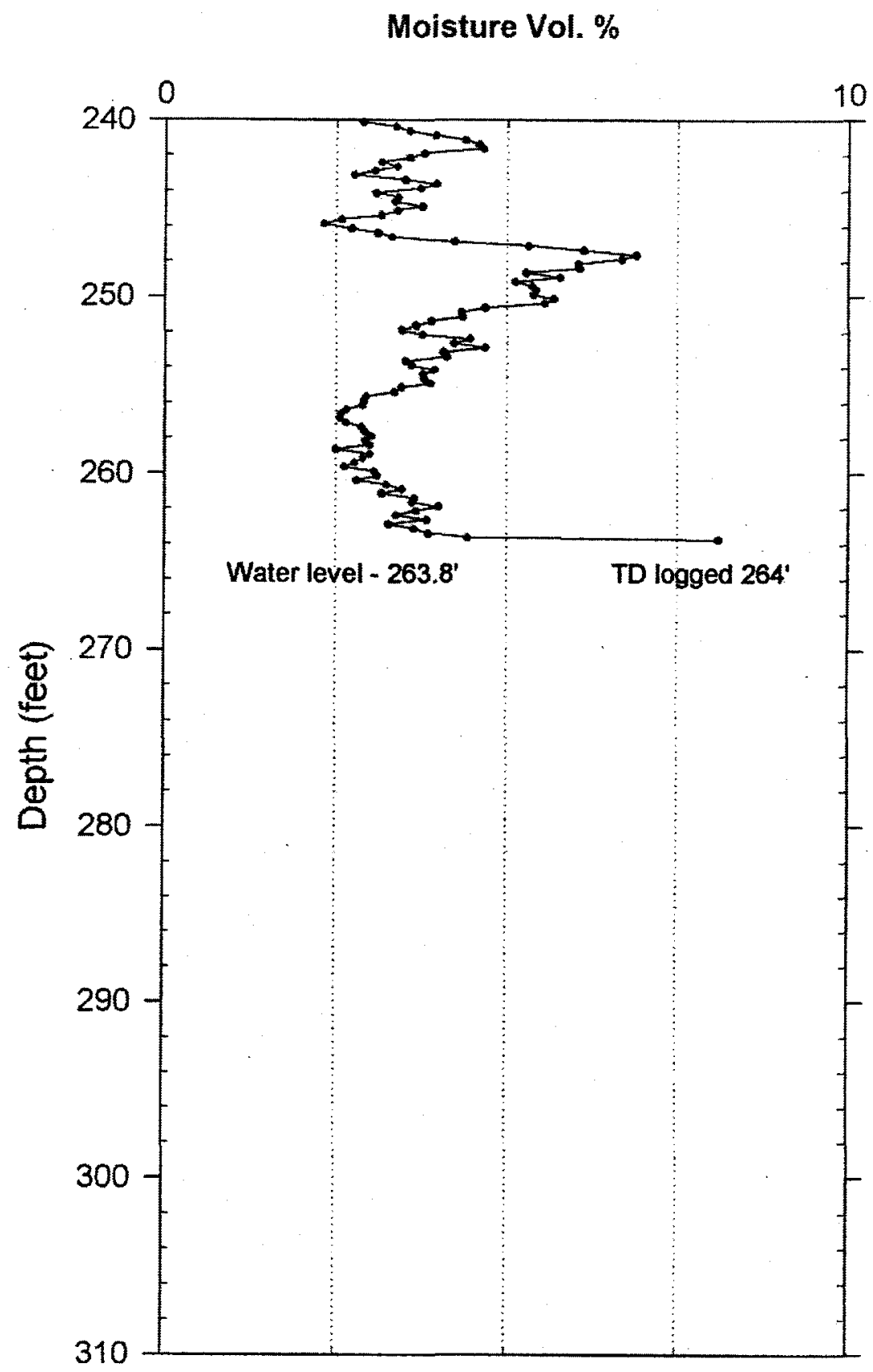

C. 13 


\title{
RLS Neutron-Neutron Moisture Survey \\ Waste Management Technical Services
}

\author{
Summary Report
}

Project: RCRA Drilling 1999

Well: $299-\mathrm{E} 33-334$

\section{General Notes}

All log data were collected with reference to ground surface. The moisture survey was not conducted in the 11.75-in.-diameter casing (from ground surface to a depth of $48 \mathrm{ft}$ ) since the logging tool is not calibrated for this size casing. The survey was terminated at a depth of $264 \mathrm{ft}$ where groundwater was encountered.

System Performance Verification: The pre- and post-survey verification passed performance standards, $-3.4 \%$ in the shield verifier.

Repeat Interval: A repeat survey was conducted between depths of 148 and $168 \mathrm{ft}$. The results show good repeatability of the moisture profiles from the original and repeat surveys.

Environmental Corrections: The moisture measurements have been corrected for casing attenuation throughout the entire well. A casing correction for 8-in.-diameter casing was applied to the data.

\section{Observations}

The moisture values are about 2 percent volumetric moisture content from a depth of about $50 \mathrm{ft}$, to a depth of $210 \mathrm{ft}$. At a depth of $210 \mathrm{ft}$, the moisture content fluctuates between slightly below 2 percent to slightly above 8 percent at a depth of $220 \mathrm{ft}$. The lowest moisture content values (other than within the short interval logged in the 11-in.- and 8-in.-diameter casings between depths of 48 and $51 \mathrm{ft}$ ) were measured at a depth of $222 \mathrm{ft}$. Several peaks of elevated moisture content (above the 2 percent background) occur at depths of $100 \mathrm{ft}, 122 \mathrm{ft}, 126 \mathrm{ft}, 152 \mathrm{ft}$, and $248 \mathrm{ft}$. The intervals of elevated moisture content most likely correlate to occurrence of fine- grained sediments that retain moisture.

The measurements acquired in double casings between depths of 48 and $51 \mathrm{ft}$ and are not valid measurements. These measurements can be utilized to determine the bottom of the double casing string, which is located at a depth of $51 \mathrm{ft}$.

The moisture content increases (to an off-scale value) at a depth of about $264 \mathrm{ft}$ where groundwater is encountered. 


\section{RLS Scintillator Gamma Ray Borehole Survey \\ Waste Management Technical Services}

\section{Log Header}

Project: PNNL RCRA

Well: 299-E33-335

\section{Log Type: Nal Spectral Gamma Ray}

Borehole Information

\begin{tabular}{|c|c|c|c|c|c|}
\hline Well \# B8811 & Water Depth & $\underline{278}$ & Total Depth & h $\underline{28}$ & \\
\hline Elevation Reference $\underline{\mathbf{n} / \mathbf{a}}$ & Elevation & $\underline{n} / \mathrm{a}$ & & & \\
\hline Depth Reference Surface & Casing Stickup & .25 & & & \\
\hline Casing Diameter 11.75 in & Depth Interval & 0 to 50 & Thickness & 0.5 & \\
\hline Casing Diameter 7.625 in & Depth Interval & 0 to $280 \mathrm{ft}$ & Thickness & 0.5 & - \\
\hline
\end{tabular}

Logging Information

Log Type:
Company
Date/Archive File Name
Logging Engineers
Instrument Series
Logging Unit
Depth Interval

Depth Interval
NaI Spectral Gamma Ray

Waste Management Technical Services

February 2, 2000

H2E33335

J.E.Meisner

RLSN2.0

RLS-1

0 to $175 \mathrm{ft}$

170 to $282 \mathrm{ft}$

Instrument Calibration Date Feb 18, 1999

Calibration Report

WHC-SD-EN-TI-293
Prefix A697

Prefix A698

\section{Analysis Information}

Company

Analyst

Date

Depth Reference
Three Rivers Scientific

Russ Randall

February 15, 2000

Ground Surface

Notes None. 


\section{RLS Spectral Scintillator Borehole Survey Waste Management Technical Services}

Project: RCRA Drilling $2000 \quad$ Log Date: Feb. 2, 2000

Well: 299-E33-335

Depth Datum: Ground Level

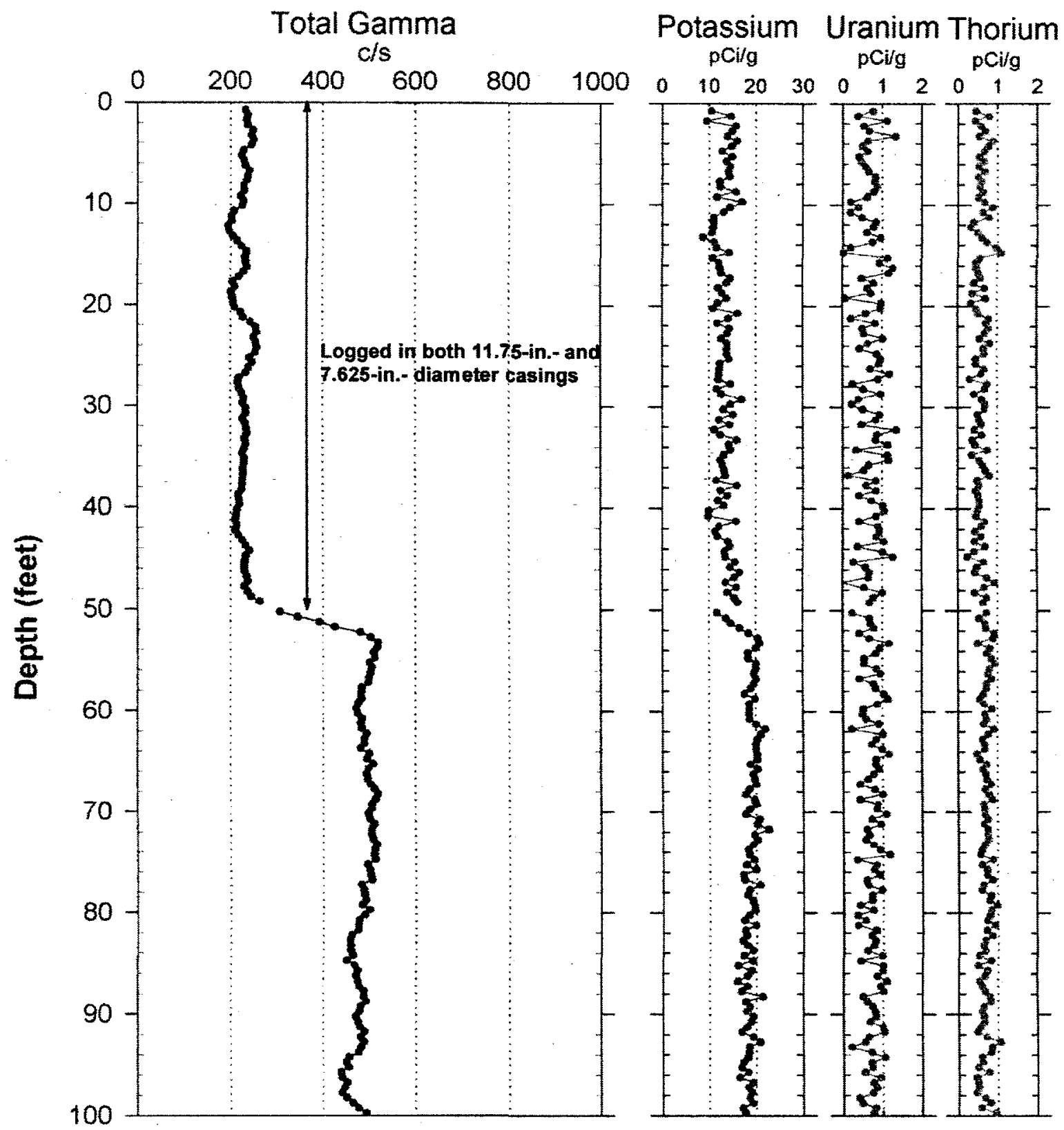




\section{RLS Spectral Scintillator Borehole Survey Waste Management Technical Services}

Project: RCRA Drilling 2000

Well: 299-E33-335
Log Date: Feb. 2, 2000

Depth Datum: Ground Level

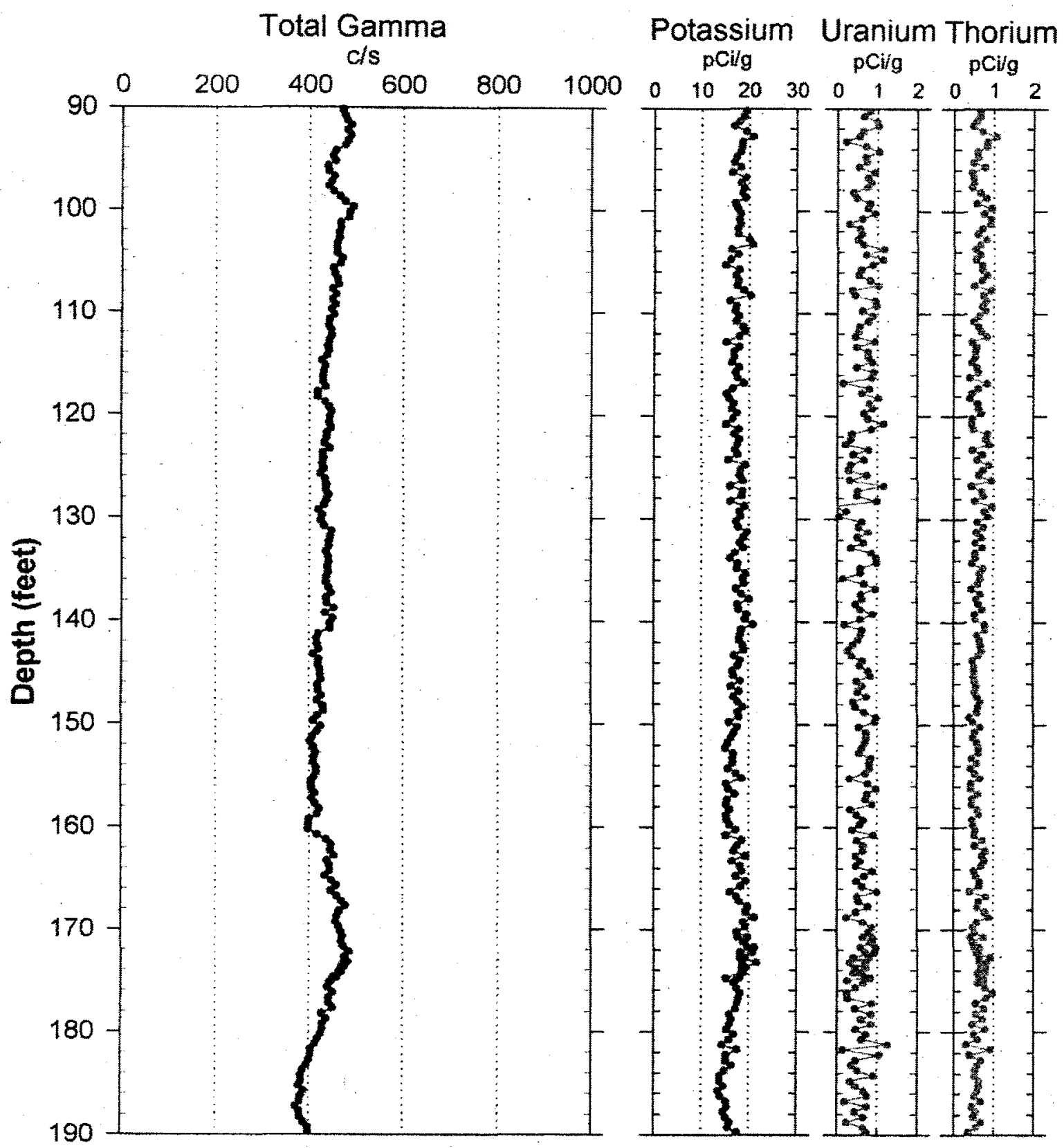




\section{RLS Spectral Scintillator Borehole Survey Waste Management Technical Services}

Project: RCRA Drilling 2000

Well: 299-E33-335
Log Date: Feb. 2, 2000

Depth Datum: Ground Level

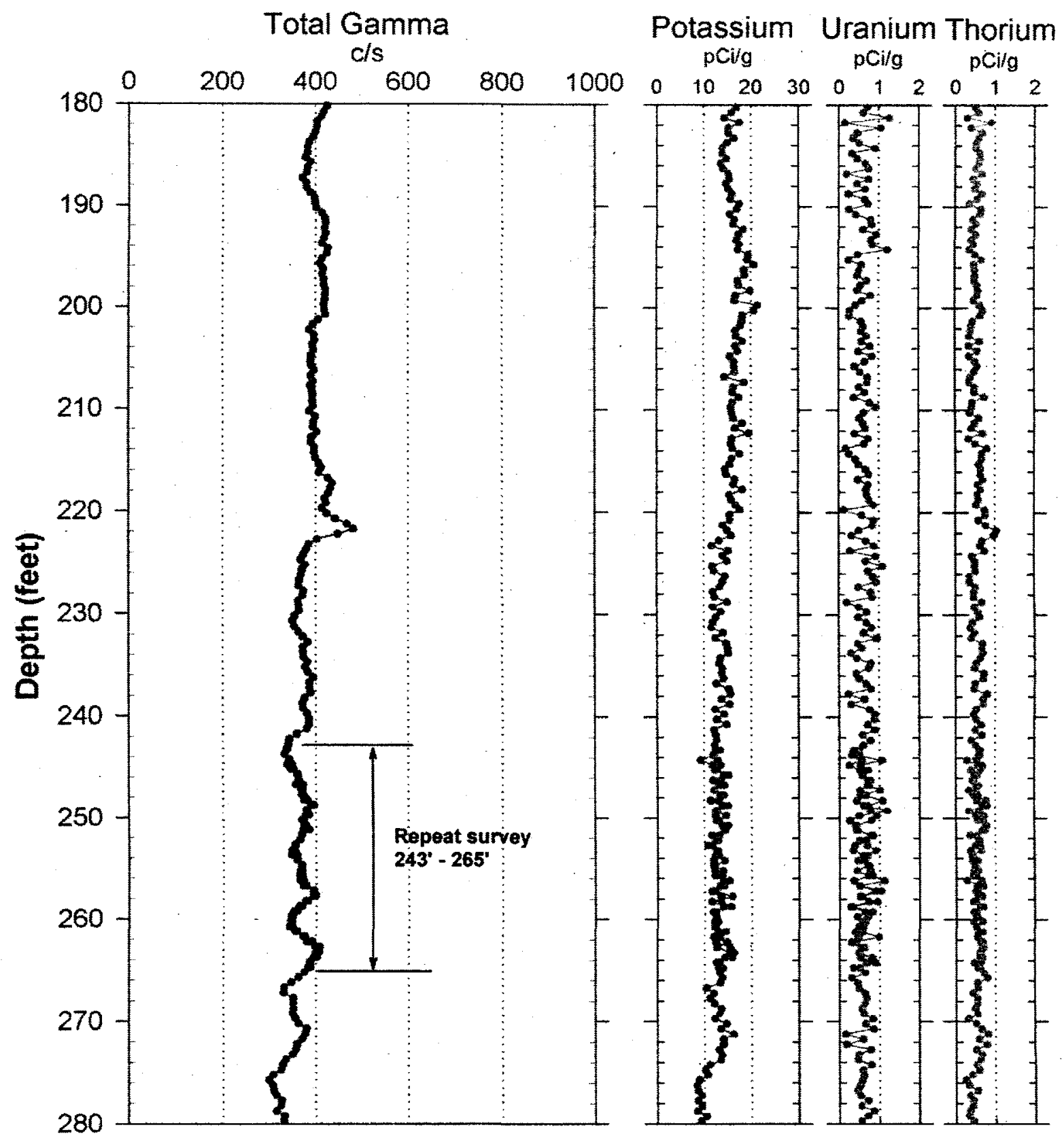

C. 18 
RLS Spectral Scintillator Borehole Survey Waste Management Technical Services

Project: RCRA Drilling 2000

Well: 299-E33-335

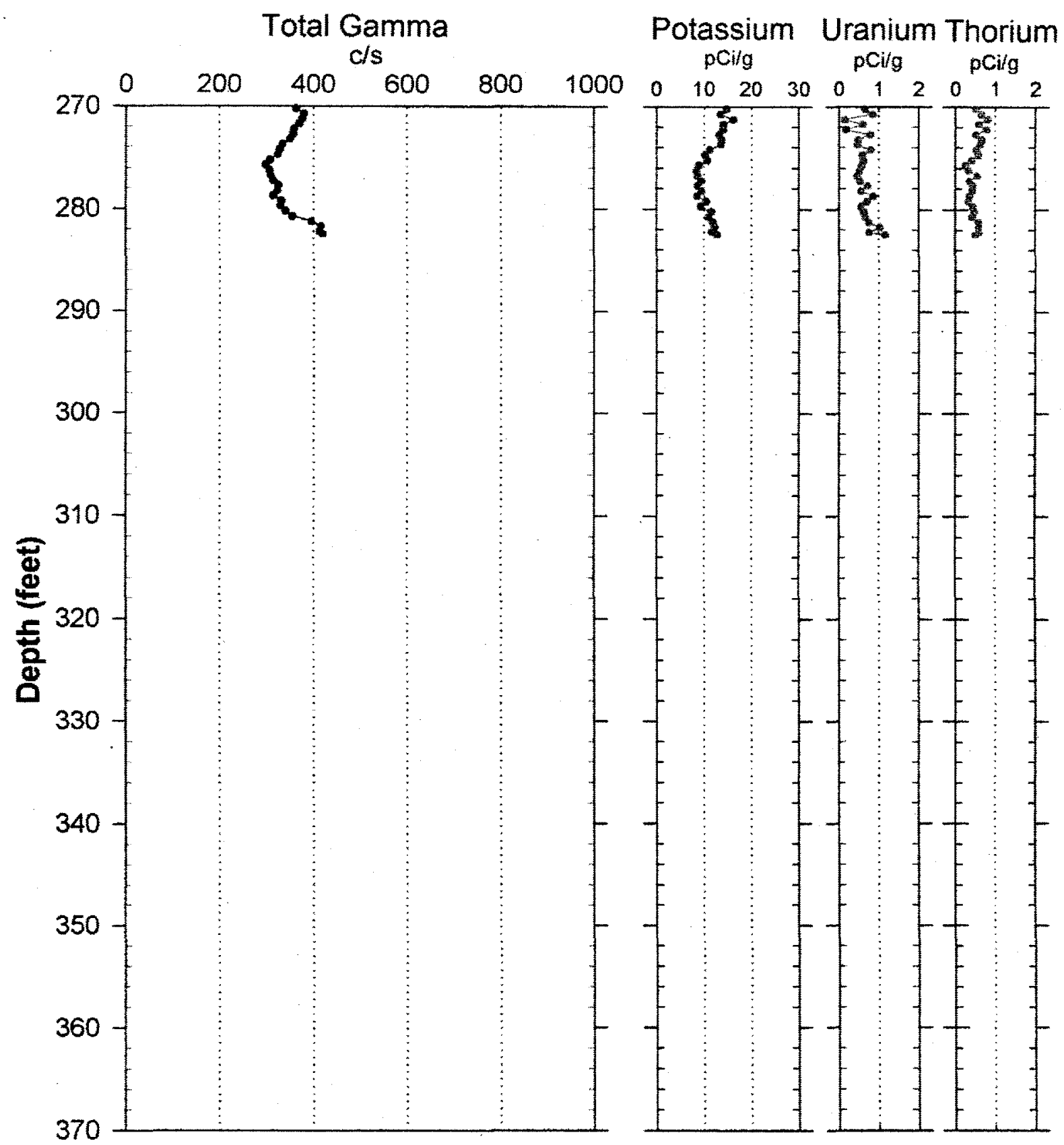

Log Date: Feb. 2, 2000

Depth Datum: Ground Level

Potassium Uranium Thorium 


\section{RLS Scintillator Gamma Ray Borehole Survey}

Waste Management Technical Services

\section{Log Analysis Summary Report}

Project: PNNL RCRA

Well:

299-E33-335

Log Type: NaI Spectral Gamma Ray

Log Date: February 2, 2000

\section{General Notes:}

Total gamma is a response to geologic concentrations of natural radionuclides.

Log data collected with a depth reference of ground surface.

The energy to channel gain-settings changed very little throughout the logged intervals. A processing of the spectra to re-gain the energy using the potassium photo peak was performed. The results were successful.

System Performance Verify: The pre- and post-log verification passed performance standards.

Repeat Interval: Based on the repeat interval, the logging system performed as per specifications.

Environmental Corrections: All radionuclide concentrations have been corrected for casing attenuation (entire well). Water correction was applied to depths deeper than 278 feet. No casing correction was applied to the total gamma due to Compton downscatter interference.

\section{Radionuclides:}

The potassium signal increases at 50 feet. The casing thickness changes from a total of 1.0 inch to 0.5 inch at 50 feet. However, the increase in potassium at 50 feet is due to lithology and not an error in the casing thickness correction. 


\section{RLS Neutron-Neutron Moisture Survey \\ Waste Management Technical Services}

\section{LOG HEADER}

Project: RCRA drilling 1999

Well: $299-E 33-335$

Borehole Information

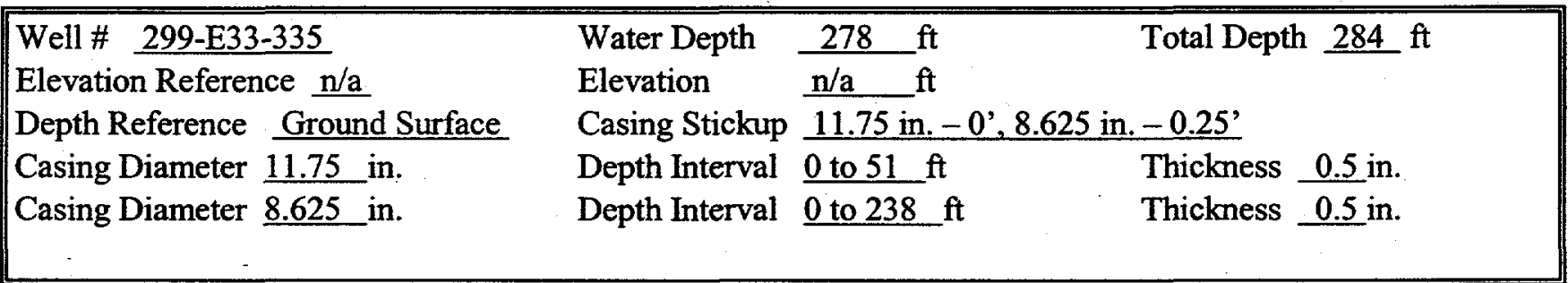

Logging Information

\begin{tabular}{|c|c|}
\hline Log Type: & Neutron-Neutron Moisture \\
\hline Company & Waste Management Technical Services \\
\hline Logging Engineers & J.E. Meisner \\
\hline Instrument Series & RLSM00.0 \\
\hline Logging Date & February 3, 2000 \\
\hline Logging Unit & RLS-1 \\
\hline \multirow[t]{3}{*}{ Depth Interval } & $45.0^{\prime}$ to $165.0^{\prime}$ \\
\hline & $162.0^{\prime}$ to $215.0^{\prime}$ \\
\hline & $80.0^{\prime}$ to $95.0^{\prime}$ \\
\hline \multicolumn{2}{|c|}{ Instrument Calibration Date May 13, 1999} \\
\hline \multirow[t]{2}{*}{ Calibration Report } & WHC-SD-EN-TI-306, Rev. 0 \\
\hline & Analysis Information \\
\hline Company & Waste Management Technical Services \\
\hline Analyst & Steven Kos \\
\hline Date & March 13, 2000 \\
\hline Depth Reference & Ground Surface \\
\hline
\end{tabular}

Notes The moisture measurements were acquired at $0.250-\mathrm{ft}$ depth intervals at a logging speed of $1.0 \mathrm{ft}$ per minute. Repeat surveys were conducted between depths of 80 and $95 \mathrm{ft}$, between depths of 147 and 150 $\mathrm{ft}$, between depths of 162 and $165 \mathrm{ft}$, and between depths of 215 and $225 \mathrm{ft}$. 


\section{Neutron-Neutron Moisture Survey}

Waste Management Technical Services

Project: 1999 RCRA Drilling Log Date : February 3, 2000

Borehole: 299-E33-335 Depth Datum: Ground Level

Moisture Vol. \%

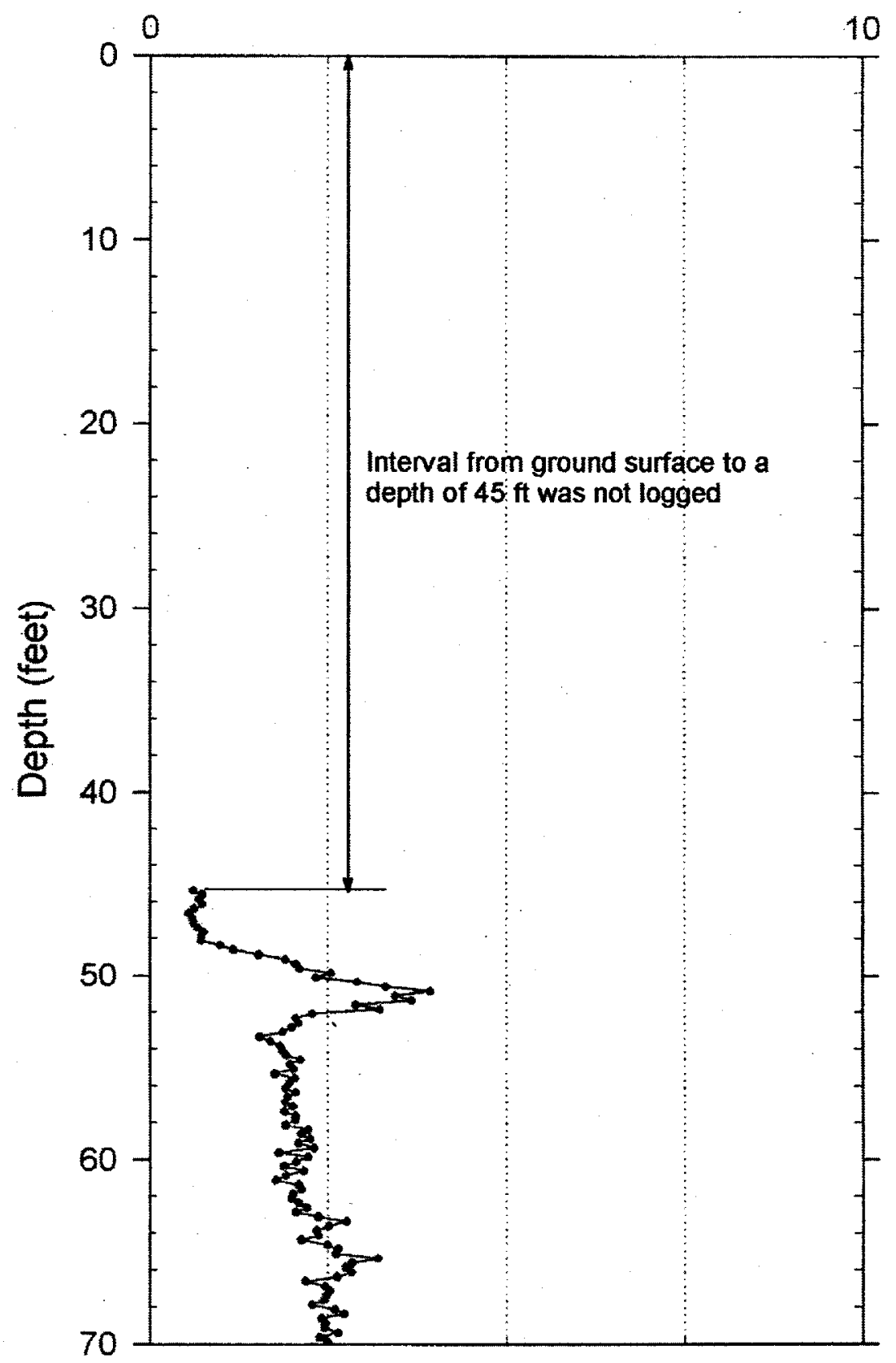




\section{Neutron-Neutron Moisture Survey \\ Waste Management Technical Services}

Project: 1999 RCRA Drilling Log Date : February 3, 2000 Borehole: 299-E33-335 Depth Datum: Ground Level

Moisture Vol. \%

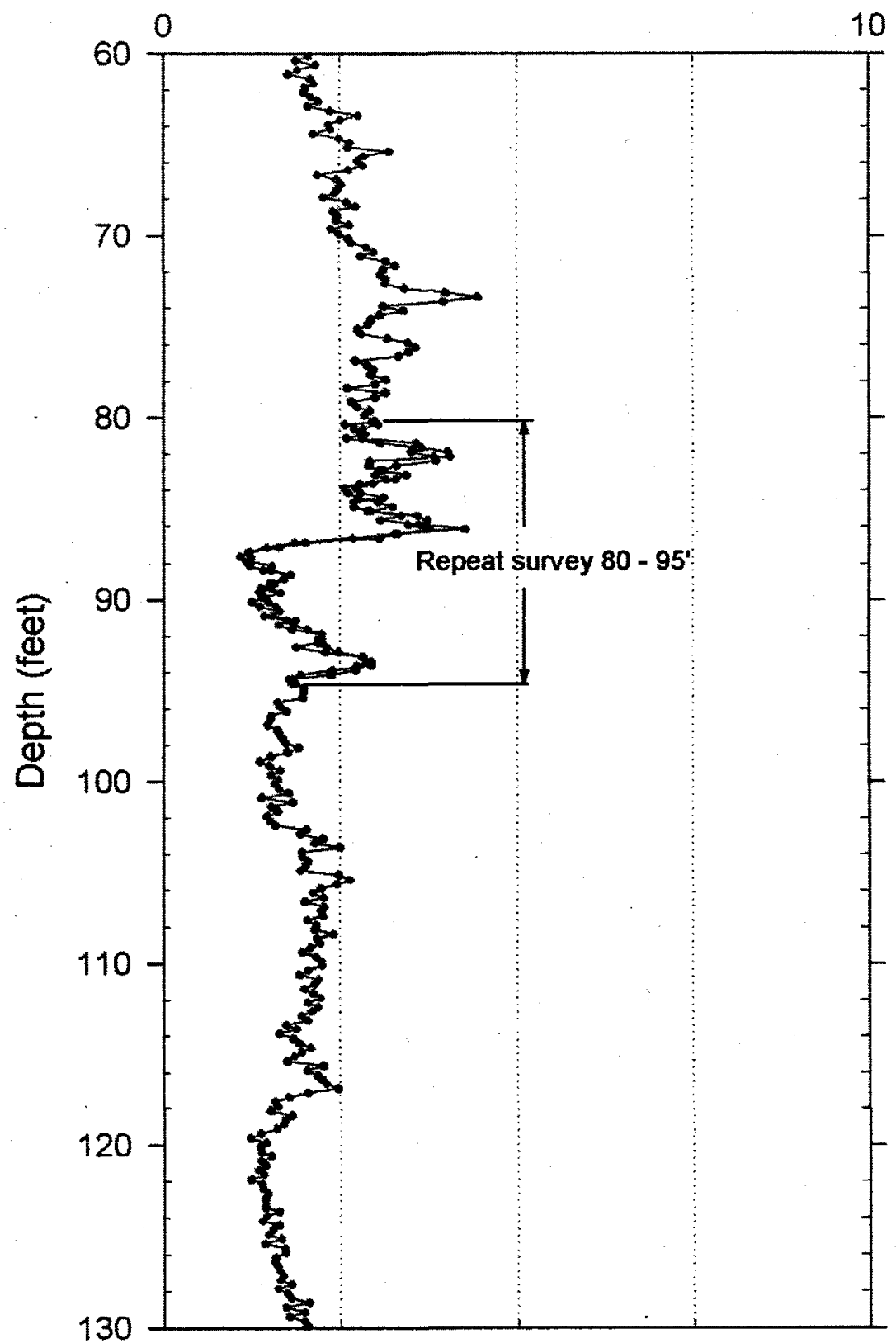




\section{Neutron-Neutron Moisture Survey}

Waste Management Technical Services

Project: 1999 RCRA Drilling Log Date : February 3, 2000 Borehole: 299-E33-335 Depth Datum: Ground Level

Moisture Vol. \%

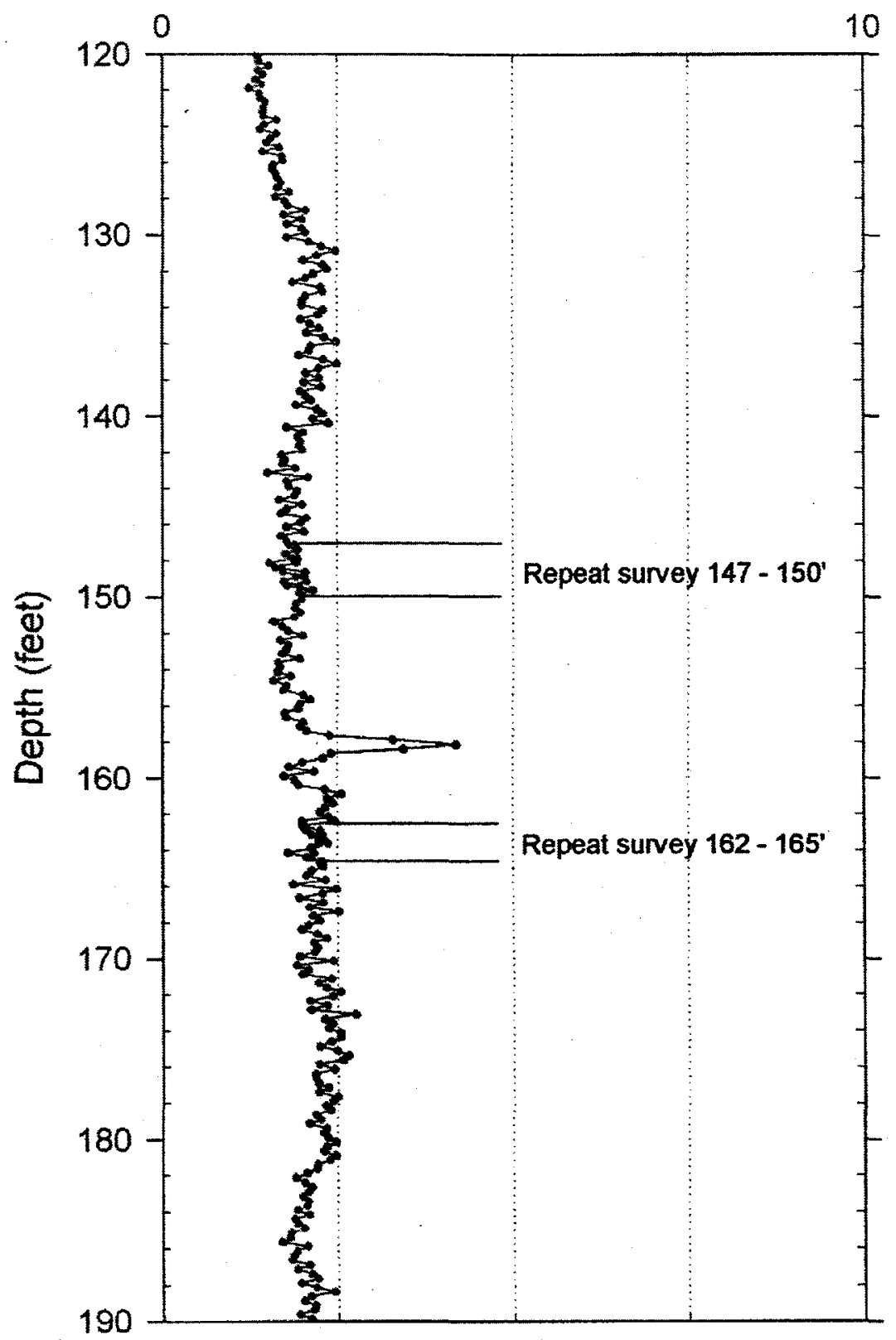




\section{Neutron-Neutron Moisture Survey \\ Waste Management Technical Services}

Project: 1999 RCRA Drilling Log Date : February 3, 2000 Borehole: 299-E33-335 Depth Datum: Ground Level

Moisture Vol. \%

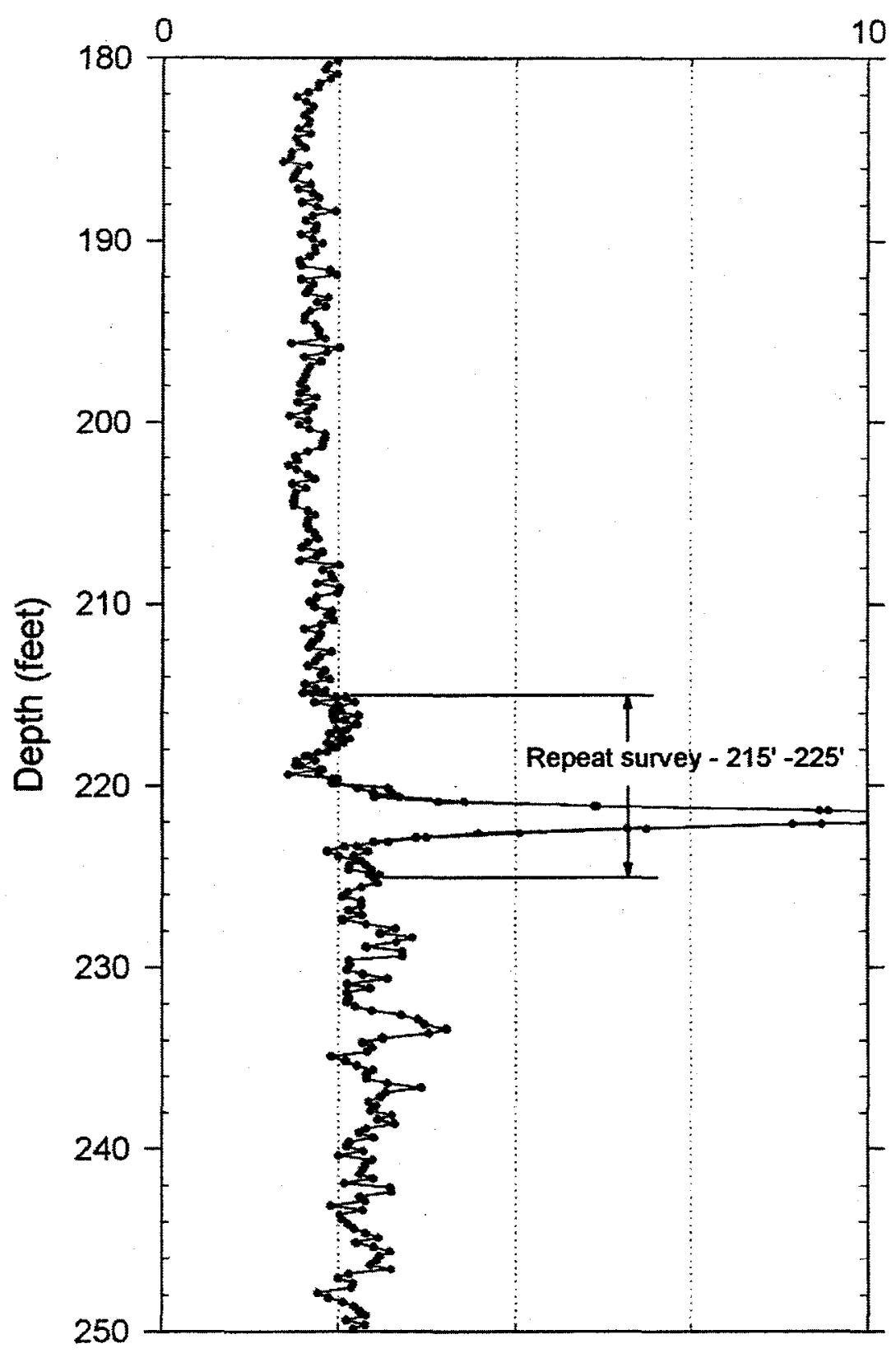

C. 25 


\section{Neutron-Neutron Moisture Survey}

Waste Management Technical Services

Project: 1999 RCRA Drilling Log Date : February 3, 2000 Borehole: 299-E33-335 Depth Datum: Ground Level

Moisture Vol. \%

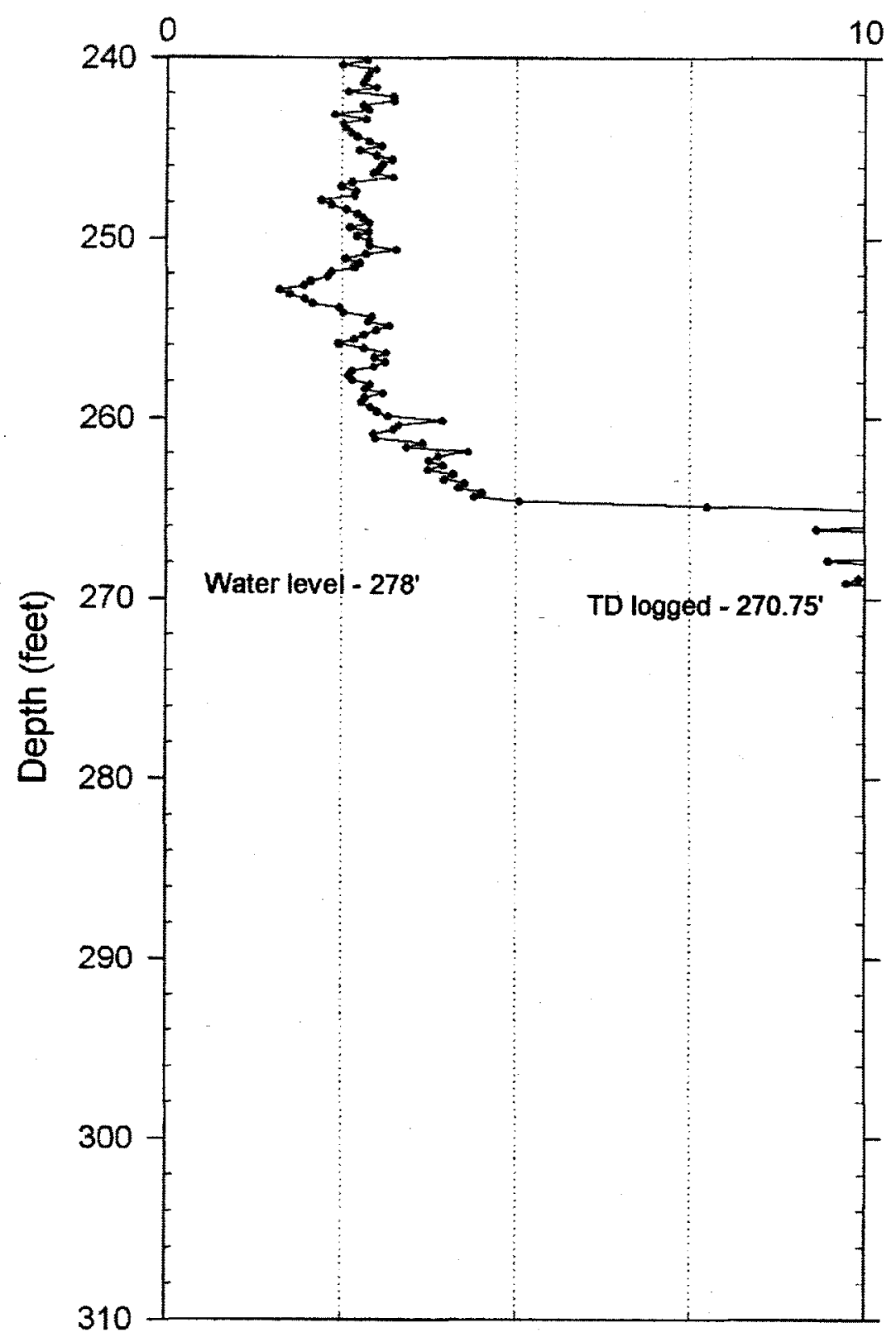




\section{RLS Neutron-Neutron Moisture Survey \\ Waste Management Technical Services}

Summary Report

Project: RCRA Drilling 1999

Well: 299-E33-335

\section{General Notes}

All log data were collected with reference to ground surface. The moisture survey was not conducted in the 11.75-in.-diameter casing (from ground surface to a depth of $45 \mathrm{ft}$ ) since the logging tool is not calibrated for this size casing. The survey was terminated at a depth of $270.75 \mathrm{ft}$ where groundwater was encountered.

System Performance Verification: The pre- and post-survey verification passed performance standards, $-3.4 \%$ in the shield verifier.

Repeat Interval: Repeat surveys were conducted between depths of 80 and $95 \mathrm{ft}$, between depths of 147 and $150 \mathrm{ft}$, between depths of 162 and $165 \mathrm{ft}$, and between depths of 215 and $225 \mathrm{ft}$. The results show good repeatability of the moisture profiles from the original and repeat surveys.

Environmental Corrections: The moisture measurements have been corrected for casing attenuation throughout the entire well. A casing correction for 8-in.-diameter casing was applied to the data.

\section{Observations}

The moisture values are slightly less than 2 percent volumetric moisture content throughout most of the log. The values increase slightly in the interval between depths of about 70 and $87 \mathrm{ft}$. Within this interval the maximum value detected in this boreholes of about 4 percent moisture content was measured (at a depth of $86 \mathrm{ft}$ ). Over the short interval between depths of 50 and $52 \mathrm{ft}$, the moisture content values reach a high of about 4 percent.

Over the interval between 45 and about $48 \mathrm{ft}$, the moisture measurements are not valid because they were acquired in two casings. The do indicate that the bottom of the double casing string is located at a depth of about $48 \mathrm{ft}$.

The moisture content increases (to an off-scale value) at a depth of about $215 \mathrm{ft}$ where groundwater is encountered. 
Appendix D

Groundwater Chemistry Data 


\section{Appendix D}

\section{Groundwater Chemistry Data}

This appendix contains analytical results from groundwater samples collected at $279 \mathrm{ft}$ below ground surface (bgs) from well 299-E33-334 and at $272 \mathrm{ft} \mathrm{bgs} \mathrm{from} \mathrm{well} \mathrm{299-E33-335} \mathrm{after} \mathrm{well} \mathrm{development.}$ Columns 6 and 7 in this appendix contain qualifiers that should be considered when using the analytical values. The definitions of the qualifiers are given below.
Qualifier
Definition
$\mathrm{U}$
Undetected at the detection limit.
C For inorganics - blank contamination is above the practical quantitation limit.
B For organics - compound was found in the blank (blank contamination).
For inorganics - result is less than the practical quantitation limit.
D Adjusted dilution factor. 
Table D.1. Composition of Groundwater from Wells 299-E33-334 and 299-E33-335

\begin{tabular}{|c|c|c|c|c|c|c|c|}
\hline Constituent & $\begin{array}{l}\text { Sample } \\
\text { Date }\end{array}$ & Filter & Value & $\begin{array}{c}\text { Analytical } \\
\text { Units }\end{array}$ & $\begin{array}{c}\text { Lab } \\
\text { Qualifier }\end{array}$ & $\begin{array}{c}\text { Counting } \\
\text { Error }\end{array}$ & $\begin{array}{l}\text { Total } \\
\text { Analysis } \\
\text { Error }\end{array}$ \\
\hline \multicolumn{8}{|c|}{$\begin{array}{c}\text { Well 299-E33-334 } \\
279 \mathrm{ft} \text { bgs } \\
\end{array}$} \\
\hline Aluminum & $1 / 5 / 00$ & $\mathrm{Y}$ & 19.7 & $\mu \mathrm{g} / \mathrm{L}$ & $\mathrm{U}$ & & \\
\hline Iron & $1 / 5 / 00$ & $Y$ & 49.2 & $\mu \mathrm{g} / \mathrm{L}$ & B & & \\
\hline Magnesium & $1 / 5 / 00$ & $\mathrm{Y}$ & 10300 & $\mu \mathrm{g} / \mathrm{L}$ & & & \\
\hline Potassium & $1 / 5 / 00$ & $\mathrm{Y}$ & 5550 & $\mu g / L$ & & & \\
\hline Nickel & $1 / 5 / 00$ & $\mathrm{Y}$ & 12.8 & $\mu g / L$ & $\mathrm{U}$ & & \\
\hline Manganese & $1 / 5 / 00$ & $Y$ & 11.3 & $\mu \mathrm{g} / \mathrm{L}$ & $\mathrm{B}$ & & \\
\hline Silver & $1 / 5 / 00$ & $\mathrm{Y}$ & 5.5 & $\mu \mathrm{g} / \mathrm{L}$ & $\mathbf{U}$ & & \\
\hline Strontium (elemental) & $1 / 5 / 00$ & $\mathrm{Y}$ & 169 & $\mu \mathrm{g} / \mathrm{L}$ & & & \\
\hline Barium & $1 / 5 / 00$ & $\mathrm{Y}$ & 61.1 & $\mu \mathrm{g} / \mathrm{L}$ & $\mathrm{B}$ & & \\
\hline Antimony & $1 / 5 / 00$ & $\mathrm{Y}$ & 40.9 & $\mu \mathrm{g} / \mathrm{L}$ & $U$ & & \\
\hline Calcium & $1 / 5 / 00$ & $\mathrm{Y}$ & 34200 & $\mu \mathrm{g} / \mathrm{L}$ & & & \\
\hline Zinc & $1 / 5 / 00$ & $\mathrm{Y}$ & 183 & $\mu \mathrm{g} / \mathrm{L}$ & & & \\
\hline Vanadium & $1 / 5 / 00$ & $\mathrm{Y}$ & 22.3 & $\mu g / L$ & B & & \\
\hline Copper & $1 / 5 / 00$ & $\mathrm{Y}$ & 6.4 & $\mu \mathrm{g} / \mathrm{L}$ & $\mathrm{U}$ & & \\
\hline Cobalt & $1 / 5 / 00$ & $\mathrm{Y}$ & 2.8 & $\mu \mathrm{g} / \mathrm{L}$ & $\mathrm{U}$ & & \\
\hline Chromium & $1 / 5 / 00$ & $\mathbf{Y}$ & 3 & $\mu \mathrm{g} / \mathrm{L}$ & $\mathrm{U}$ & & \\
\hline Cadmium & $1 / 5 / 00$ & $\mathbf{Y}$ & 3.3 & $\mu \mathrm{g} / \mathrm{L}$ & $\mathrm{U}$ & & \\
\hline Beryllium & $1 / 5 / 00$ & $\mathrm{Y}$ & 0.5 & $\mu \mathrm{g} / \mathrm{L}$ & $\mathrm{U}$ & & \\
\hline Sodium & $1 / 5 / 00$ & $\mathrm{Y}$ & 21300 & $\mu \mathrm{g} / \mathrm{L}$ & & & \\
\hline Sulfate & $1 / 5 / 00$ & $\mathrm{~N}$ & 41.5 & $\mathrm{mg} / \mathrm{L}$ & $\mathrm{D}$ & & \\
\hline Chloride & $1 / 5 / 00$ & $\mathrm{~N}$ & 14.7 & $\mathrm{mg} / \mathrm{L}$ & $\mathrm{D}$ & & \\
\hline Cyanide & $1 / 5 / 00$ & $\mathbf{N}$ & 1.6 & $\mu \mathrm{g} / \mathrm{L}$ & $\mathrm{U}$ & & \\
\hline Fluoride & $1 / 5 / 00$ & $\mathrm{~N}$ & 0.36 & $\mathrm{mg} / \mathrm{L}$ & & & \\
\hline Nitrogen in Nitrite & $1 / 5 / 00$ & $\mathrm{~N}$ & 0.0074 & $\mathrm{mg} / \mathrm{L}$ & $\mathrm{U}$ & & \\
\hline pH Measurement & $1 / 5 / 00$ & $\mathrm{~N}$ & 8 & $\mathrm{pH}$ & & & \\
\hline Temperature & $1 / 5 / 00$ & $\mathrm{~N}$ & 16 & Deg C & & & \\
\hline Turbidity & $1 / 5 / 00$ & $\mathrm{~N}$ & 0.7 & NTU & & & \\
\hline pH Measurement & $1 / 5 / 00$ & $\mathrm{~N}$ & 8.1 & $\mathrm{pH}$ & & & \\
\hline Nitrogen in Nitrate & $1 / 5 / 00$ & $\mathrm{~N}$ & 3.4 & $\mathrm{mg} / \mathrm{L}$ & $\mathrm{D}$ & & \\
\hline Dissolved Oxygen & $1 / 5 / 00$ & $\mathrm{~N}$ & 6.51 & $\mathrm{mg} / \mathrm{L}$ & & & \\
\hline Specific Conductance & $1 / 5 / 00$ & $\mathrm{~N}$ & 369 & $\mu \mathrm{S} / \mathrm{cm}$ & & & \\
\hline Tritium & $1 / 5 / 00$ & $\mathrm{~N}$ & 7140 & $\mathrm{pCi} / \mathrm{L}$ & & 330 & 600 \\
\hline Cesium-137 & $1 / 5 / 00$ & $\mathrm{~N}$ & -1.21 & $\mathrm{pCi} / \mathrm{L}$ & $\mathbf{U}$ & 4.3 & 4.3 \\
\hline Cobalt- 60 & $1 / 5 / 00$ & $\mathrm{~N}$ & 1.27 & $\mathrm{pCi} / \mathrm{L}$ & $\mathrm{U}$ & 3.9 & 3.9 \\
\hline
\end{tabular}


Table D.1. (contd)

\begin{tabular}{|c|c|c|c|c|c|c|c|}
\hline Constituent & $\begin{array}{c}\text { Sample } \\
\text { Date }\end{array}$ & Filter & Value & $\begin{array}{l}\text { Analytical } \\
\text { Units }\end{array}$ & $\begin{array}{c}\text { Lab } \\
\text { Qualifier }\end{array}$ & $\begin{array}{l}\text { Counting } \\
\text { Error }\end{array}$ & $\begin{array}{c}\text { Total } \\
\text { Analysis } \\
\text { Error }\end{array}$ \\
\hline Strontium-90 & $1 / 5 / 00$ & $\mathrm{~N}$ & -0.00897 & $\mathrm{pCi} / \mathrm{L}$ & $\mathrm{U}$ & 0.23 & 0.23 \\
\hline Gross beta & $1 / 5 / 00$ & $\mathrm{~N}$ & 15 & $\mathrm{pCi} / \mathrm{L}$ & & 2.3 & 3 \\
\hline Beryllium-7 & $1 / 5 / 00$ & $\mathrm{~N}$ & 9.16 & $\mathrm{pCi} / \mathrm{L}$ & $\mathbf{U}$ & 63 & 63 \\
\hline Cesium-134 & $1 / 5 / 00$ & $\mathrm{~N}$ & -3.12 & $\mathrm{pCi} / \mathrm{L}$ & $\mathbf{U}$ & 4.6 & 4.6 \\
\hline Antimony-125 & $1 / 5 / 00$ & $\mathrm{~N}$ & -1.47 & $\mathrm{pCi} / \mathrm{L}$ & $\mathrm{U}$ & 12 & 12 \\
\hline Europium-152 & $1 / 5 / 00$ & $\mathbf{N}$ & -4.94 & $\mathrm{pCi} / \mathrm{L}$ & $\mathrm{U}$ & 10 & 10 \\
\hline Europium-155 & $1 / 5 / 00$ & $\mathrm{~N}$ & -3.41 & $\mathrm{pCi} / \mathrm{L}$ & $\mathbf{U}$ & 11 & 11 \\
\hline Uranium & $1 / 5 / 00$ & $\mathrm{~N}$ & 2.75 & $\mu \mathrm{g} / \mathrm{L}$ & & & 0.44 \\
\hline Europium-154 & $1 / 5 / 00$ & $\mathrm{~N}$ & -6.45 & $\mathrm{pCi} / \mathrm{L}$ & $\mathbf{U}$ & 14 & 14 \\
\hline Technetium-99 & $1 / 5 / 00$ & $\mathrm{~N}$ & 58.9 & $\mathrm{pCi} / \mathrm{L}$ & & 2.4 & 15 \\
\hline Ruthenium-106 & $1 / 5 / 00$ & $\mathbf{N}$ & 3.22 & $\mathrm{pCi} / \mathrm{L}$ & $\mathrm{U}$ & 37 & 37 \\
\hline Potassium-40 & $1 / 5 / 00$ & $\mathbf{N}$ & -3.12 & $\mathrm{pCi} / \mathrm{L}$ & $\mathrm{U}$ & 68 & 68 \\
\hline Gross alpha & $1 / 5 / 00$ & $\mathbf{N}$ & 0.922 & $\mathrm{pCi} / \mathrm{L}$ & $\mathrm{U}$ & 1.1 & 1.1 \\
\hline \multicolumn{8}{|c|}{$\begin{array}{c}\text { Well 299-E33-335 } \\
272 \mathrm{ft} \text { bgs }\end{array}$} \\
\hline Specific Conductance & $2 / 10 / 00$ & $\mathrm{~N}$ & 370 & $\mu \mathrm{S} / \mathrm{cm}$ & & & \\
\hline pH Measurement & $2 / 10 / 00$ & $\mathbf{N}$ & 8.18 & $\mathrm{pH}$ & & & \\
\hline Turbidity & $2 / 10 / 00$ & $\mathbf{N}$ & 0.92 & NTU & & & \\
\hline Temperature & $2 / 10 / 00$ & $\mathbf{N}$ & 16.1 & Deg C & & & \\
\hline Tritium & $2 / 10 / 00$ & $\mathbf{N}$ & 6880 & $\mathrm{pCi} / \mathrm{L}$ & & 340 & 620 \\
\hline Cesium-137 & $2 / 10 / 00$ & $\mathrm{~N}$ & 0.261 & $\mathrm{pCi} / \mathrm{L}$ & $\mathbf{U}$ & 3.2 & 3.2 \\
\hline Strontium-90 & $2 / 10 / 00$ & $\mathbf{N}$ & -0.0384 & $\mathrm{pCi} / \mathrm{L}$ & $\mathbf{U}$ & 0.27 & 0.27 \\
\hline Gross alpha & $2 / 10 / 00$ & $\mathrm{~N}$ & 1.81 & $\mathrm{pCi} / \mathrm{L}$ & $\mathbf{U}$ & 1.4 & 1.5 \\
\hline Beryllium-7 & $2 / 10 / 00$ & $\mathbf{N}$ & -15.2 & $\mathrm{pCi} / \mathrm{L}$ & $\mathrm{U}$ & 41 & 41 \\
\hline Cesium-134 & $2 / 10 / 00$ & $\mathbf{N}$ & -1.56 & $\mathrm{pCi} / \mathrm{L}$ & $\mathrm{U}$ & 3.7 & 3.7 \\
\hline Antimony-125 & $2 / 10 / 00$ & $\mathbf{N}$ & 2.25 & $\mathrm{pCi} / \mathrm{L}$ & $\mathbf{U}$ & 8.8 & 8.8 \\
\hline Europium-152 & $2 / 10 / 00$ & $\mathbf{N}$ & 0.463 & $\mathrm{pCi} / \mathrm{L}$ & U & 9.5 & 9.5 \\
\hline Uranium & $2 / 10 / 00$ & $\mathbf{N}$ & 2.74 & $\mu \mathrm{g} / \mathrm{L}$ & & & 0.65 \\
\hline Europium-154 & $2 / 10 / 00$ & $\mathbf{N}$ & 4.27 & $\mathrm{pCi} / \mathrm{L}$ & $\mathrm{U}$ & 9.2 & 9.2 \\
\hline Europium-155 & $2 / 10 / 00$ & $\mathbf{N}$ & -4.62 & $\mathrm{pCi} / \mathrm{L}$ & $\mathrm{U}$ & 6.9 & 6.9 \\
\hline Technetium-99 & $2 / 10 / 00$ & $\mathbf{N}$ & 59.2 & $\mathrm{pCi} / \mathrm{L}$ & & 2.4 & 15 \\
\hline Potassium -40 & $2 / 10 / 00$ & $\mathbf{N}$ & 8.97 & $\mathrm{pCi} / \mathrm{L}$ & $\mathrm{U}$ & 53 & 53 \\
\hline Gross beta & $2 / 10 / 00$ & $\mathbf{N}$ & 18.3 & $\mathrm{pCi} / \mathrm{L}$ & & 2.4 & 3.4 \\
\hline Cobalt-60 & $2 / 10 / 00$ & $\mathbf{N}$ & 0.546 & $\mathrm{pCi} / \mathrm{L}$ & $\mathrm{U}$ & 3.4 & 3.4 \\
\hline Ruthenium-106 & $2 / 10 / 00$ & $\mathrm{~N}$ & -13.7 & $\mathrm{pCi} / \mathrm{L}$ & $\mathrm{U}$ & 32 & 32 \\
\hline Specific Conductance & $3 / 2 / 00$ & $\mathrm{~N}$ & 368 & $\mu \mathrm{S} / \mathrm{cm}$ & C & & \\
\hline Total organic halides & $3 / 2 / 00$ & $\mathrm{~N}$ & 4.3 & $\mu \mathrm{g} / \mathrm{L}$ & $\mathrm{U}$ & & \\
\hline
\end{tabular}


Table D.1. (contd)

\begin{tabular}{|c|c|c|c|c|c|c|c|}
\hline Constituent & $\begin{array}{c}\text { Sample } \\
\text { Date }\end{array}$ & Filter & Value & $\begin{array}{l}\text { Analytical } \\
\text { Units }\end{array}$ & $\begin{array}{c}\text { Lab } \\
\text { Qualifier }\end{array}$ & $\begin{array}{c}\text { Counting } \\
\text { Error }\end{array}$ & $\begin{array}{c}\text { Total } \\
\text { Analysis } \\
\text { Error }\end{array}$ \\
\hline Specific Conductance & $3 / 2 / 00$ & $\mathbf{N}$ & 382 & $\mu \mathrm{S} / \mathrm{cm}$ & & & \\
\hline Temperature & $3 / 2 / 00$ & $\mathrm{~N}$ & 17.5 & Deg C & & & \\
\hline pH Measurement & $3 / 2 / 00$ & $\mathrm{~N}$ & 8.03 & $\mathrm{pH}$ & & & \\
\hline Total organic carbon & $3 / 2 / 00$ & $\mathrm{~N}$ & 0.6 & $\mathrm{mg} / \mathrm{L}$ & B & & \\
\hline Total organic halides & $3 / 2 / 00$ & $\mathrm{~N}$ & 4.3 & $\mu g / L$ & $\mathbf{U}$ & & \\
\hline Specific Conductance & $3 / 2 / 00$ & $\mathrm{~N}$ & 382 & $\mu \mathrm{S} / \mathrm{cm}$ & & & \\
\hline Temperature & $3 / 2 / 00$ & $\mathrm{~N}$ & 17.5 & Deg C & & & \\
\hline Total organic carbon & $3 / 2 / 00$ & $\mathrm{~N}$ & 0.69 & $\mathrm{mg} / \mathrm{L}$ & $\mathrm{B}$ & & \\
\hline pH Measurement & $3 / 2 / 00$ & $\mathrm{~N}$ & 8.03 & $\mathrm{pH}$ & & & \\
\hline Total organic halides & $3 / 2 / 00$ & $\mathrm{~N}$ & 4.3 & $\mu \mathrm{g} / \mathrm{L}$ & $\mathbf{U}$ & & \\
\hline Specific Conductance & $3 / 2 / 00$ & $\mathrm{~N}$ & 382 & $\mu \mathrm{S} / \mathrm{cm}$ & & & \\
\hline pH Measurement & $3 / 2 / 00$ & $\mathrm{~N}$ & 8.03 & $\mathrm{pH}$ & & & \\
\hline Total organic carbon & $3 / 2 / 00$ & $N$ & 0.59 & $\mathrm{mg} / \mathrm{L}$ & B & & \\
\hline Temperature & $3 / 2 / 00$ & $\mathbf{N}$ & 17.5 & Deg C & & & \\
\hline Total organic halides & $3 / 2 / 00$ & $\mathbf{N}$ & 4.3 & $\mu \mathrm{g} / \mathrm{L}$ & $\mathbf{U}$ & & \\
\hline Specific Conductance & $3 / 2 / 00$ & $\mathrm{~N}$ & 382 & $\mu \mathrm{S} / \mathrm{cm}$ & & & \\
\hline Temperature & $3 / 2 / 00$ & $\mathrm{~N}$ & 17.5 & $\operatorname{Deg} C$ & & & \\
\hline pH Measurement & $3 / 2 / 00$ & $\mathrm{~N}$ & 8.02 & $\mathrm{pH}$ & & & \\
\hline Turbidity & $3 / 2 / 00$ & $\mathrm{~N}$ & 2.8 & NTU & & & \\
\hline Total organic carbon & $3 / 2 / 00$ & $\mathrm{~N}$ & 0.74 & $\mathrm{mg} / \mathrm{L}$ & $\mathrm{B}$ & & \\
\hline Aluminum & $3 / 2 / 00$ & $Y$ & 19.7 & $\mu \mathrm{g} / \mathrm{L}$ & $\mathbf{U}$ & & \\
\hline Iron & $3 / 2 / 00$ & $\mathbf{Y}$ & 65.8 & $\mu \mathrm{g} / \mathrm{L}$ & B & & \\
\hline Magnesium & $3 / 2 / 00$ & $\bar{Y}$ & 11100 & $\mu \mathrm{g} / \mathrm{L}$ & & & \\
\hline Manganese & $3 / 2 / 00$ & $Y$ & 17.3 & $\mu \mathrm{g} / \mathrm{L}$ & & & \\
\hline Sodium & $3 / 2 / 00$ & $Y$ & 19600 & $\mu \mathrm{g} / \mathrm{L}$ & & & \\
\hline Antimony & $3 / 2 / 00$ & $\mathrm{Y}$ & 40.9 & $\mu \mathrm{g} / \mathrm{L}$ & $\mathbf{U}$ & & \\
\hline Beryllium & $3 / 2 / 00$ & $Y$ & 0.5 & $\mu \mathrm{g} / \mathrm{L}$ & $\mathbf{U}$ & & \\
\hline Chromium & $3 / 2 / 00$ & $Y$ & 5.2 & $\mu \mathrm{g} / \mathrm{L}$ & B & & \\
\hline Copper & $3 / 2 / 00$ & $Y$ & 6.4 & $\mu \mathrm{g} / \mathrm{L}$ & $\mathbf{U}$ & & \\
\hline Cobalt & $3 / 2 / 00$ & $Y$ & 2.8 & $\mu \mathrm{g} / \mathrm{L}$ & $\mathbf{U}$ & & \\
\hline Calcium & $3 / 2 / 00$ & $\bar{Y}$ & 37200 & $\mu \mathrm{g} / \mathrm{L}$ & & & \\
\hline Zinc & $3 / 2 / 00$ & $Y$ & 13.8 & $\mu \mathrm{g} / \mathrm{L}$ & B & & \\
\hline Vanadium & $3 / 2 / 00$ & $\mathrm{Y}$ & 15.8 & $\mu \mathrm{g} / \mathrm{L}$ & B & & \\
\hline Cadmium & $3 / 2 / 00$ & $\mathrm{Y}$ & 3.3 & $\mu \mathrm{g} / \mathrm{L}$ & $\mathbf{U}$ & & \\
\hline Barium & $3 / 2 / 00$ & $\mathrm{Y}$ & 47.2 & $\mu \mathrm{g} / \mathrm{L}$ & B & & \\
\hline
\end{tabular}


Table D.1. (contd)

\begin{tabular}{|l|r|r|r|l|c|c|c||}
\hline \multicolumn{1}{|c|}{ Constituent } & $\begin{array}{c}\text { Sample } \\
\text { Date }\end{array}$ & Filter & Value & $\begin{array}{c}\text { Analytical } \\
\text { Units }\end{array}$ & $\begin{array}{c}\text { Lab } \\
\text { Qualifier }\end{array}$ & $\begin{array}{c}\text { Counting } \\
\text { Error }\end{array}$ & $\begin{array}{c}\text { Total } \\
\text { Analysis } \\
\text { Error }\end{array}$ \\
\hline Strontium (elemental) & $3 / 2 / 00$ & $\mathrm{Y}$ & 197 & $\mu \mathrm{g} / \mathrm{L}$ & & & \\
\hline Silver & $3 / 2 / 00$ & $\mathrm{Y}$ & 5.5 & $\mu \mathrm{g} / \mathrm{L}$ & $\mathrm{U}$ & & \\
\hline Potassium & $3 / 2 / 00$ & $\mathrm{Y}$ & 5310 & $\mu \mathrm{g} / \mathrm{L}$ & & & \\
\hline Nickel & $3 / 2 / 00$ & $\mathrm{Y}$ & 12.8 & $\mu \mathrm{g} / \mathrm{L}$ & $\mathrm{U}$ & & \\
\hline Sulfate & $3 / 2 / 00$ & $\mathrm{~N}$ & 43.5 & $\mathrm{mg} / \mathrm{L}$ & $\mathrm{D}$ & & \\
\hline Chloride & $3 / 2 / 00$ & $\mathrm{~N}$ & 16.3 & $\mathrm{mg} / \mathrm{L}$ & $\mathrm{D}$ & & \\
\hline Fluoride & $3 / 2 / 00$ & $\mathrm{~N}$ & 0.37 & $\mathrm{mg} / \mathrm{L}$ & & & \\
\hline Alkalinity & $3 / 2 / 00$ & $\mathrm{~N}$ & 122 & $\mathrm{mg} / \mathrm{L}$ & & & \\
\hline Total dissolved solids & $3 / 2 / 00$ & $\mathrm{~N}$ & 241 & $\mathrm{mg} / \mathrm{L}$ & & & \\
\hline Nitrogen in Nitrate & $3 / 2 / 00$ & $\mathrm{~N}$ & 3.4 & $\mathrm{mg} / \mathrm{L}$ & $\mathrm{D}$ & & \\
\hline Nitrogen in Nitrite & $3 / 2 / 00$ & $\mathrm{~N}$ & 0.0074 & $\mathrm{mg} / \mathrm{L}$ & $\mathrm{U}$ & & \\
\hline Cyanide & $3 / 2 / 00$ & $\mathrm{~N}$ & 1.6 & $\mu \mathrm{g} / \mathrm{L}$ & $\mathrm{U}$ & & \\
\hline \hline
\end{tabular}




\section{Distribution}

No. of

Copies

OFFSITE

R. Jim

Confederated Tribes and Bands of the

Yakama Nation

Environmental Restoration/Waste

Management

2808 Main Street

Union Gap, WA 98903

L. Seelatsee

Wanapum Band

Grant County P.U.D.

30 "C" Street S.W.

P. O. Box 878

Ephrata, WA 98823

P. Sobotta

Nez Perce Tribe

Environmental Restoration/Waste

Management

P.O. Box 365

Lapwai, ID 83540-0365

J. R. Wilkerson

Confederated Tribes of the Umatilla Indian

Reservation

Environmental Planning/Rights Protection

P. O. Box 638

Pendleton, OR 97801
No. of

Copies

ONSITE

3 DOE Richland Operations Office

M.J. Furman (2)

A5-13

R. M. Yasek

H6-60

3 CH2M Hill Group
A. J. Knepp (3)
$\mathrm{H} 0-22$

2 CH2M Hill Hanford, Inc.

$\begin{array}{ll}\text { J. V. Borghese } & \text { H9-03 } \\ \text { D. C. Weekes } & \text { H9-02 }\end{array}$

U.S. Environmental Protection Agency

D. A. Fāulk

B5-01

3 Washington State Department of Ecology

D. Goswami B5-18

A. D. Huckaby B5-18

$\begin{array}{ll}\text { S. Leja } & \text { B5-18 }\end{array}$

Pacific Northwest National Laboratory

D. G. Horton (3) K6-81

S. P. Luttrell K6-96

W. J. Martin K6-81

S. M. Narbutovskih (3) K6-96

B. A. Williams $\quad \mathrm{K} 6-81$

Information Release Office (7) K1-06

Distr.1 